\title{
CARACTÉRISTIQUES SCALIMÉTRIQUES DES PRINCIPALES ESPĖCES DE POISSONS D'EAU DOUCE DE FRANCE
}

\author{
J.L. BAGLINIERE, H. LE LOUARN \\ Station de Physiologie et d'Ecologie des Poissons, \\ I.N.R.A., 65, route de St-Brieuc - 35042 RENNES Cedex, France.
}

Recu le 4 août 1986

Accepté le 21 août 1987

Received 4 August, 1986

Accepted 21 August, 1987

\section{RÉSUME}

Ce document permet une reconnaissance de 41 espèces de poissons d'eau douce les plus communes rencontrées en France à partir des caractéristiques de leurs écailles (forme et structure). L'utilisation de ces écailles pour la détermination de l'âge est analysée.

MOTS-CLES : poisson, eau douce, écaille, détermination de l'âge.

\section{SCALES CHARACTERISTICS OF THE MAIN FRESHWATER FISH SPECIES IN FRANCE}

\section{ABSTRACT}

This work allows a recognition of 41 the more common freshwater fish species in France from the scales characteristics (form and structure). The use of scales for the age determination is analysed.

KEY-WORDS : fish, freshwater, scale, age determination

\section{INTRODUCTION}

Les écailles, au contraire des formations osseuses rencontrées chez les Sélaciens, sont des phanères exclusivement dermiques. Vestiges des cuirasses osseuses des poissons les plus archaïques, elles sont propres aux Ostéichthyens (BERTIN in GRASSE, 1958; MEUNIER, 1983; BURDAK, 1986). Dans cette classe, l'évolution de l'écaille est inverse de celle de l'endosquelette : plus celui-ci se renforce et plus l'écaillure se restreint et s'amincit (BERTIN in GRASSE, 1958). La formation des écailles élasmoïdes entièrement osseuses des Téléostéens, groupe le plus tardif des Ostéichthyens, a entraîné la mise en place d'un revêtement écaiłleux léger et solide qui gêne très peu les mouvements du poisson. Ceci a permis un développement des adaptations les plus variées des caractères défensifs, cryptiques et hydrodynamiques liés à ce revêtement (MEUNIER, 1983; BURDAK. 1986).

Les premières études fondamentales sur la morphologie et la classification des écailles de Poissons et Agnathes furent effectuées par AGASSIZ (1833) et BAUDELOT (1873) in BURDAK (1986) Plus récemment, un tel travail a été repris par BERTIN in GRASSE (1958). L'importance des écailles dans la systématique des poissons a permis à MAITLAND (1972) de mettre au point une clé d'identification des familles à partir des écailles. Devant l'ampleur prise par les travaux en biologie et dynamique de population des poissons d'eau douce en France, il est apparu intéressant de synthétiser les caractéristiques scalimétriques des espèces dulçaquicoles les plus communes. Ainsi le travail présenté recouvre deux aspects essentiels :

- la description des écailles au stade adulte à partir d'illustrations.

- l'analyse des avantages et des inconvénients de la méthode scalimétrique pour la détermination de

l'âge et la mesure de la croissance, en général et pour chaque espèce en particulier.

Ce document veut constituer un ouvrage pratique facilement utilisable par les gestionnaires. II peut en outre compléter l'Atlas de référence des espèces dulçaquicoles réalisé au niveau national par le Secrétariat Faune et Flore du Muséum d'Histoire Naturelle et coordonné par ALLARDI (com. pers.). 


\title{
2. ESPĖCES ÉTUDIÉES
}

Les espèces étudiées appartiennent aux ordres et familles suivants (BERRA, 1981):

\author{
Ordre des Clupéiformes \\ Famille des Clupéidés \\ Ordre des Salmoniformes \\ Famille des Salmonidés \\ Famille des Esocidés \\ Ordre des Athériniformes \\ Famille des Poécilidés \\ Ordre des Cypriniformes \\ Famille des Cyprinidés \\ Ordre des Perciformes \\ Famille des Percidés \\ Famille des Centrarchidés \\ Ordre des Mugiliformes \\ Famille des Mugilidés
}

Un certain nombre d'espèces n'a pas été pris en compte en raison:

- d'une absence d'écailles, BURDAK, 1986 : lamproie marine Petromyzon marinus Linné; lamproie fluviatile Lampetra fluviatilis Linné; lamproie de Planer Lampetra planeri Bloch; l'esturgeon Acipenser sturio Linné dont l'âge est déterminé à partir des sections de rayons de nageoire (JEARLD, 1983; TROUVERY et WILLIOT, 1984), l'épinoche Gasterosteus aculeatus Linné et l'épinochette Pungitius pungitius Linné pour lesquelles l'âge est déterminé à partir des otolithes JONES et HYNES. 1950; ALLEN et WOOTTON, 1982); le poisson chat /ctalurus melas Rafinesque dont l'âge est déterminé à partir des sections de rayons de nageoires (BOET, 1981; JEARLD, 1983); le silure glane Silurus glanis Linné ;

- d'une distribution géographique très restreinte : I'apron Zingel asper Linné ; le blageon Leuciscus souffia Risso;

- de l'utilisation d'autres structures, telles que les otolithes, pour la détermination de l'âge et l'analyse de la croissance : la lotte Lotta lotta Linné comme tous les Gadidés (JEARLD, 1983); l'atherine Atherina mochon Cuvier (JEARLD, 1983); le chabot Cottus gobio Linné (CRISP, 1963; PETROSKY et WATERS, 1975); pour cette espèce les vertèbres sont également utilisées (KIRKA, 1969); la loche d'étang Misgurnus fossilis Linné; la loche de rivière Cobitis taenia Linné; la loche franche Noemacheilus barbatulus Linné (SMILY, 1955; MILLS et al., 1983); I'anguille Anguilla anguilla Linné (JEARLD, 1983); le flet Platichthys flesus Linné (DENIEL, 1981 ; JEARLD, 1983); pour ces deux dernières espèces l'écaille est cependant présentée à titre indicatif (figure 1a et b).

\section{PRÉLEVEMENT, PRÉPARATION ET DESCRIPTION D'UNE ÉCAILle}

\section{1. - Prélèvement et préparation}

Le prélèvement a été effectué dans des zones spécifiques (CASSELMANN, 1967 ; figure 2), sauf pour les Centrarchidés et les Percidés (JEARLD, 1983). Ces zones ne correspondent pas toujours à celles de la mise en place des premières écailles (SIRE, 1981). Deux types de montage ont été utilisés :

- après nettoyage dans une solution de peroxyde de sodium ou de potasse à 5 p. 100, montage à sec entre deux lames ou bien entre lame et lamelle:

- impression sur film plastique transparent d'un $\mathrm{mm}$ d'épaisseur sans nettoyage. Cette méthode préconisée depuis de nombreuses années (NESBIT, 1934) est, depuis peu, de plus en plus utilisée en France en particulier pour les Salmonidés (BAGLINIĖRE, 1985).

Trois à quatre écailles ont été prélevées sur plusieurs individus d'une mème espèce au stade adulte à un âge supérieur à un an. Seule l'écaille la mieux préparée et la plus lisible a été retenue pour l'étude.

\section{2. - Description générale}

L'observation a été faite à l'aide d'un lecteur de profil (X 10 à 100).

Caractéristiques des poissons les plus évolués, les écailles élasmoïdes sont des structures minces, transparentes et lamelleuses. Leur forme varie mais reste toujours plus ou moins ovalaire, avec un léger bombement sur la face supérieure. La surface peut être uniforme ou divisée en champs (figure 3). Dans ce dernier cas on peut distinguer un champ antérieur, deux champs latéraux et un 
champ postérieur qui est la partie visible de l'écaille en place dans son repli épidermique. Sur toute la surface, mais avec des aspects différents suivant le champ, on distingue deux types de relief superficiels ayant un rôle important dans la fonction hydrodynamique de l'écaille (MEUNIER, 1983, BURDAK, 1986)

- les crêtes circulaires ou circuli, plus ou moins concentriques autour du focus et disposées parallèlement au bord de l'écaille. Sur sa partie découverte (champ postérieur), ils sont toujours disposés suivant une direction générale perpendiculaire à celle de la friction de l'eau.

- les reliefs rayonnants se présentant sous formes de crêtes ou de sillons radiaires avec dans certains cas des sections transverses plus ou moins régulières (- radii).

Il existe deux variantes de l'écaille élasmoïde (BERTIN in GRASSE, 1958; BURDAK, 1986).

- les écailles cycloides. Les plus typiques sont celles du genre Salmo sans radii ni champs distincts:

- les écailles cténoïdes: des spinules (- ctenii) s'observent sur le bord postérieur de l'écaille ou peuvent recouvrir tout le champ postérieur. Elles sont caractéristiques de l'ordre des Perciformes et des Mugiliformes.

A part ces quelques différences, ces deux variantes de l'écaille élasmoïde sont tout à fait identiques, et en ontogenèse comme en phylogenèse, on passe de l'une à l'autre dans des circonstances bien déterminées (BURDAK, 1986). Ces deux variantes peuvent même être présentes en même temps chez certaines espèces (SIRE et MEUNIER, 1981). En l'état actuel des connaissances, la division des écailles élasmoides en deux types cycloïde et cténoide, basée seulement sur la présence ou l'absence de ctenii est artificielle et insuffisante pour une classification typologique des écailles de Téléostéens (MEUNIER, 1983; MEUNIER, 1984a)

\section{DÉtermination DE L'AGE ET DE LA CROISSANCE}

\section{1. - Généralités}

Si chaque espèce de poisson présente une taille moyenne caractéristique, la croissance individuelle n'atteint pas au cours de la vie une limite théorique. A un âge donné, la taille va donc varier considérablement en fonction des facteurs génétiques et des conditions de vie. DAHL (1909) a le premier noté que la croissance de l'écaille est reliée à celle du corps par une relation mathématique.

Pourtant, la croissance n'est pas continue puisque les poissons, vertébrés poikilothermes, ont un métabolisme modulé en grande partie par la température. Dans les régions à hivers marqués, les rythmes saisonniers de croissance se marquent dans le tissu osseux par des zones caractéristiques. II sera donc possible de donner l'âge du poisson. Différentes structures osseuses ont été utilisées dans ce but : écailles (HOFFBAUER, 1898, et ensuite de nombreux auteurs dont JARVI et MENZIES, 1936 ; CASSELMANN, 1974; MEUNIER et PASCAL, 1980; JEARLD, 1983); Opercules (LECREN, 1947); Rayons des nageoires (CUERRIER, 1951): Vertèbres (MARZOLF, 1955). Une revue en est faite par MENON (1950) et plus récemment par BOET (1981) et BOET et LE LOUARN (1985).

\section{2. - Scalimétrie : utilisation et limites}

La scalimétire fut la première technique utilisée (HOFFBAUER, 1898; LEA, 1910) et reste encore la plus fréquente en raison de la possibilité qu'elle offre de relâcher le poisson et des facilités de prélèvement, de préparation et de lecture (BAGLINIÉRE, 1985 ; BAGLINIĖRE et al., 1986).

La croissance de l'écaille n'est pas régulière sur toute sa surface. La partie antérieure, incluse plus profondément dans le derme, montre en génér al un développement plus important. Le développement de la partie postérieure plus externe est au contraire réduit. En fait, la partie la plus lisible de l'écaille est constituée par les champs latéraux (CASSELMANN, 1967) sauf cas particuliers.

Une croissance rapide se traduit par des circuli espacés et une zone claire. Lorsqu'elle se ralentit, les circuli sont rapprochés et forment un anneau plus foncé appelé annulus (JEARLD, 1983) dont la limite théorique est le premier circulus discontinu présent avant la nouvelle zone annuelle (Anonyme, 1984). Le nombre de ces annuli ou hivers-écaille donne l'âge du poisson.

La lecture des écailles est la plus exploitable si le prélèvement est effectué juste avant ou pendant la phase d'arrêt de croissance. La période la plus critique est le printemps car la date de la reprise de croissance n'est ni fixe ni simultanée pour les individus d'une même espèce, ou de différentes espèces. (Cas du brochet : MONGEAU, 1960; cas des salmonidés : BAGLINIËRE et MAISSE, données non publiées). La présence d'une reprise de croissance indépendamment du nombre de circuli formés est conventionnellement notée plus $(+)$.

Certaines limites doivent être fixées aux interprétations des lectures d'écailles

- dans les milieux à saisons peu marquées, la croissance est pratiquement continue et l'arrêt hivernal se lit très mal : cas de la truite commune dans le bassin de la Nivelle (CHANCEREL, données non publiées). 
- dans les milieux oủ la croissance est faible, l'anneau hivernal peut ne plus se former sur les écailles des individus âgés d'au moins cinq ans (HESTHAGEN, 1985). De même, dès l'apparition de la maturation sexuelle. la croissance se ralentit et l'écaille peut devenir difficilement interprétable (cas de la Truite: JONSSON (1976)).

- tout choc physiologique va se marquer sur la vitesse de craissance pouvant entraîner sur l'écaille le resserrement de quelques circuli appelé "check" ou faux anneau (BILTON et ROBINS, 1971 a et b) : stress de captures (OTTAWAY et SIMKISS, 1977), variations quantitatives de l'alimentation (BILTON et ROBINS, $1971 \mathrm{c}$ ), changements de milieux (BILTON, 1974). maladies et parasites (VAN OOSTEN, 1957), variations climatiques non saisonnières (HOFSTEDE, 1974). Pour distinguer ces faux anneaux, certaines précautions sont à prendre (CHUGUNOVA, 1963). Chez certaines espèces (Brochet, MONGEAU, 1960; Saumon atlantique. Anonyme, 1984 ; Rotengle, MANN et STEINMETZ, 1985) des normes ont été établies pour les différencier des véritables resserrements hivernaux.

- Une blessure entrainera la formation d'écailles régénérées où les ostéoblastes se disposeront au départ en désordre avant de reprendre leur régularité concentrique. Chez la plupart des espèces de Cyprinidés (LE LOUARN, données non publiées) les zones à écailles régénérées sont repérables par une disposition plus ou moins irrégulière des écailles.

- Signalons enfin l'existence d'un rythme circadien endogène de croissance de l'écaille (OTTAWAY, 1978) qui peut avoir une influence sur l'apparition des arrêts de croissance hivernaux.

\section{DESCRIPTION DES ÉCAILLES DES DIFFÉRENTES ESPĖCES}

La description générale de la forme et de l'ornementation superficielle de l'écaille est faite au niveau de la famille ou de la sous-famille (voire de l'espèce). Puis pour chacune des espèces, la structure des anneaux hivernaux est analysée et illustrée. Pour chaque illustration, le poisson est caractérisé par son origine géographique, sa date de capture, sa longueur (LT = Longueur Totale, LF - Longueur Fourche) et par son âge quand cela est possible.

\section{FAMILLE DES CLUPÉIDÉS GENRE ALOSA}

Elle regroupe les espèces d'Aloses présentes en France:

- la grande alose : Alosa alosa Linné,

- et l'alose feinte : Alosa fallax Lacépède, dont la majorité du cycle biologique se déroule en mer, la reproduction ayant lieu en eau douce.

Leurs écailles sont tout à fait typiques de celles des clupéidés (CASSOU-LEINS et CASSOULEINS, 1981). Leur forme est polygonale, présentant un léger dôme dans son champ antérieur (figure 4 a). Cette forme peut se modifier par suite d'une érosion des écailles lors du retour en rivière en liaison avec la maturation sexuelle (CASSOU-LEINS et CASSOU-LEINS, 1981 ; DOUCHEMENT, 1981 ; BOISNEAU-MENNESSON et al., 1986): de polygonale l'écaille devient plus ovale, le dôme du champ antérieur disparaissant. Le focus est excentré vers le champ postér ieur qui est de fait réduit (30 à $40 \%$ de la dimension de l'écaille) (CASSOU-LEINS et CASSOU-LEINS, 1981).

Trois grands types d'ornementations sont observés (DOUCHEMENT, 1981):

- des sillons transverses, fortement marqués, présents dans tous les champs. Ils recoupent les deux autres types d'ornementations en passant d'un champ latéral à l'autre:

- une striation très fine et serrée. Ces circuli sont déposés de manière relativement transverse et plus concentrique vers le bord de l'écaille. Cette striation est absente du champ postérieur:

- des anneaux concentriques présents uniquement dans les champs latéraux et antérieur (figure 4 b). Ils épousent le contour de l'écaille et définissent des limites d'arrêt de croissance ou anneaux d'âge. Ces lignes sont souvent claires, correspondant à une coupure ou bien à un changement d'épaisseur des stries fines (figure 4c). Chez ces espèces, les phases juvénile en eau douce et adulte en mer ne peuvent ètre distinguées sur les écailles. La présence d'une ligne concentrique claire épaisse au niveau de laquelle la distribution est totalement désorganisée définit un anneau de ponte. L'apparition d'un tel phénomène est peu fréquent compte tenu du très faible tauxde survie des géniteurs après la première fraie (BOISNEAU-MENNESSON et al., 1986). Enfin, il existe de f́aux anneaux qui peuvent être souvent distingués des annuli par une présence uniquement dans les champs latéraux. Un anneau dit surnuméraire peut apparaître après le premier arrêt de croissance mais sa signification biologique n'est pas connue : retour en eau douce et court séjour après la descente en mer (DOUCHEMENT, 1981) ou bien deuxième annulus en rivière avant la dévalaison (BOISNEAU-MENNESSON et al., 1986). Le phénomène de régénération du centre de l'écaille reste assez fréquent. Actuellement, si la scalimétrie reste une méthode pratique de détermination d'âge. elle reste à valider par l'utilisation d'autres techniques (BOISNEAU-MENNESSON et al., 1986). 


\title{
FAMILLE DES SALMONIDÉS
}

\author{
Sous-famille des Salmonidés, genre Salmo et Salvelinus
}

Cette sous-famille regroupe en France deux genres (Salmo et Salvelinus) et six espèces. Parmi ces dernières, trois ont été transplantées du continent Nord Américain dont la truite arc-en-ciel Salmo gairdneri Richardson, poisson à croissance rapide utilisé essentiellement en pisciculture commerciale. Ce poisson semble pouvoir se maintenir naturellement dans quelques cas : cours d'eau pyrénéens (DUMAS, 1976) et affluents du lac Léman (CHAMPIGNEULLE, données non publiées). Les deux autres espèces importées sont l'omble de fontaine (Salvelinus fontinalis) et le cristivomer (Salvelinus namaycush) respectivement introduites dans les cours d'eaux et les lacs de montagne en raison de préférences thermiques plus basses que le genre Salmo (PETERSON et al., 1979).

Dans cette sous-famille sont également présents des espèces ou écotypes de grands migrateurs en mer ou en lac (uniquement du genre Salmo)

L'écaille a une forme caractéristique circulaire à ovalaire nette ne permettant de distinguer que des champs latéraux. Cette forme peut varier avec l'âge et la taille des individus en liaison avec le taux de croissance ainsi qu'avec la maturation sexuelle chez les grands migrateurs (figure 5 a à j). Chez ces derniers, la forme semble suffisamment caractéristique au niveau d'un stock (rivière) pour qu'elle permette leur discrimination (Saumon atlantique (DE PONTUAL, 1986): Truite de mer (RICHARD. données non publiées)). Le focus reste relativement centré. Le champ postérieur est presque dépourvu de circuli. Ceux-ci sont déposés d'une manière concentrique et régulière autour du centre de l'écaille en épousant sa forme. Les anneaux hivernaux se présentent sous forme d'une bande de circuli resserrés quel que soit le milieu (rivière, lac ou mer) (figure $6 \mathrm{a}$ et b). Cependant, à nos latitudes, il semble que la truite de lac marque moins bien ces phases de ralentissement de croissance (figure $5 \mathrm{~g}$ ). Souvent, chez ces espèces, le premier anneau hivernal ne se marque que par. un changement net de l'espacement des circuli entre deux zones annuelles de l'écaille. Dès la première reproduction (elle peut avoir lieu chez les espèces grandes migratrices avant la descente en mer ou en lac (BAGLINIËRE, 1985); MAISSE et BAGLINIĖRE, données non publiées), il apparaît souvent une marque de fraie définie par une ligne continue concentrique avec recoupement de circuli (plus de parallélisme entre ceux déposés avant et après la fraie) et un important espacement inter-circuli après la reproduction (figure $6 \mathrm{c}$ et d) (JARVI et MENZIES, 1936; BACKIEL et SYCH, 1958; MAISSE et BAGLINIËRE, 1983; BAGLINIĖRE, 1985; RICHARD, 1986; MAISSE et al, 1987). L'intensité de cette marque de fraie semble dépendre du milieu, de la taille et du taux de croissance de l'individu. Ainsi, elle apparaît plus nettement chez le saumon atlantique (BAGLINIÉRE, 1985) et la truite de mer (RICHARD, 1986) que chez la truite de lac où elle peut être absente (MELHAOUI, 1985). De faux anneaux ou "checks" peuvent également être présents sur l'écaille aussi bien en phase juvénile qu'adulte (BAGLINIĖRE, 1985 ; RICHARD, 1986). le phénomène de régénération reste assez fréquent : $60 \%$ dans le cas de la truite de mer (BAGLINIĖRE et al., 1986).

La forme et la structure superficielle de l'écaille sont légèrement différentes chez les adultes de grand migrateur en raison (figure $5 \mathrm{f}$ à $\mathrm{j}$ ) :

- de la présence de marques de fraie très prononcées chez les individus de grande taille ou très âgés (deux à trois saisons de croissance entrecoupées de période de reproduction);

- de la distinction entre une phase "juvénile" (circuli plus fins et peu espacés) et une "adulte" (circuli plus épais et beaucoup plus espacés) sans qu'il y ait de convention bien établie pour séparer ces deux phases. Le phénomène est quelquefois moins net chez la truite de lac (MELHAOUI, 1985). Dans le cas d'individus à forte croissance, ces deux phases peuvent être entrecoupées d'une troisième, dite de transition (phase du juvénile migrant).

Chez ces grands migrateurs, la forme et la structure de l'écaille au stade adulte permettent de différencier les espèces du genre Salmo en considérant (figure $5 \mathrm{f}$ à j)

- la grandeur. A taille et âge égaux, l'écaille est plus petite chez la truite;

- la forme. L'écaille du saumon est plus allongée. Ce critère est mis en évidence par l’indice de forme (F) de BURDAK (1986). F- $\frac{H}{D}$ avec $H$-le plus grand diamètre transversal, et $D=$ le plus grand

diamètre longitudinal (F est d'autant plus grand que l'écaille est arrondie). La truite de mer est un poisson à fraies multiples qui entraînent notamment une érosion de la partie non recouverte de l'écaille (fig. 5 j). Ainsi ces deux caractéristiques font augmenter l'indice de forme $F$ avec l'âge chez cette espèce.

- la position du premier annulus post-migratoire. Chez la truite, il est situé beaucoup plus près du dernier arrêt de croissance observé en phase "juvénile":

- la structure des circuli en première année post-migratoire. Ceux-ci sont discontinus chez le saumon atlantique et continus chez la truite avec un espacement et une épaisseur supérieurs pour la première espèce. 
Dans cette sous-famille, la détermination de l'àge à partır des écailles reste une méthode fiable et facilement utilisable. Cependant certaines difficultés peuvent apparaître :

- chez la truite de lac (en phase adulte), en raison d'une croissance continue en liaison avec certaines conditions du milieu.

- chez le genre, Salvelinus, en raison de la petite taille des écailles et d'une croissance plus faible. Dans les régions nordiques, l'absence de premier hiver est souvent notée sur les écailles de l'omble chevalier Salvelinus alpinus Linné (GULLESTAD, 1974). De plus, pour les espèces de ce genre, en raison d'une faible croissance liée aux conditions de l'environnement. les otolithes sont souvent préférés aux écailles pour la détermination de l'âge. (JONSSON et OSTLI, 1979; FRENETTE et DODSON, 1984 ; DEMPSON et GREEN, 1985);

- chez les autres espèces, après la première reproduction mais à un degré moindre chez la truite de mer et le saumon atlantique.

\section{Sous-famille des Thymallinés, genre Thymallus et des Corégoninés. genre Coregonus} champs

L'écaille est de forme polygonale, avec la présence de radii permettant de délimiter quatre

\section{L'ombre commun Thymallus thymallus Linné.}

On distingue trois lobes de même dimension dans le champ antérieur alors que le champ postérieur est arrondi. Le réseau de circuli est déposé concentriquement autour du focus en épousant la forme de l'écaille; il est très peu marqué dans le champ postérieur (figure 7 a). Les arrêts de croissance se marquent par le resserrement de plusieurs circuli séparant deux zones de croissance différente (figure 7 b). Pour HELLAWELL (1969), l'anneau hivernal n'apparaît pas à une période correspondant à celle de la fraie et les anneaux de ponte ne seraient pas visibles. Cependant, l'observation d'écailles d'ombre du Haut-Allier et de l'Arce semble montrer que le resserrement hivernal n'est pas toujours apparu au moment de la reproduction (avril). Ainsi, un annulus peut être confondu avec un anneau de ponte. II est possible qu'un tel anneau puisse se distinguer d'un hiver sans fraie par le plus faible nombre de circuli resserrés et par leur recoupement (figure $7 \mathrm{c}$ ). Chez cette espèce, HELLAWELL (1969), MICHA (1971) et PERSAT (1977) considèrent que la détermination de l'âge par la scalimétrie reste satisfaisante.

\section{Les corégones Coregonus sp.}

En France, il y a divers hybrides plus ou moins proches de formes autochtones : $C$. lavaretus s.I. et $C$. albula introduits au lac Chauvet à partir de la pisciculture d'Huningue (KOZIKOWSKA, 1976). Chez les corégones, la classification par espèces reste difficile (MACK et BILLARD, 1984). L'espèce présentée ici est la grande fera du Léman appartenant au complexe $C$. lavaretus.

Le champ antérieur de l'écaille présente un léger dôme en son milieu avec deux radii latéraux alors qu'il existe une légère dépression dans le champ postérieur bien délimité par d'autres radii. Le focus est excentré se situant dans le tiers postérieur. Les circuli sont déposés concentriquement autour du centre de l'écaille en épousant son contour. Leur aspect permet de distinguer nettement les quatre champs. En particulier les circuli sont nettement moins serrés et quelquefois discontinus dans la partie postérieure (figure 8 a). Les liqnes d'arrêt de croissance ou annuli sont marquées par le resserrement de plusieurs circuli. Au niveau de ces structures, la discontinuité et le recoupement de circuli semblent caractéristiques de l'hiver-écaille des corégones (HOGMAN, 1968), (figure 8 b et c). Sur certains écotypes de l'espèce, des marques de fraie ont été distinguées des resserrements hivernaux habituels par suite d'un nombre plus faible de circuli (PROTOPOPOV, 1980). En période de forte croissance, on observe sur les écailles une discontinuité nette des circuli. En général chez les corégones, la détermination de l'âge à partir des écailles est tout à fait satisfaisante.

\section{FAMILLE DES ESOCIDÉS}

\section{Le brochet Esox Iucius Linné}

L'écaille de taille moyenne est de forme ovale allongée. Le focus est décentré jusqu'au tiers postérieur. Les champs ne se délimitent pas nettement mais sont malgré tout distincts (figure 9 a): - deux radii primaires rendent le champ antérieur trilobé, avec un lobe central plus développé:

- le champ postérieur est triangulaire et peu développé à cause de la proximité du focus. Les circuli y sont irréguliers.

Une striation très fine est observée, épousant le tour de l'écaille. On note une alternance annuelle de circuli espacés se traduisant par un anneau clair, puis une zone de circuli rapprochés en fin d'été donnant un anneau plus sombre. Si la croissance est médiocre ou irrégulière, l'écaille présente un aspect bigarré dû à une succession relativement rapide de circuli rapprochés et espacés. Dans les zones sombres (croissance lente), les circuli sont collés les uns aux autres et de courbure irrégulière. 
Les arrêts de croissance se marquent de différentes façons (MONGEAU, 1960)

- une ligne claire hyaline entourant toute l'écaille. C'est la marge sur laquelle ne s'est formé aucun circulus durant l'arrêt de croissance (figure 9 b):

- une ligne claire plus étroite avec une série de fragments de circuli sous forme de points ou de traits courts (figure 9 b):

- quelques circuli, rapprochés et discontinus (croissance lente), suivis de circuli complets et plus écartés (croissance rapide). Dans ce cas, l'arrêt se marque nettement mieux à la partie postérieure de l'écaille, où l'on observe une ligne claire continue (figure $9 \mathrm{c}$ ).

Notons que la distinction d'un annulus vrai se fait par la reconnaissance d'une zone de discontinuité sur l'ensemble du pourtour de l'écaille, zone qui se marque toujours mieux d'une part dans le champ antérieur à croissance générale plus rapide, d'autre part dans le champ postérieur à croissance lente : la zone claire, qui est la marque la plus nette possible d'un hiver, est due à un défaut de formation des circuli.

Les écailles régénérées sont, d'après WILLIAMS (1955), peu nombreuses par rapport à ce que I'on note chez d'autres espèces ( 1 individu sur 15 chez les jeunes poissons, 1 sur 7 chez les individus âgés).

La correspondance entre les saisons de fraie et d'arrèt de croissance ne permet pas de distinguer un hiver avec ou sans reproduction compte tenu de la structure de l'anneau hivernal.

\section{FAMILLE DES POÉCILIDES}

\section{Le gambusie Gambusia affinis Baird et Girard (figure 10).}

L'écaille de petite taille est de forme ovale étirée latéralement, plus plate à la partie orale. Le focus est déporté vers l'arrière. Le champ antérieur se marque par 10 à 12 radii primaires et secondaires, ainsi que par un plus grand nombre de circuli. Ces derniers, obliques par rapport au bord de l'écaille continuent à diminuer en nombre dans les champs latéraux, puis s'épaississent dans le champ postérieur dont les limites ne sont pas marquées. On ne peut voir d'arrêts de croissance bien que, dans le champ antérieur, des zones de circulii rapprochés indiquent une croissance ralentie. L'ensemble de deux zones claire et sombre pourrait correspondre à une année ou à un cycle reproducteur.

Le fait que cette espèce vive dans une région à climat très tempéré peut expliquer lacroissance presque continue et la difficulté de détermination de l'àge

\section{FAMILLE DES CYPRINIDÉS}

L'ablette Alburnus alburnus Linné et l'able Leucaspius delineatus Heckel. commun.

La ressemblance entre l'écaille de ces deux espèces est telle que la description peut être faite en

L'écaille est de type cycloïde simple de petite taille, de forme ovalaire étirée dans le sens latéral. Aucun lobe ni ornementation ne s'y remarque. Le focus est déporté vers l'avant. Les champs ne se distinguent que par la distribution de radii peu marqués. Chez l'ablette (figure 11 a), on note une dizaine de ces radii (jusqu'à 12) dans le champ postérieur, un peu moins dans le champ antérieur. Certains sont de type secondaire, n'allant pas jusqu'au centre et semblent se développer à partir d'une reprise de croissance. Par contre, chez l'able (figure 12). on n'observe que deux radii dans le champ antérieur.

Chez les deux espèces, l'arrèt de croissance se marque par une bande claire et un recoupement des circuli dans les champs latéraux et antérieur (figure 11 b). Cette bande claire s'élargit dans le champ postériéur. Au cours de l'année, aucune différence entre l'espacement des circuli n'est apparente.

\section{Le goujon Gobio gobio Linné}

La forme générale de l'écaille est en fer à cheval, avec un champ antérieur à trois lobes peu marqués et à bordure rectiligne. Le focus est déplacé vers l'avant. Les champs sont très bien délimités (figure 13 a):

- I'antérieur très peu développé avec des circuli parallèles au bord de l'écaille:

- le champ postérieur de teinte très claire montrant des circuli nombreux mais peu proéminents et une vingtaine de stries radiaires. La plupart sont de type primaire plus ou moins en continuité avec les circuli des champs latéraux. Très développé, ce champ occupe la moitié de la surface totale :

- Les champs latéraux, bien délimités, montrent des stries obliques par rapport au bord de l'écaille.

Les arrêts de croissance se marquent sur les champs latéraux par une chaine ondulée due aux discontinuités de formation de circuli (figure 13 b). La reprise entraine quelques recoupements (BERNET, 1960). A l'arrière, on observe une ligne fine plus sombre recoupant les stries radiaires. Le 
champ antérieur marqué par une zone plus sombre ne permet d'observer que rarement une diminution de croissance en fin de saison. Le premier annulus est parfois placé très près du centre de l'écaille (CERVIA et TORRES, 1983).

La détermination de l'âge par scalimétrie chez cette espèce n'est pas toujours évidente (OMBREDANE, données non publiées).

\section{L'ablette spirlin Alburnoides bipunctatus Bloch.}

La forme générale de l'écaille est proche de celle du goujon quant à l’importance respective des champs. Les lobes sont moins marqués à l'avant, et le champ postérieur plus étroit ne montre que 6 à 8 stries radiaires. La taille est petite (figure 14 a).

Les arrêts de croissance se marquent sur les champs latéraux par le recoupement de quelques circuli seulement (figure 14 b). Un de ceux-ci souligne chaque hiver dans le champ postérieur.

La carpe herbivore Ctenopharyngodon idella Valenciennes.

L'amour blanc ou carpe herbivore est une espèce introduite en pisciculture depuis 1964 (ALLARDI, 1982).

L'écaille rappelle en plus grand celle du goujon (figure 15 a). La partie postérieure est plus développée et les champs sont bien délimités. Le champ antérieur à bords ondulés est parcouru par une douzaine de radii et limité par deux excroissances latérales. Le champ postérieur possède 10 à 12 radii primaires.

Dans les champs latéraux, les circuli parallèles au bord deviennent obliques vers l'arrière de la zone alors que le phénomène s'inverse dans le champ postérieur au cours de la croissance.

Les arrêts de croissance se marquent dans les champs antérieur et latéraux par un resserrement assez net des circuli (figure 15 b)

\section{La carpe argentée Hypophthalmichthys molitrix Valenciennes.}

Cette espèce a été introduite récemment en pisciculture. La forme et la structure de l'écaille est très semblable à celles des Salmonidés du genre Salmo (figure 16 a et voir figures 5 a à J). L'écaille est de petite taille et de forme ronde régulière. Seul le champ postérieur se distingue par un changement d'orientation des circuli qui deviennent obliques par rapport au bord de l'écaille et moins nombreux.

Les arrêts de croissance se marquent dans le champ antérieur de l'écaille par un resserrement des circuli. Cependant, comme dans la famille des Salmonidés, le premier anneau hivernal se repère par un changement net de l'espacement des circuli dans le champ antérieur (figure 16 b). De plus, on observe un recoupement bien net des circuli dans le champ postérieur, les premiers formés la seconde année épousant le pourtour de l'écaille, en discordance avec la direction oblique des précédents.

Pour les trois espèces qui vont suivre, rattachées aux genres Tinca et Barbus, la forme et la structure superficielle des écailles ont des caractères communs : allongement dans le sens antéropostérieur et présence de nombreux radii. Par ailleurs la détermination de l'âge par scalimétrie apparaît plus difficile (MICHA, 1971 ; KRAIEM, 1979).

\section{La tanche Tinca tinca Linné.}

Les écailles de taille moyenne sont deux fois plus longues que larges, avec un focus très déporté vers l'arrière. Les champs ne se distinguent pas les uns des autres, l'écaille est plus large à l'avant. Les stries radiaires, primaires et secondaires, sont très nombreuses (figure 17 a).

Des discontinuités de croissance, pouvant marquer les années, sont représentées par des zones plus claires formant des anneaux complets (figure $17 \mathrm{~b}$ ).

\section{Le barbeau commun Barbus barbus Linné}

L'écaille est de forme allongée, pointue à l'arrière, de taille moyenne. Le focus est central. Des stries radiaires s'observent sur toute la surface (figure 18 a).

Le champ antérieur est unilobé. Les champs latéraux se marquent par des radii obliques. Outre sa forme en pointe, le champ postérieur se distingue par le faible nombre de circuli, deux à trois fois moins nombreux que sur le reste de l'écaille.

Les arrêts de croissance se marquent par une alternance de zones sombre et claire, et par un recoupement des circuli dans les champs latéraux (figure 18 b).

\section{Le barbeau méridional Barbus meridionalis Risso.}

L'écaille est de même forme et de mème taille que celle de l'espèce précédente. On remarque simplement que le champ antérieur n'est presque pas lobé et que la partie orale paraît ainsi plate. Les stries radiaires sont plus nombreuses (une soixantaine, surtout primaires) et se distribuent uniformément sur toute la surface (figure 19). 
Pour les dernières espèces de Cyprinidés étudiées : gardon-rotengle, hybride gardon-rotengle. hotu, soffie, vandoıse, chevaine, brème bordelière, poisson rouge, carpe, brème commune et carassin, à l'exception de la carpe miroir et du vairon, les écailles ont des caractéristiques communes

L'écaille est grande, de forme arrondie avec des champs bien marqués. La partie antérieure est multilobée ou ondulée et souvent dissymétrique. Dans ce cas c'est le flanc dorsal qui est le plus développé. Des radii soulignent le champ postérieur. Les distances entre circuli sont très variables suivant le rythme de croissance, et les années se marquent par une alternance de zones claires et sombres

On note parfois des érosions de l'écaille correspondant pour certains auteurs à une résorption au moment de la reproduction, donc à une marque de fraie (HARTLEY, 1947: WILLIAMS, 1967; JONES, 1953 in PHILIPPART, 1971). Pour LIEDER (1969), cela est lié à de mauvaises conditions hivernales. WALLIN (1958) reproduit cette structure chez le gardon en provoquant des avitaminoses. On peut cependant distinguer chacune des espèces par des caractères précis.

\section{Le gardon Rutilus rutilus Linné.}

Les écailles sont en forme de fer à cheval, les bords parallèles. Le focus est central. Les champs ne sont pas très marqués, bien que distincts (figure 20 a).

Le champ antérieur trilobé est limité par deux excroissances dissymétriques. Les stries radiaires y sont particulièrement marquées.

Le champ postérieur à borcis ondulés montre des circuli moins nets mais aussi nombreux.

Ceux-ci sont parallèles au bord de l'écaille. Les alternances "zone claire" (croissance rapide) "zone sombre" (croissance ralentie) sont moins marquées que ce qu'indiquent SAEED et LIBOSVARSKY (1983). Dans les champs latéraux et antérieur, les annuli forment des chaînes ondulées (figure 20 b). Dans le champ postérieur où les circuli sont moins nets, on observe un anneau plus sombre marquant probablement la reprise de croissance. II n'y a pas de recoupement des circuli. Contrairement à ce que note LINFIELD (1974), l'érosion de la partie latéro-antérieure de l'écaille en hiver n'est pas la règle générale.

Le rotengle Scardinius erythrophthalmus Linné (figure 21).

Les écailles se distinguent de celles du gardon par une forme plus arrondie: les bords des champs latéraux ne sont pas vraiment parallèles. On y observe un recoupement des circuli. Les lobes du champ antérieur sont moins marqués. Les circuli sont plus espacés dans le champ postérieur que dans le reste de l'écaille.

Les arrêts de croissance se marquent de la même façon que chez le gardon. Cette espèce a servi pour des tests de lecture de l'âge et de retro-mesure (MANN et STEINMETZ, 1985).

\section{L'hybride gardon-rotengle (figure 22).}

Aucune différence ne s'observe comparativement au gardon.

Le hotu Chondrostoma nasus Linné (figure 23).

L'écaille est de forme carrée avec le focus déporté vers l'avant, presque au premier tiers. marquées.

Le champ antérieur est bilobé et les deux excroissances latérales, dissymétriques, sont peu

Le champ postérieur paraît de teinte plus claire.

On ne note pas deux types de croissance au cours de l'année. Les marques hivernales sont formées par une ligne concentrique due à un rapprochement de deux à trois circuli et à un recoupement lisible sur une grande partie des champs latéraux. Dans le champ postérieur, cet annulus apparaît sous la forme d'une ligne plus épaisse.

Si en général les écailles restent bien lisibles chez cette espèce, il existe quelquefois des difficultés liées à l'âge, au sexe (plus difficile chez les mâles) ou au taux de croissance de la population (NELVA-PASQUAL, 1985). De plus, d'après cet auteur, le premier annulus ne serait pas marqué.

\section{La soffie Chondrostoma toxostoma Vallot (figure 24).}

L'écaille est très comparable à celle du hotu quant à sa forme et à sa structure (focus vers l'avant, radii, excroissances latérales). La partie antérieure est pourtant un peu plus bombée.

Les marques d'arrêts sont de même type, mais on note en plus deux types de croissance au cours de l'année, particulièrement lisibles dans les champs latéraux.

La vandoise Leuciscus leuciscus Linné.

L'écaille est ovale, étirée dans le sens latéral (figure 25 a). 1971).

Le focus est au centre de l'écaille, déporté vers l'avant chez les poissons âgés (PHILIPPART,

Le champ antérieur possède des lobes très peu marqués, la partie orale paraît presque plate, forme soulignée par les deux excroissances latérales étirées sur les côtés. Les radii y sont très nombreux (une vingtaine, primaires et secondaires) surtout de part et d'autre des lobes. 
Le champ postérieur est multilobé comme chez les autres espèces, avec de 6 à 10 stries radiaires. Les circuli y sont deux à trois fois moins nombreux que sur les champs latéraux.

Les arrêts de croissanç se marquent par un recoupement des circuli à la partie latéropostérieure et par une zone hyalıne soulignée par des circuli irréguliers (figure 25 b).

Une étude bibliographique sur la scalimétrie de cette espèce est faite par PHILIPPART (Joc. cit.).

\section{Le chevaine Leuciscus cephalus Linné} 26 a)

L'écaille est ovale. légèrement étirée dans le sens antéro-postérieur. Le focus est central/figure

Le champ antérieur à bordure plus ou moins rectiligne présente quatre lobes peu marqués et deux excroissances latérales bien distinctes. On $y$ voit une vingtaine de radii.

Le champ postérieur multilobé montre des radii primaires et secondaires (sept ou huit de chaque sorte). Les circuli y sont deux fois moins nombreux que dans les champs latéraux.

Les années se marquent par une alternance de zones claires et sombres, particulièrement dans le champ antérieur (PHILIPPART, 1972), et dans tout le champ latéral par un recoupement très prononcé des circuli (figure 26 b). Ceux-ci sont parfois irréguliers en début de saison (PHILIPPART, Ioc. cit. ; PROKES et al. 1977). Des érosions peuvent se rencontrer sur les écailles de poissons àgés et des dépôts osseux peuvent masquer le premier annulus (LIBOSVARSKY, 1956). Dans ce cas, on peut le situer par un certain recoupement (RICHARD, 1973). En outre, le premier circulus formé au printemps lors de la reprise de croissance est parallèle au bord postérieur de l'écaille (figure 26 c).

\section{La brème bordelière Blicca bjoerkna Linné.}

L'écaille est aussi large que longue et de forme générale irrégulière (excroissances latérales développées et dissymétriques, champs latéraux à bords convexes). Le focus est au centre de l'écaille (figure 27 a).

Le champ antérieur esı bien marqué par les deux excroissances latérales et par deux lobes centraux dont les sillons sont soulignés par de nombreux radii secondaires.

Un champ postérieur, à sept-huit lobes séparés par des radii primaires montre en plus de nombreux radii secondaires. On y voit autant de circuli que dans les champs latéraux.

Les années se marquent par une alternance de zones claires et sombres. On n'observe pas de recoupement des circuli, mais une ligne claire faisant le tour de l'écaille est l'indication la plus nette d'un arrèt hivernal (figure 27 b).

\section{Le poisson rouge Carassius auratus Linné}

La forme générale s'apparente à un carré, le focus est placé légèrement vers l'arrière. Les champs sont bien marqués (figure 28 a).

Le champ antérieur est bordé par deux excroissances dissymétriques aigües et cinq lobes en dents de scie marquant la partie orale. Les six radii primaires soulignent les échancrures.

Quelques radii primaires existent également dans le champ postérieur. Ce dernier est multilobé et les circuli y sont deux fois moins nombreux que dans les champs latéraux.

Ceux-ci sont bien délimités. Les circuli sont obliques par rapport au bord de l'écaille dans la partie postérieure.

Les arrèts de croissance se marquent de différentes manières : à la partie orale par un resserrement des stries (écrasement) et une discontinuité. dans la partie latérale par un recoupement, dans le champ postérieur par un changement d'orientation très marqué des circuli (figure 28 b).

\section{La carpe Cyprinus carpio Linné (figure 29)}

Le champ antérieur est limité par deux lobes latéraux peu marqués el quelques ondulations de la partie orale. On y voit de 25 à 30 radii (primaires et secondaires).

Le champ postérieur esı en forme de triangle large marqué également par des stries radiaires nombreuses. Les circuli sont deux ou trois fois moins nombreux que dans les champs latéraux.

Les arrêts de croissance se marquent en général par une zone relativement large de circuli fragmentés et rapprochés et par un recoupement à la partie latérale postérieure.

Les écailles de carpe miroir sont de forme très irrégulière, souvent en éventail (figure 30).

La brème commune Abramis brama Linné.

L'écaille est ovale, étirée dans le sens latéral, le focus est au centre (figure 31 a).

Le champ antérieur est trilobé à sillons très peu marqués comme les excroissances latérales. La forme arrondie en est soulignée.

Le champ postérieur possède 6 à 10 radii. Les circuli y sont aussi nombreux que dans les champs latéraux (GAJDUSEK, 1981). 
Les annuli ne se marquent pas par des recoupements, mass par un changement dans l'espacement intercirculi entraînant une alternance très nette de croissance rapide et lente. Une ligne hyaline souligne les arrêts dans le champ postérieur (figure 31 b).

Le carassin Carassius carassius Linné (figure 32).

L'écaille est légèrement étirée dans le sens latéral. Le focus est un peu déporté vers l'arrière.

Le champ antérieur est très peu lobé et limité par deux excroissances latérales dissymétriques. On y observe quelques radii.

Le champ postérieur est marqué par 8 à 12 radii. Les circuli y sont moins nombreux.

Les arrêts de croissance sont soulignés par des zones de circuli irréguliers. II n'y a pas de réels recoupements dans les champs latéraux. L'écaille est en bien des points différente de celle de l'autre espèce de Carassius (figure 25).

\section{Le vairon Phoxinus phoxinus Linné (figure 33).}

L'écaille est très différente de celle des autres espèces précédemment décrites. Elle est très petite et presque circulaire, le focus est placé au tiers antérieur.

Les champs sont peu distincts. Une dizaine de radii primaires occupent le champ antérieur. Dans les zones latérales, ces radii sont obliques. Ils redeviennent rectilignes dans le champ postérieur marqué également par la raréfaction des circuli.

On ne peut distinguer de discontinuités de croissance.

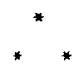

Les écailles des espèces des trois dernières familles étudiées (Percidés, Mugilidés et Centrarchidés) sont de type cténoïde et présentent des traits communs: taille moyenne, forme générale rectangulaire, champs bien délimités. Par contre, l'ornementation du champ postérieur permet de distinguer les deux premières familles de la dernière. Ainsi chez les Percidés et les Mugilidés le champ postérieur ne présente pas de circuli et est recouvert de ctenii ; chez les Centrarchidés, quelques circuli y sont présents et les spinules sont situées sur le bord libre de l'écaille.

\section{FAMILLE DES PERCIDÉS}

Chez les représentants de cette famille, l'épaisseur relativement importante de l'écaille a entraîné l'utilisation d'autres structures osseuses pour la détermination de l'âge : os operculaire (LECREN, 1947), rayons de nageoires (GERDEAUX, 1986).

\section{Le sandre Stizostedion lucioperca Linné.}

L'écaille est presque carrée. Le champ antérieur est formé de six à huit lobes y compris les excroissances latérales peu marquées. Des radii profonds les séparent. Les circuli y sont deux fois plus nombreux que dans les champs latéraux (figure 34 a).

Le champ postérieur est séparé des champs latéraux par une limite rectiligne. Les arrêts de croissance se marquent différemment suivant les parties de l'écaille: dans le champ antérieur, on remarque un resserrement de quelques circuli; dans les champs latéraux, dès le deuxième hiver, ils apparaissent soit sous forme de bandes claires, soit précédés par un recoupement de circuli (figure $34 \mathrm{~b}$ et $34 \mathrm{c}$ ).

\section{La perche Perca fluviatilis Linné.}

L'écaille est étirée dans le sens antéro-postérieur. Cinq sillons (parfois six) séparent les lobes profondément découpés du champ antérieur. Les champs latéraux sont arrondis (convexes), rétrécis à la partie postérieure. Le nombre de circuli est le même dans les champs antérieur et latéraux, au contraire du sandre (figure 35 a).

Les arrêts hivernaux se marquent par un resserrement des stries et par un certain décollement du premier circulus lors de la reprise de croissance (figure 35 b).

\section{La grémille Gymnocephalus cernua Linné}

La forme générale de l'écaille est trapézoidale, la plus grande dimension est offerte par la partie orale. Les champs sont très bien marqués (figure 36 a).

Le champ antérieur montre de six à neuf lobes à circuli très fins. Ces derniers y sont deux fois plus nombreux que dans les champs latéraux, se rejoignant vers le focus. Le champ postérieur n'est pas totalement ornementé. Les spinules occupent toute la bordure. Le caractère cténoïde n'apparaît donc pas dès le début de la croissance de l'écaille.

Les marques d'arrêts hivernaux ne sont pas facilement distinguables à l'exception de possibles resserrements de circuli visibles seulement dans le champ antérieur (figure 36 b). 


\section{FAMILLE DES MUGILIDÉS}

Cette famille comprend des poissons amphibiotiques se reproduisant en mer et migrant en eau douce ou en eau saumâtre plus ou moins périodiquement au cours d'une année. Sa classification par espèces au niveau européen reste difficile et a pu ètre établie en considérant soit la forme des otolit hes (LAM HOAI THONG, 1969), soit le nombre de caeca pyloriques (LAM HOAI THONG, 1969 ; ALBERTINIBERHAUT, 1978). Actuellement, il peut être considéré qu'il existe deux genres, Liza et Chelon avec trois espèces communes en France: $L$. ramada Risso, $L$. aurata Risso et $C$. labrosus Risso (DUBUIT et BEAUPOIL, 1985). Ces deux dernières espèces sont présentées ici (figure 37 a et b).

Une description des écailles des muges armoricains a déjà été réalisée par LAM HOAI THONG (1969). Elles ont un aspect d'écusson (polygonal). L'ornementation principale consiste en une striation sensiblement parallèle à leur bord. Les quatre directions de stries déterminent quatre champs. Les circuli sont présents uniquement dans les champs latéraux et antérieur. Au fur et à mesure que le poisson grandit, le nombre de circuli devient plus important dans le champ antérieur, et le focus accompagné de la dépression muqueuse présente dans la partie médiane de l'écaille, migre dans le champ postérieur. Chez les muges âgés, le focus et les circuli de la zone centrale disparaissent, laissant place à un espace scaliforme parsemé de granulations irrégulières. Des radii primaires (maximum 10) sont présents dans le champ antérieur. Dans la partie postérieure, on peut distinguer une bande externe à ctenii (rangée de spinules pointues) et une zone interne à spinules émoussées.

Le ralentissement hivernal peut être défini par une bande étroite à circuli resserrés (LAM HOAI THONG, 1969) avec un recoupement plus ou moins prononcé (LIBOSVARSKY, 1976). Le nombre de ces anneaux permet d'estimer l'àge qui ne pourra être connu avec précision qu'en tenant compte des dates de ponte, d'éclosion et de capture (LAM HOAl THONG, 1969) (figure $37 \mathrm{c}$ ). L'espèce $L$. aurata pondrait en automne (ALBERTINI-BERHAUT, 1981) et $C$. lebrosus au début du printemps (LAM HOAI THONG, 1969) ce qui fait que sur l'écaille, périodes de ponte et de ralentissement de croissance peuvent être confondues. Par ailleurs, il semble exister un phénomène d'occultation du premier annulus mis en évidence chez $L$. ramada (QUIGNARD et AUTHEM, 1981). Cependant, la scalimétrie reste pour cette famille la méthode la plus utilisée pour la détermination de l'àge en raison de la difficulté d'utilisation et d'interprétation de leurs otolithes (LAM HOAI THONG, 1969).

\section{FAMILLE DES CENTRARCHIDÉS}

\section{La perche-soleil Lepomis gibbosus Linné (figure 38).}

Ressemblant à celle des Percidés avec un focus déporté vers l'arrière, l'écaille se distingue par la morphologie du champ postérieur. Elle est de forme rectangulaire étirée dans le sens latéral. Les champs sont bien marqués. La taille est moyenne. découpés.

Dans le champ antérieur. 12 à 15 sillons délimitent des lobes étroits et peu profondément

Le champ postérieur montre une zone centrale épineuse de forme triangulaire dont la pointe arrive au focus. De part et d'autre de cette zone, des circuli largement espacés non parallèles au bord de l'écaille. deux ou trois fois moins nombreux que dans les zones latérales, font la liaison avec les champs latéraux relativement étroits

Les annuli se marquent de deux façons:

- le premier par un décollement net du premier circulus lors de la reprise de croissance. En outre, durant la première année, les circuli sont beaucoup plus nombreux dans le champ antérieur que dans les champs latéraux:

- les suivants par un décollement à la partie latéro-antérieure, deux ou trois circuli très rapprochés dans les champs latéraux et un faible recoupement à la partie latéro-postérieure.

\section{Le black-bass Micropterus salmoides Lacépède.}

La forme générale de l'écaille est ronde, même la partie orale est convexe. Comme chez la perche-soleil, le focus est déporté vers l'arrière. La taille est assez grande (figure 39 a).

Le champ antérieur est marqué par 6 à 10 sillons. Le champ postérieur comprend les deux parties décrites pour l'espèce précédente.

Les arrêts de croissance se marquent dans le champ antérieur par une ligne claire, dans les parties latéro-postérieures par des recoupements de circuli (figure 39 b). D'après WURTZ-ARLET (1952) et ALLARDI (1973), ce dernier critère est le plus typique pour la détermination de l’âge. 


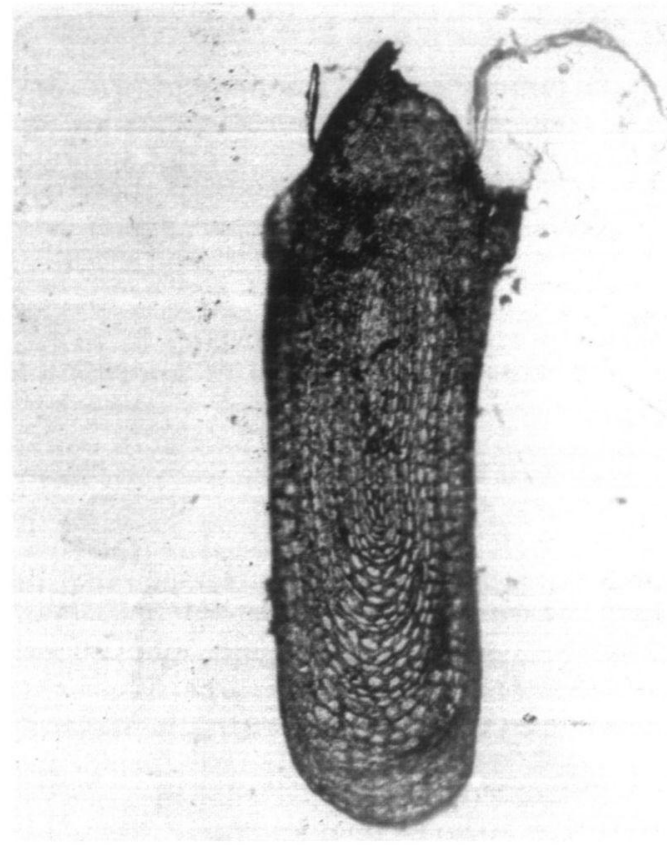

Figure 1a : Écaille d'anguille (x 50).

Estuaire du Blavet (Bretagne). LT : $450 \mathrm{~mm}$.

Figure 1a : Eel scale (x 50).

Estuary of Blavet (Brittany). TL : $450 \mathrm{~mm}$.

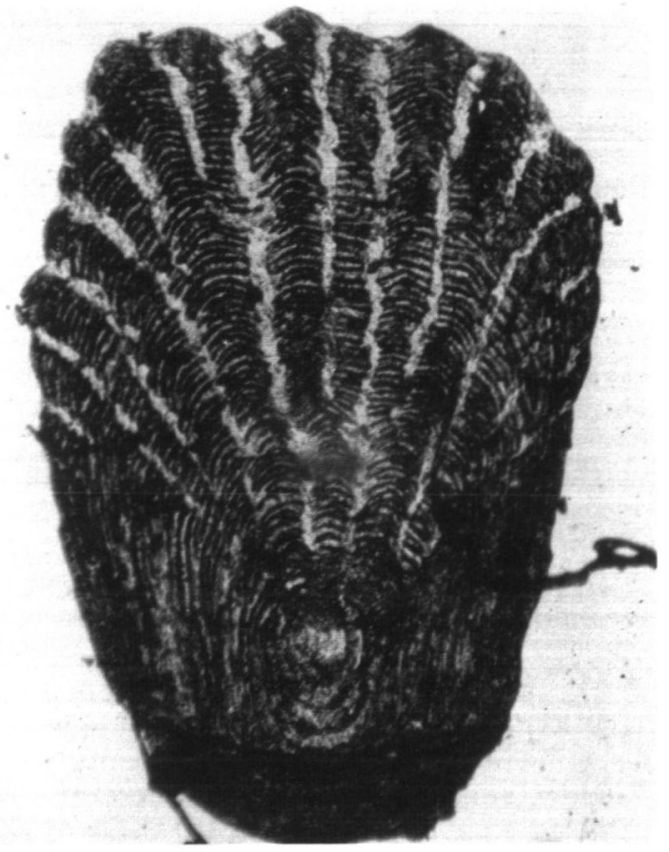

Figure 1b: Écaille de flet (x 100).

Estuaire du Scorff (Bretagne).

Capture : 17.01.84.

LF : $200 \mathrm{~mm}$.

Figure 1b: Flounder scale (x 100).

Estuary of Scorff (Brittany).

Catch : 17.01.84.

FL : $200 \mathrm{~mm}$.

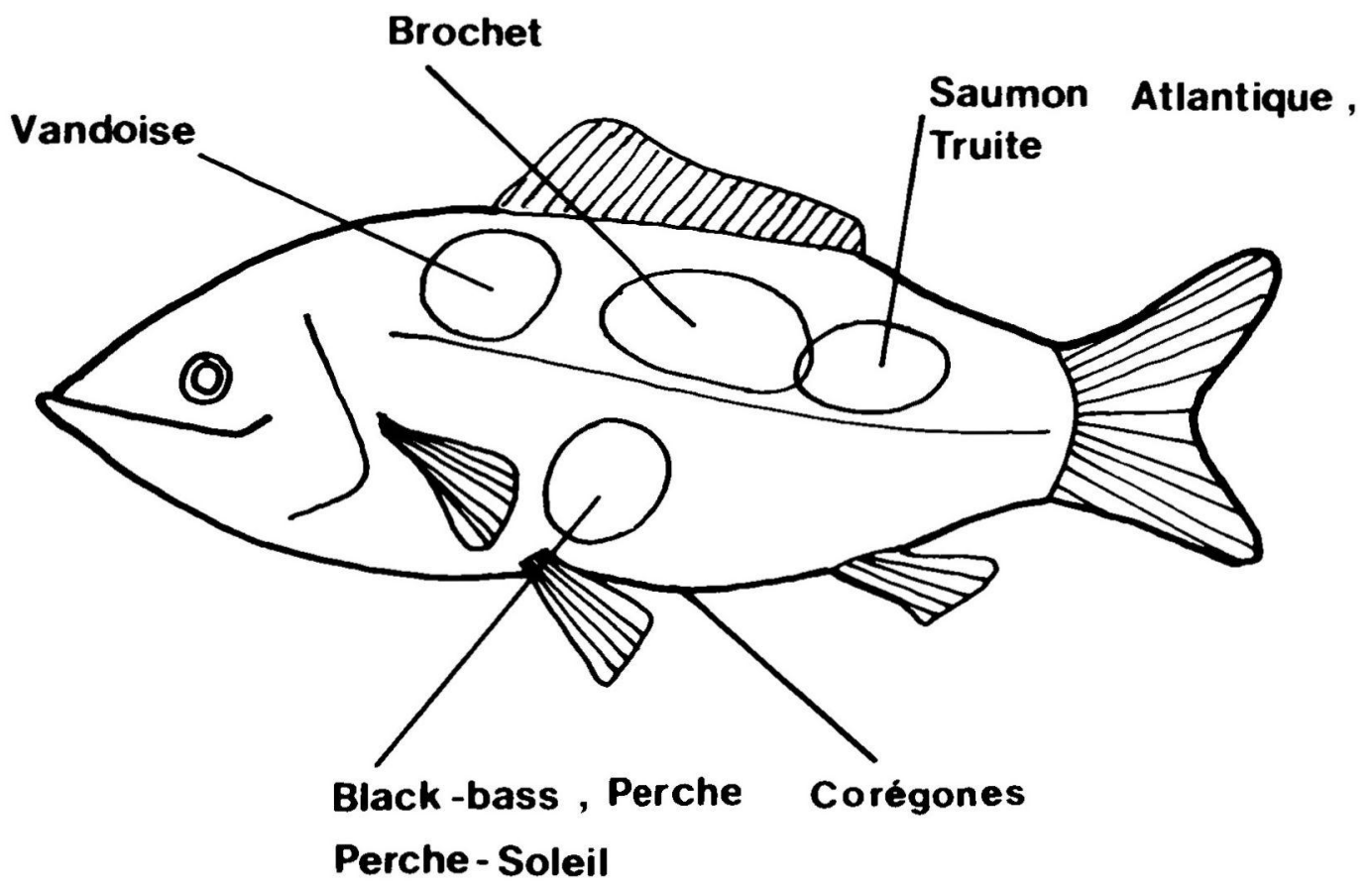

Figure 2 : Localisation des prises d'écailles chez les différentes espèces. (D'après CASSELMANN, 1967 ; JEARLD, 1983 ; BAGLINIÉRE, 1985 ; RICHARD, 1986 : CHAMPIGNEULLE, com. pers.).

Figure 2 : Standard zone of scales removing in some species (CASSELMANN, 1967 ; JEARLD. 1983 ; BAGLINIERE. 1985 ; RICHARD. 1986 ; CHAMPIGNEULLE, com. pers.). 


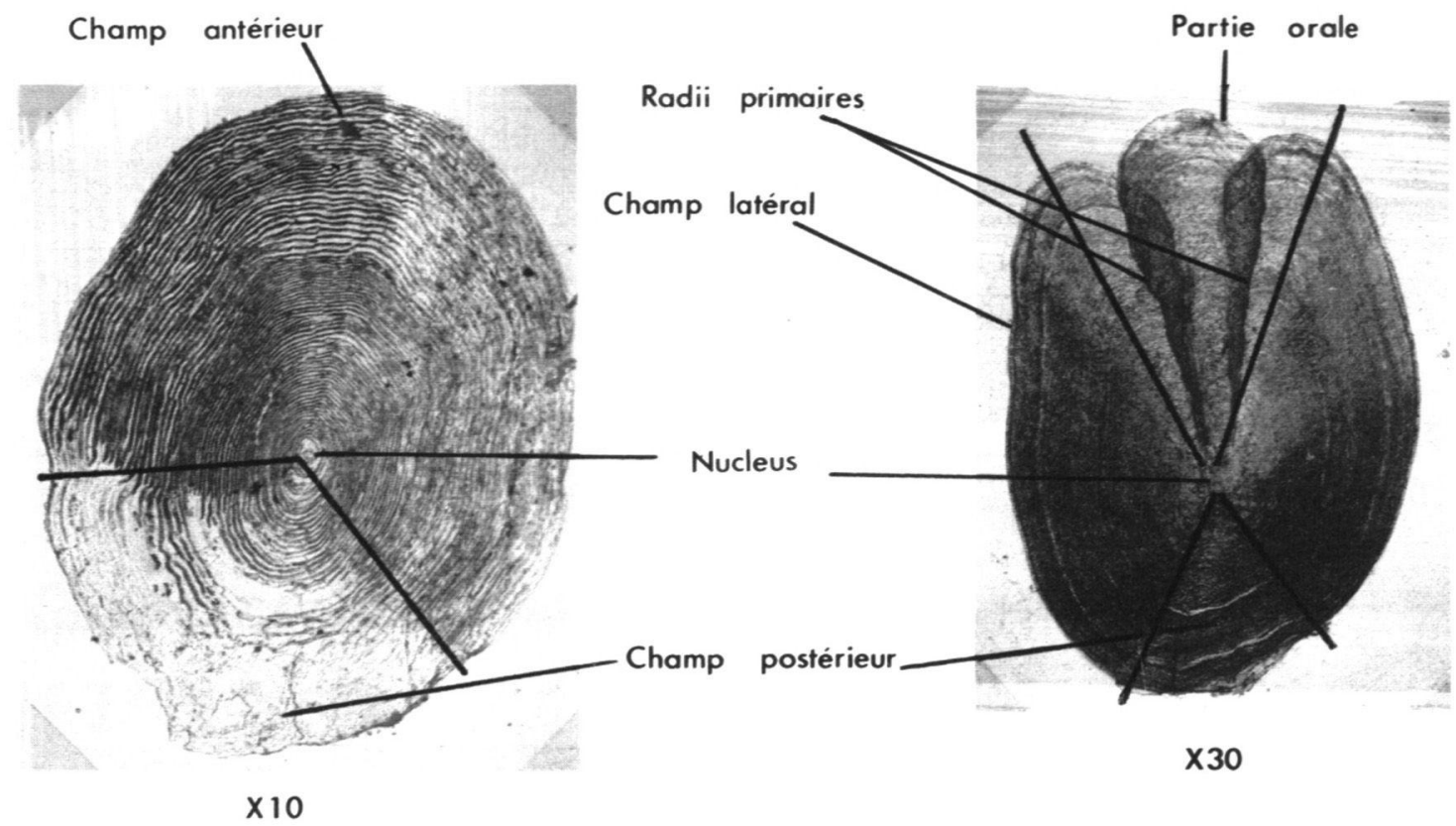

A

B

Figure 3 : Écaille de truite (A) et de brochet (B) montrant l'existence de 4 champs distincts chez la première espèces. et l'individualisation du champ postérieur chez la seconde.

Figure 3 : Trout (A) and pike (B) scale, showing 4 distinctiv parts on the first and 2 on the second.

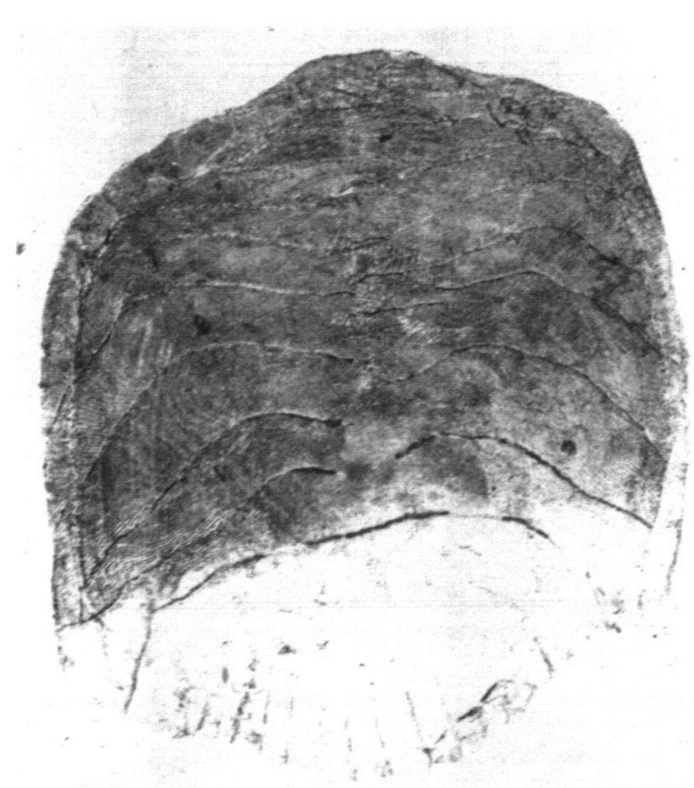

Figure 4a: Écaille de grande alose (x 10).

Côte atlantique.

Capture : 6.07.80 - Age : 6 .

Figure 4a: Shad scale (x 10).

Atlantic coast.

Catch : 6.07.80 - Age : 6 .

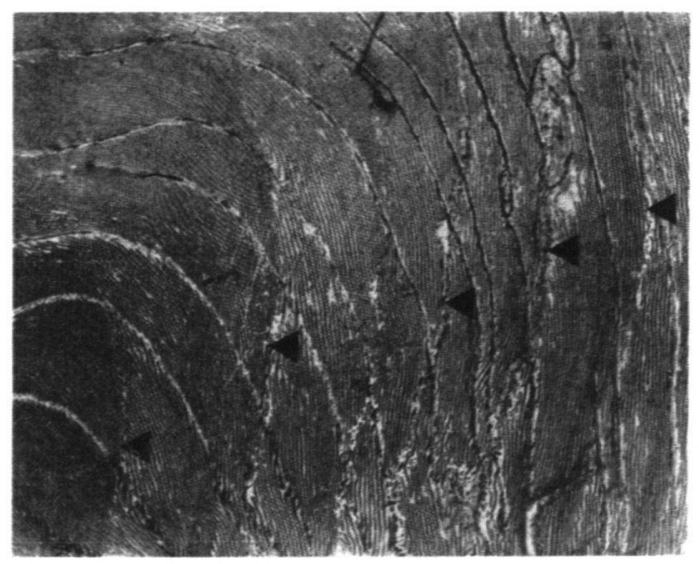

Figure 4b: Anneaux hivernaux sur une écaille de grande alose (x 30) (4).

Figure 4b: Winter bands on shad scale (x 30) (4). 


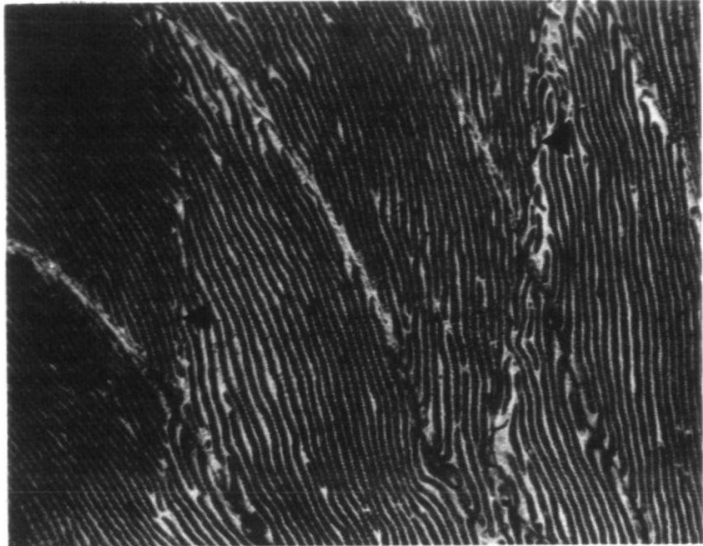

Figure 4c: Détail d'un anneau hivernal sur une écaille de grande alose $(\downarrow)$ : changement d'épaisseur et/ou discontinuité des circuli (x 50).

Figure 4c: Winter band on shad scale $(\downarrow)$ : thickness change and/or cutting-over of circuli (x 50).

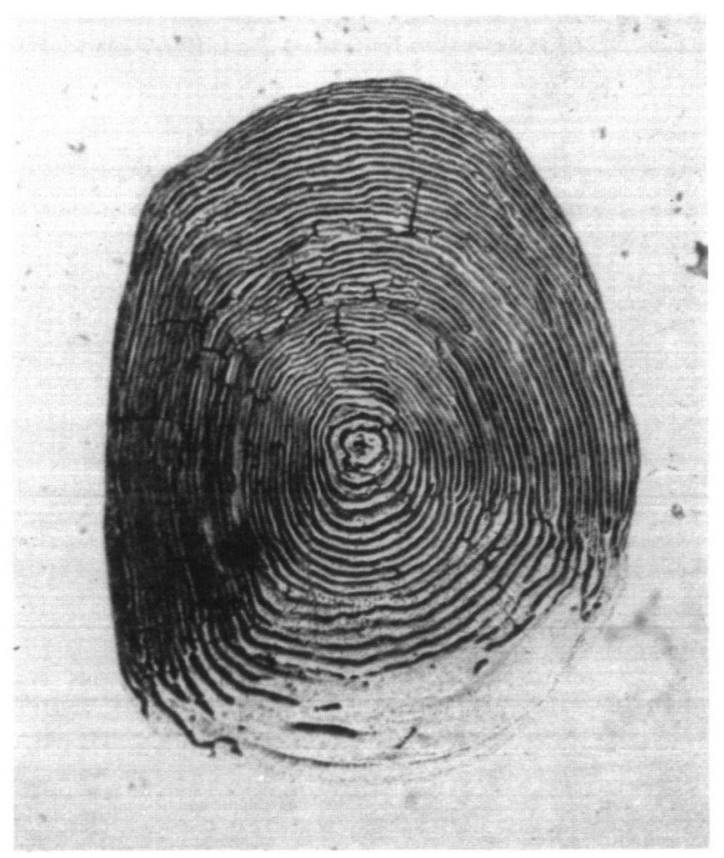

Figure 5b : Écaille de truite arc-en-ciel ( $x 30$ ) Pisciculture de Rennes (Bretagne).

Capture : 14.03.86.

Poids : $200 \mathrm{~g}-\delta$ - Age 1.

Figure 5b : Rainbow trout scale (x 30). Hatchery of Rennes (Brittany). Catch : 14.03 .86 Weight : $200 \mathrm{~g}-\delta$ - Age 1 .

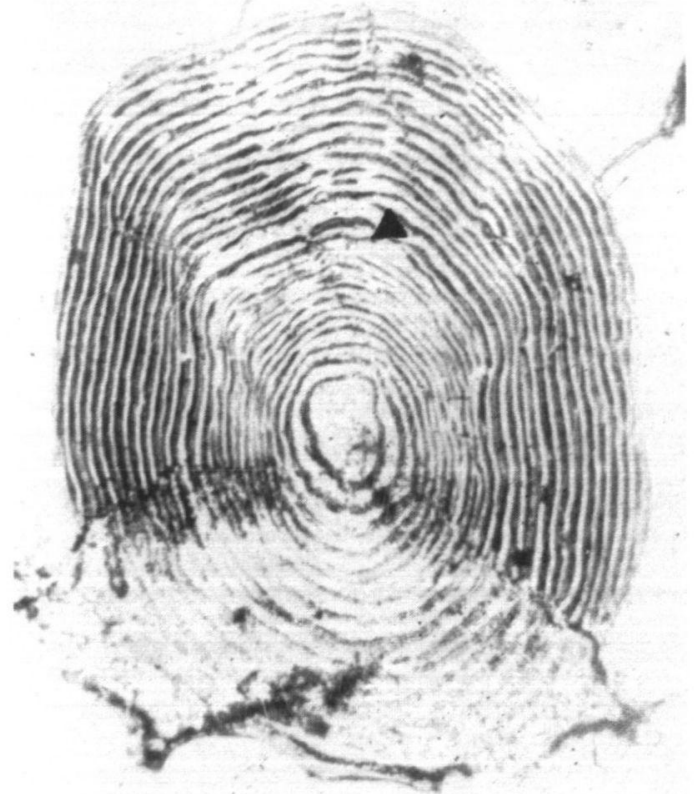

Figure 5a: Écaille de truite commune (x 50) Colmont (Mayenne).

Capture : 4.10.78.

LF : $160 \mathrm{~mm}$ - Age : $1+$.

Anneau hivernal $\bullet$.

Figure 5a : Brown trout scale (x 50). Colmont (Mayenne).

Catch : 4.10.78.

$\mathrm{FL}: 160 \mathrm{~mm}$ - Age : $1+$. Winter band $\bullet$.

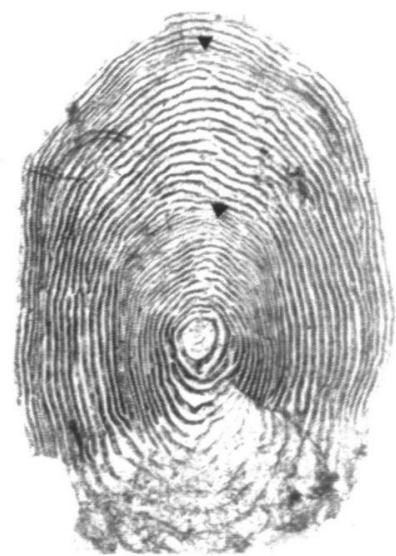

Figure 5c: Écaille de smolt de saumon (x 50).

Ruisseau de Kernec (Bretagne). Capture : 6.04.85.

LF. : $140 \mathrm{~mm}$ - ? Age : $2+$.

Anneau hivernal $\bullet$.

Figure 5c: Atlantic salmon smolt scale (x 50).

Kernec Brook (Brittany).

Catch : 6.04.85.

FL : $140 \mathrm{~mm}$ - \& Age : $2+$.

Winter band $\bullet$. 


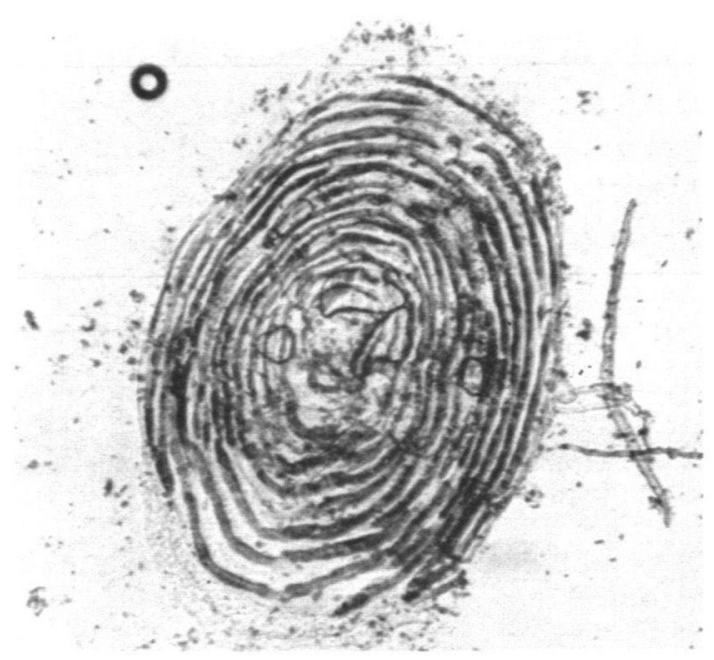

Figure 5d : Écaille d'omble de fontaine (x 100).

Pisciculture de Gournay (lle de France).

Capture : 04.86.

LF : $182 \mathrm{~mm}$ - Age : $1+$.

Figure 5d: Brook trout scale (x 100). Hatchery of Gournay (Ile de France).

Catch : 04.86 .

FL : $182 \mathrm{~mm}$ - Age : $1+$.

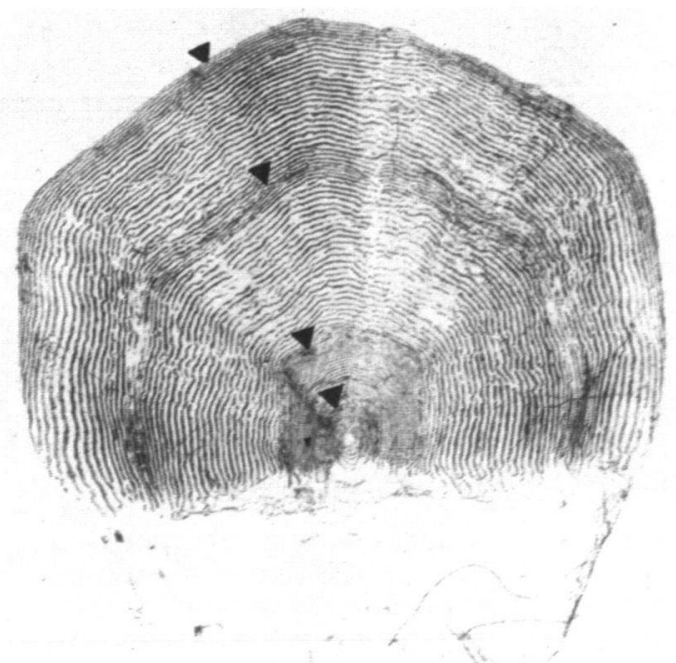

Figure 5f: Écaille de saumon atlantique (x 10).

Scorff (Bretagne).

Capture : 7.03.81.

LT : $920 \mathrm{~mm}$ - Age : 2.2 .

Anneau hivernal $\bullet$.

Figure 5f : Atlantic salmon scale (x 10).

Scorff (Brittany).

Catch : 7.03.81.

TL : $920 \mathrm{~mm}$ - Age : 2.2 .

Winter band $\bullet$.

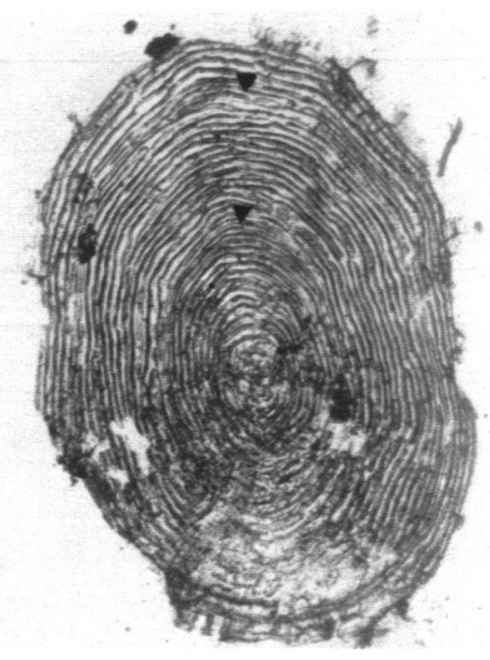

Figure 5e: Omble chevalier (x 50).

Pisciculture de Thonon (Lac Léman).

Capture : 10.09.85.

LF : $277 \mathrm{~mm}$ - Age : 2 i.

Anneau hivernal $\bullet$.

Figure 5e : Arctic char scale (x 50).

Hatchery of Thonon (Lake Leman).

Catch : 10.09.85.

FL : $277 \mathrm{~mm}$ - Age : 2 t.

Winter band $\bullet$.

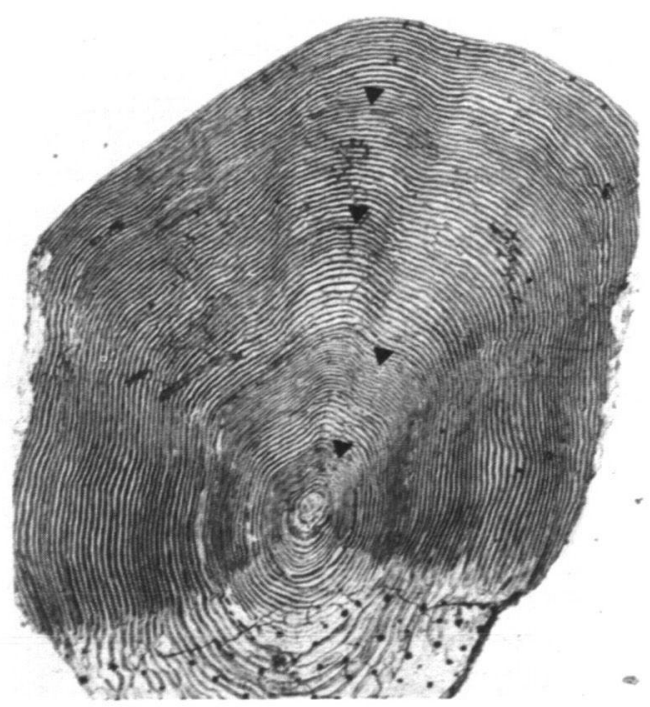

Figure 5g: Écaille de truite de lac (x 10).

Lac Léman.

Capture : 19.12.83.

LT : $780 \mathrm{~mm}$ - Age : $2+$ en ruisseau. 2 + en lac.

Anneau hivernal $\bullet$.

Figure 5g : Lake trout scale (x 10)

(Lake Leman).

Catch : 19.12.83.

TL : $780 \mathrm{~mm}$ - Age : $2+$ years in tributary. $2+$ in lake.

Winter band $\bullet$. 


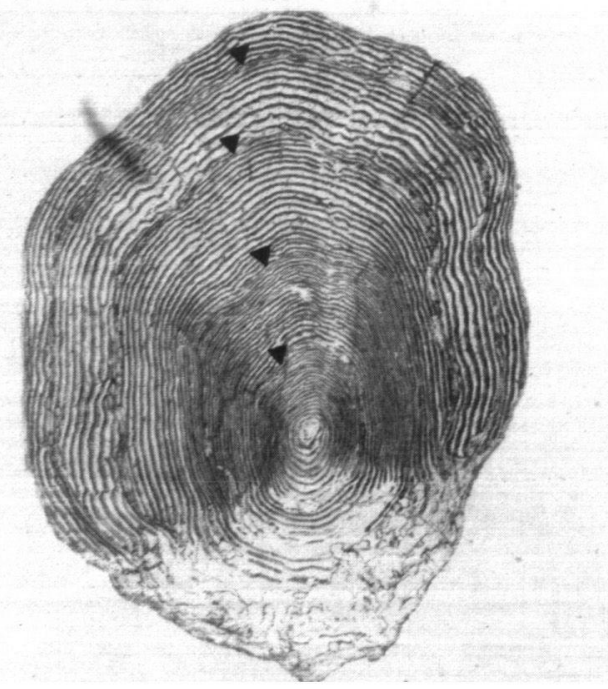

Figure 5h: Écaille de truite de mer (x 10). Calonne (Basse Normandie). Capture : 23.09.80.

LF : $552 \mathrm{~mm}$ - Age : 2+. 2avec deux fraies.

Anneau hivernal -

Figure 5h: Sea trout scale (x 10).

Calonne (Lower Normandy). Catch : 23.09.80.

FL : $552 \mathrm{~mm}$ - Age : 2.2- with two spawnings.

Winter band $\bullet$.

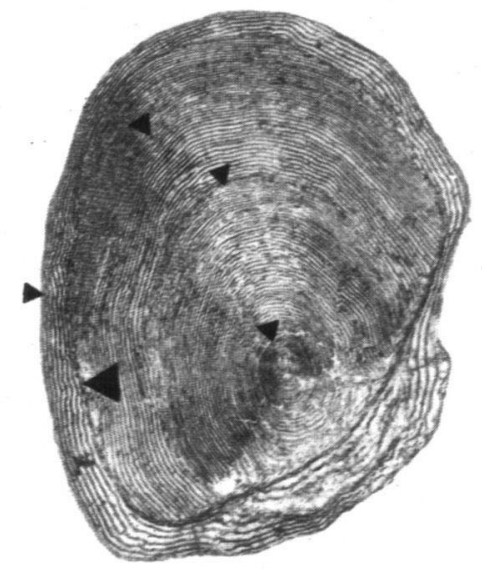

Figure 5j: Écaille de truite de mer (x 10). Orne (Basse Normandie).

LF : $710 \mathrm{~mm}$ - Age : $1+.4$ avec fraie au troisième hiver $(\downarrow)$. Chez les poissons âgés ou de grande taille la forme de l'écaille change.

Figure 5j : Sea trout scale (x 10). Orne (Lower Normandy).

FL : $710 \mathrm{~mm}$ - Age : $1+.4$ with spawning at the third sea winter (v). Scale shape of the older or large fishes changes.

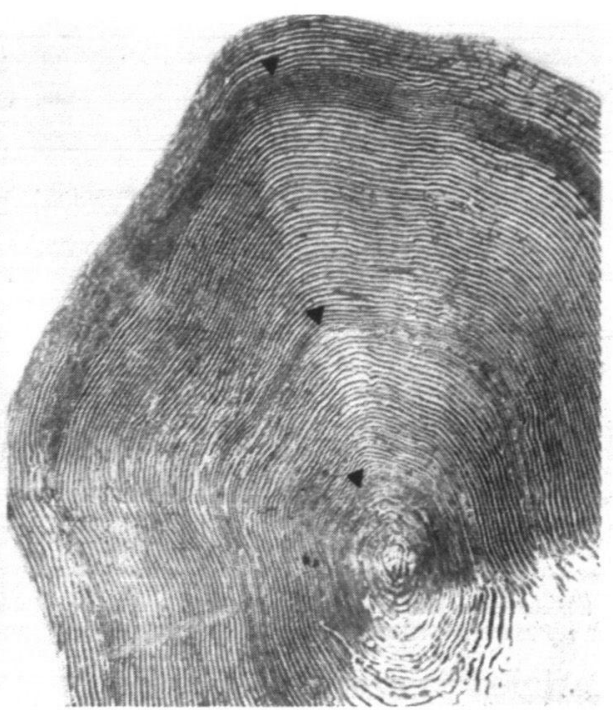

Figure 5i : Écaille de truite de mer (x 10). Orne (Basse Normandie).

LF : 705 mm - Age 1-. 2Anneau hivernal .

Figure 5i : Sea trout scale (x 10).

Orne (Lower Normandy).

FL : $705 \mathrm{~mm}$ - Age : 1* 2 *. Winter band $\bullet$.

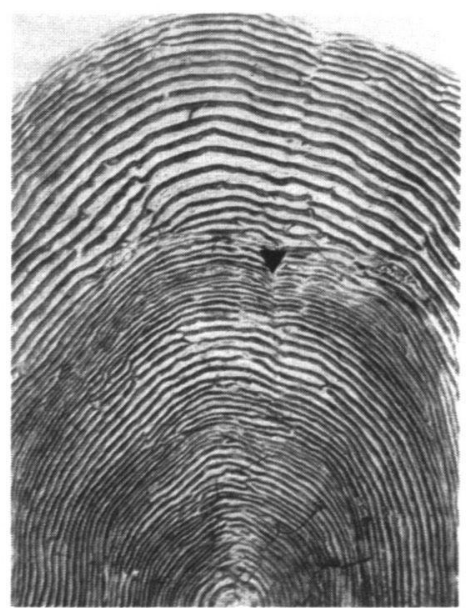

Figure 6a : Écaille de truite de mer (x 50). Calonne (Basse Normandie). Capture : 24.07.83.

LF : $368 \mathrm{~mm}$ - Age : $3+$. 0 -.

Bande hivernale en eau douce (v) Changements typiques de l'épaisseur et de l'espacement des circuli entre les phases eau douce et mer.

Figure 6a : Sea trout scale (x 50).

Calonne (Lower Normandy). Capture : 24.07.83.

FL : $368 \mathrm{~mm}$ - Age : 3-. 0 Typical winter band in freshwater $(\bullet)$ and typical changes in thickness and spacing of circuli between freshwater and sea stages. 


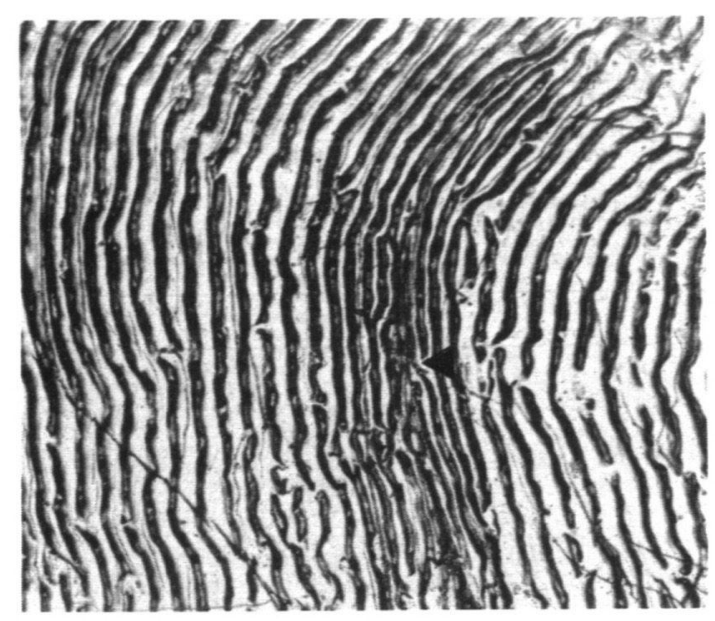

Figure 6b: Écaille de Saumon atlantique (x 50).

Anneau hivernal en mer $(\bullet)$

Figure 6b: Atlantic salmon scale (x 50). Winter band in sea $(\nu)$

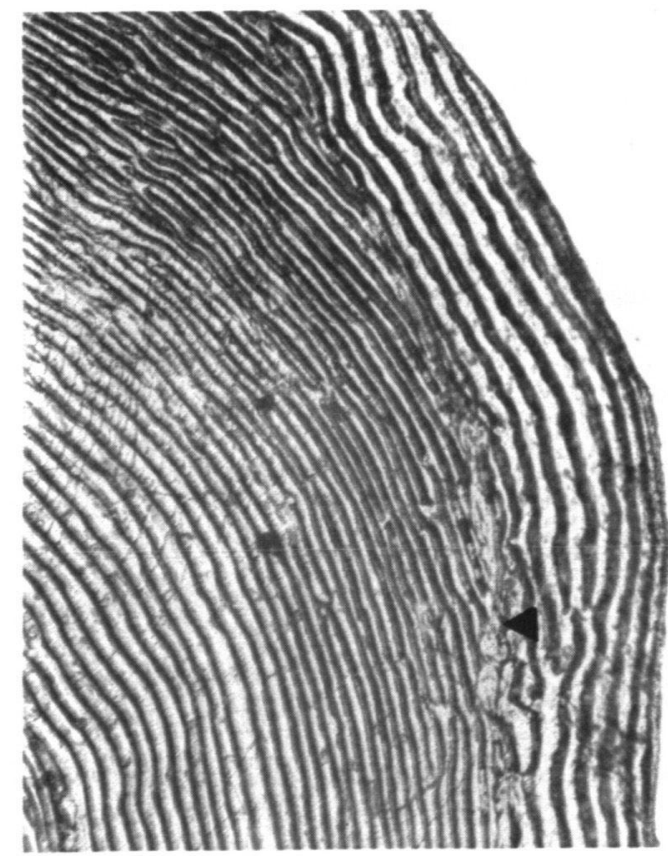

Figure 6d: Marque de fraie typique sur une écaille de truite de mer (Orne) (•) (x 50).

Figure 6d: Typical spawning mark on sea trout scale (Orne) ( $\downarrow)$ (x 50).

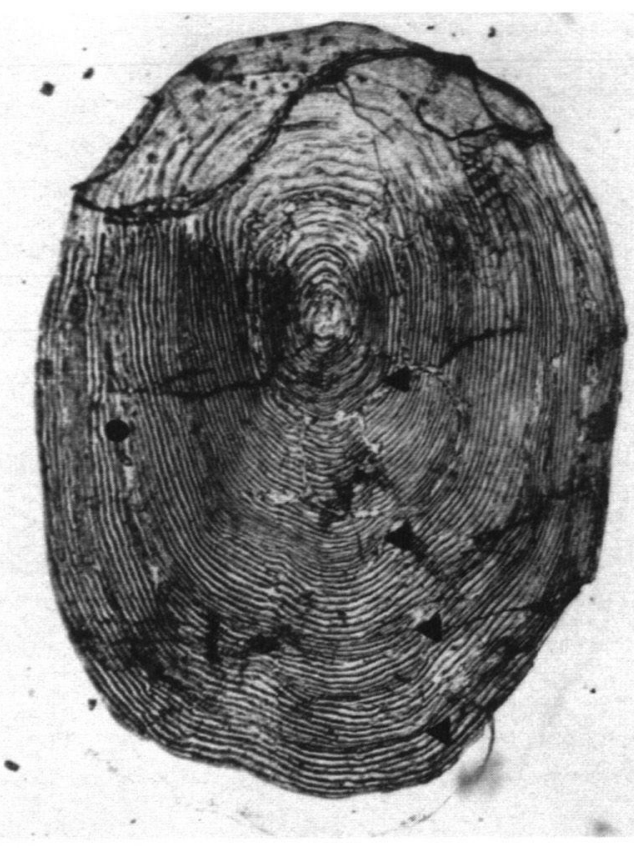

Figure 6c : Écaille de truite commune (x 30).

Scorff (Bretagne).

Capture : 18.06 .78

LF : $455 \mathrm{~mm}$ - Age : $4+$.

Anneaux hivernaux $(\nabla)$ et marques de fraie dès le troisième hiver (ब).

Figure 6c: Brown trout scale (x 30). Scorff (Brittany). Catch : 18.06.78.

FL : $455 \mathrm{~mm}$ - Age : 4 . Winter bands $(\$)$ and spawning mark from the third winter (e).

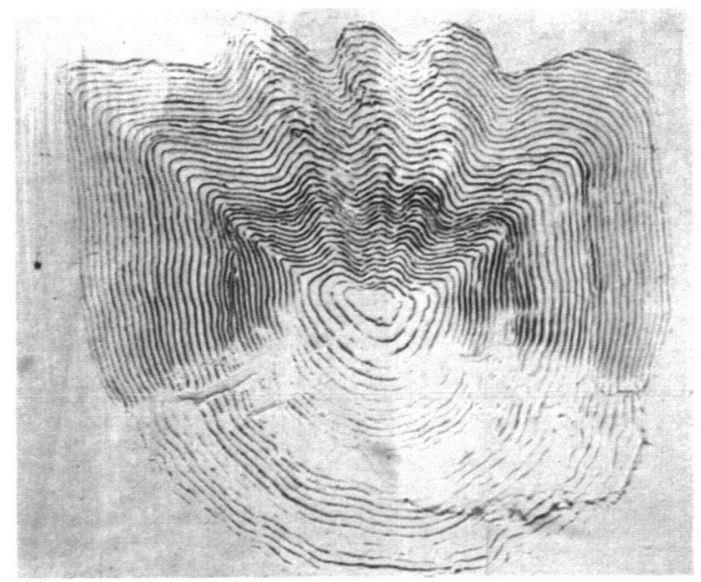

Figure 7a : Écaille d'ombre (x 30). Huisne (Mayenne). Capture : 13.09 .84$. LT : 216 mm - Age : $1+$.

Figure 7a : Grayling scale ( $x$ 30). Huisne (Mayenne). Catch : 13.09.84. TL : $216 \mathrm{~mm}$ - Age : $1+$. 


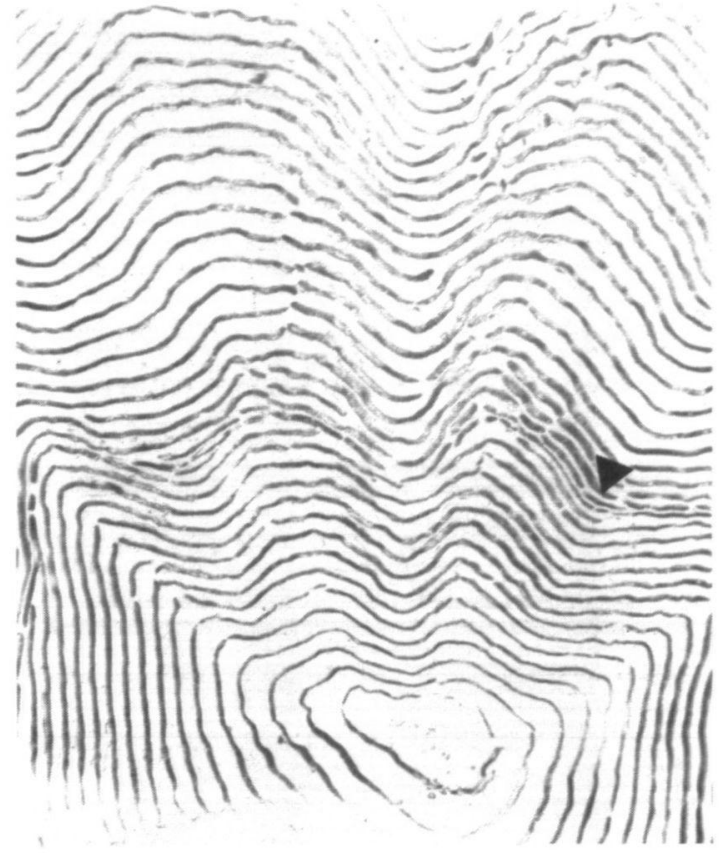

Figure 7b: Anneau hivernal sur une écaille d'ombre (x 50) ( $)$.

Figure 7b: Winter band on grayling scale (x 50) (v).

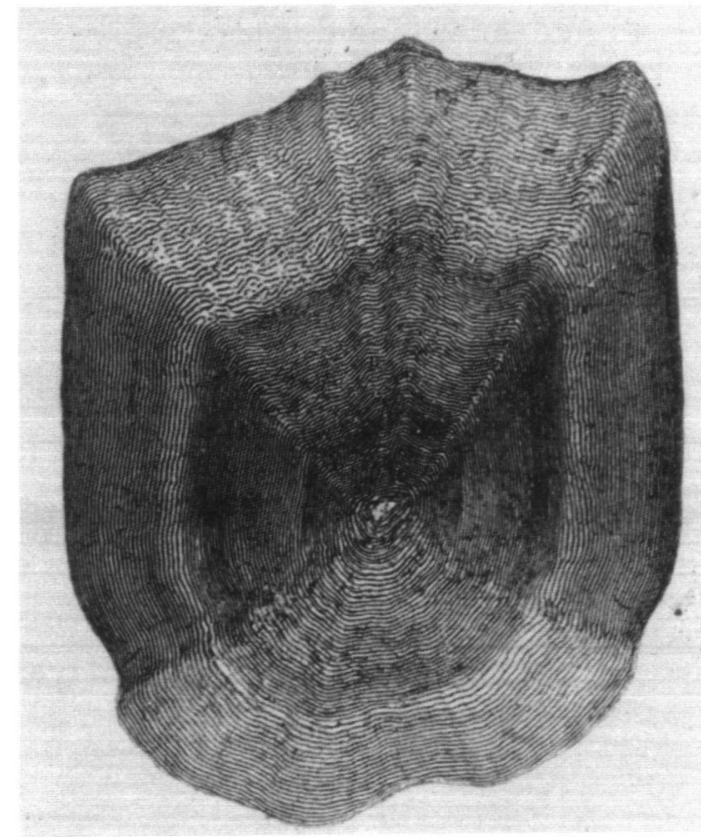

Figure 8a : Écaille de corégone (x 10). Lac Léman.

Capture : 12.82 .

LT : 395 mm - of-Age : $2+$.

Figure 8a: White fish scale $(x 10)$.

Lac Leman.

Catch : 12.82 .

TL : $395 \mathrm{~mm}$ - $\delta$ - Age : $2+$.

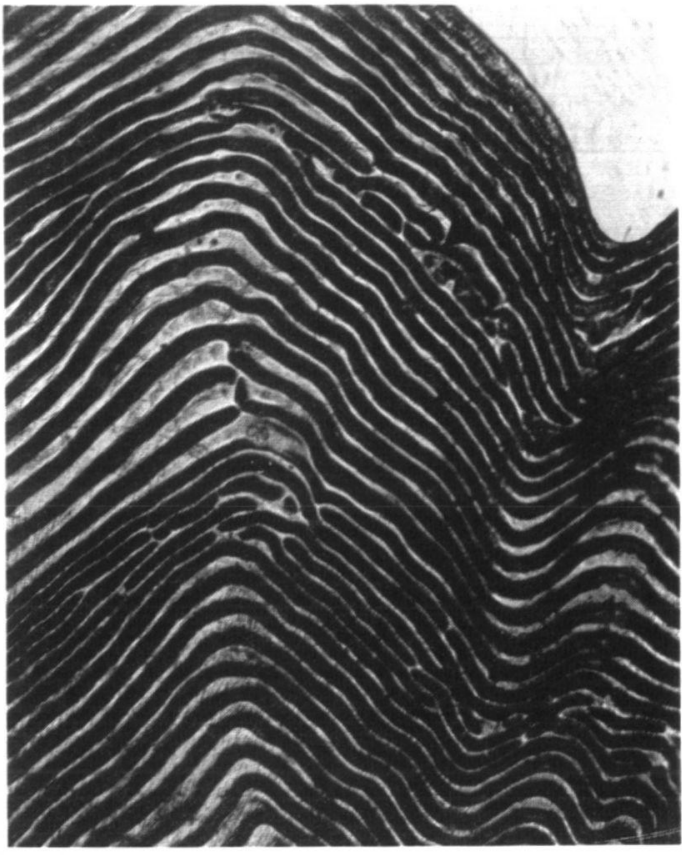

Figure 7c : Écaille d'ombre (x 50) Élevage d'Augerolles (Massif Central). Capture : 28.04.86.

LF : $300 \mathrm{~mm}$ - $Q$ - Age : 3+. Anneau de ponte possible à 3 ans $(\bullet)$.

Figure 7c: Grayling scale (x 50). Hatchery of Augerolles (Massif Central). Catch : 28.04.86.

FL : $300 \mathrm{~mm}$ - $\$$ - Age : $3+$.

Possible spawning mark at 3 years old $(\bullet)$.

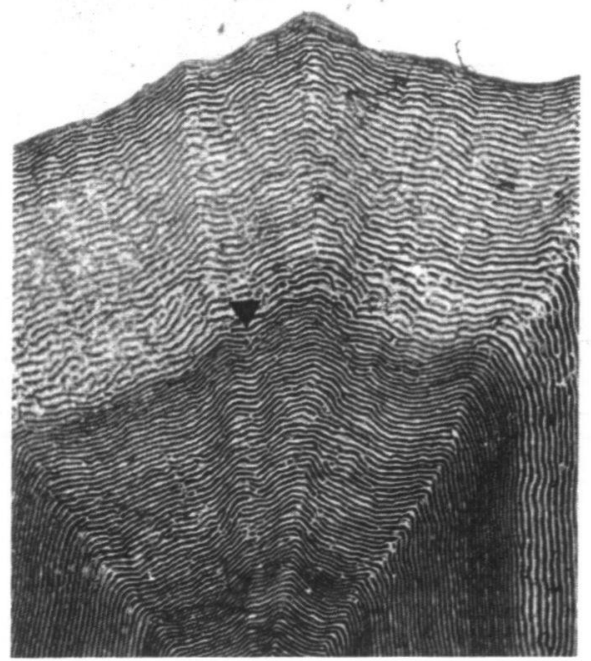

Figure 8b : Anneau hivernal sur une écaille de corégone $(\bullet)(\times 50)$.

Age : $2+$.

Figure $8 \mathrm{~b}$ : Winter band on white fish scale (•) (x 50).

Age : $2+$. 


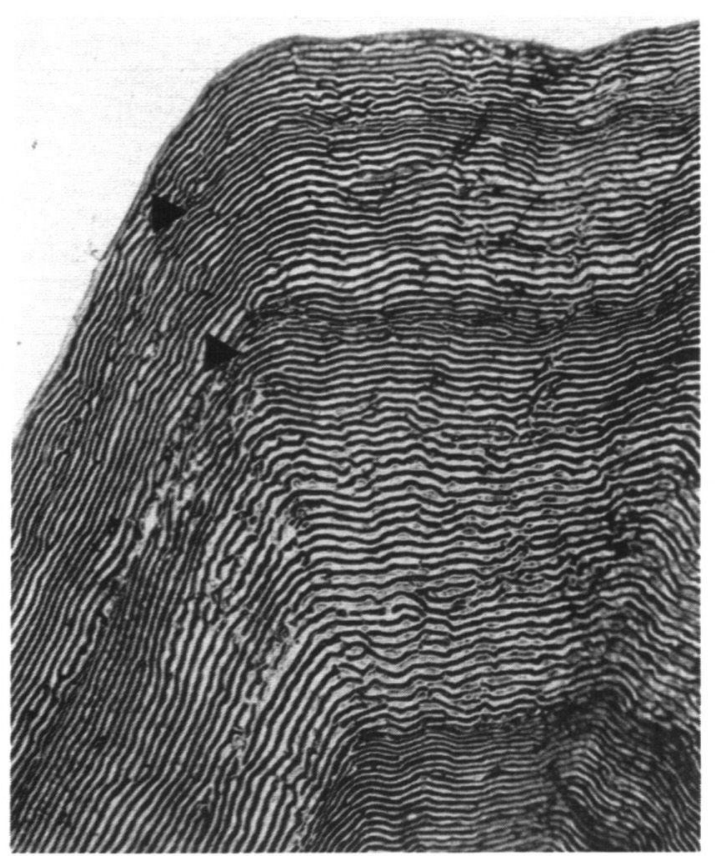

Figure 8c : Écaille de Corégone (x 50). Lac Léman.

Capture : 12.82 .

LT : $470 \mathrm{~mm}$ - $q$.

Age : $3+$ annuli caractéristiques ou possibles marques de fraie (•).

Figure 8c: White fish scale (x 50).

Lac Leman.

Catch : 12.82 .

TL : $470 \mathrm{~mm}$ - $Q$.

Age : 3, typical winter bands or possible spawning marks ( () ).

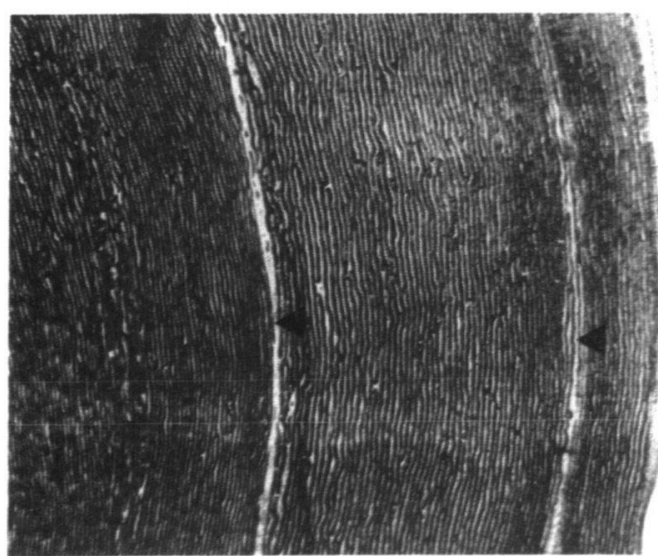

Figure 9b: Anneaux hivernaux ( $(5)$ sur une écaille de brochet $(x 50)$.

Boutonne (Charente).

Capture : 15.08.83.

LF : $405 \mathrm{~mm}$ - - Age : 3 t.

Figure 9b: Winter bands (D) on pike scale (x 50).

Boutonne (Charente).

Catch : 15.08.83.

FL : $405 \mathrm{~mm}$ - $Q$ - Age : 3 t.

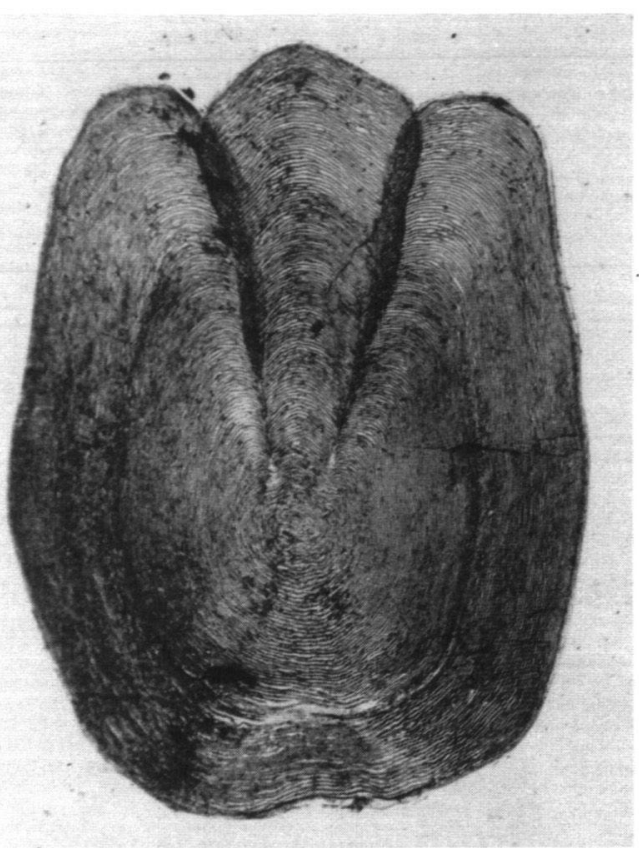

Figure 9a : Écaille de brochet (x 30).

Boutonne (Charente).

Capture : 18.08.84.

LF : 215 mm - of - Age : $1+$.

Figure 9a: Pike scale (x 30).

Boutonne (Charente).

Catch : 18.08.84.

FL : $215 \mathrm{~mm}-\not \hat{~-~ A g e ~: ~} 1+$.

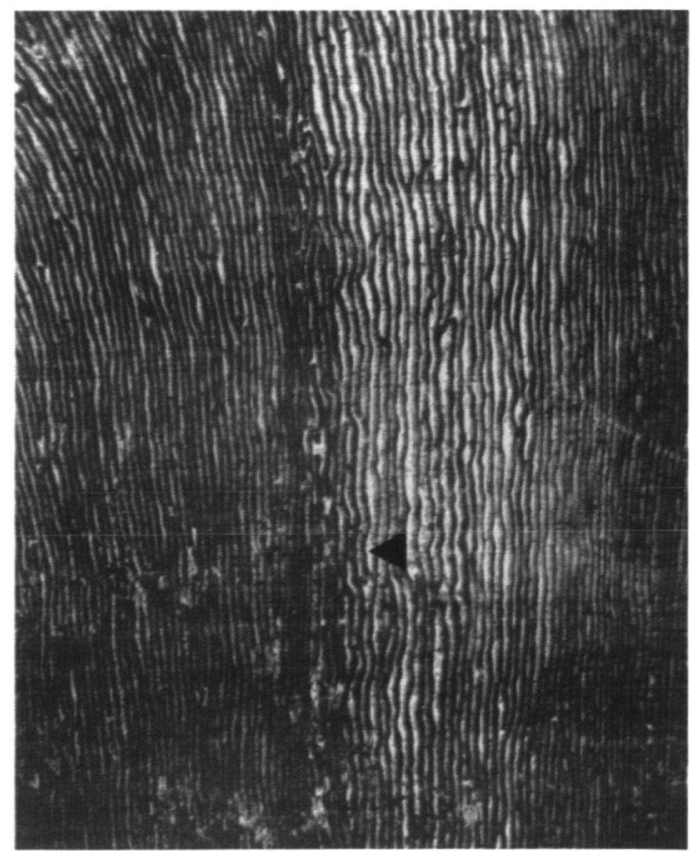

Figure 9c: Structure d'un anneau hivernal sur une écaille de brochet en étang $(\bullet)(x$ 100).

Figure 9c: Structure of winter band on pike scale in pond $(\nu)(x 100)$. 


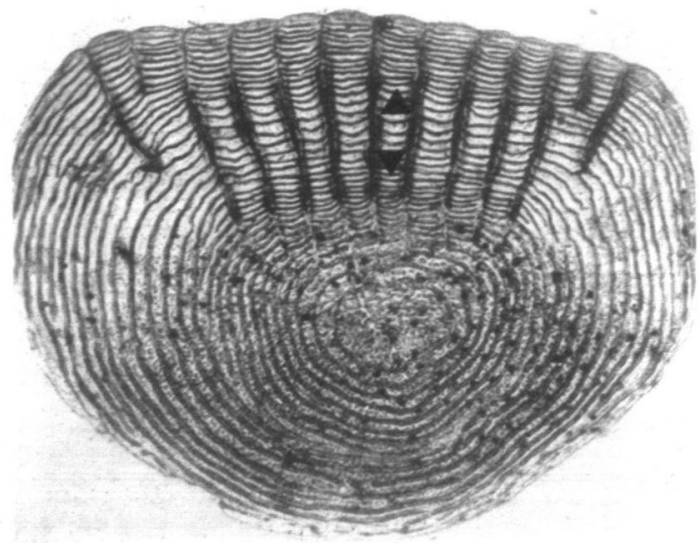

Figure 10 : Écaille de Gambusie (x 100). Canal de Brouage (Charente). Capture : 27.03.86. LF : $55 \mathrm{~mm}$ - ? .

Age : difficile à déterminer. Changements dans la vitesse de croissance ( $\bullet$ ).

Figure 10: Gambusia scale (x 100).

Brouage Canal (Charente). Catch : 27.03.86.

FL : $55 \mathrm{~mm}$ - 9

Age : hard to determine.

Changes in growth speed $(\bullet)$.

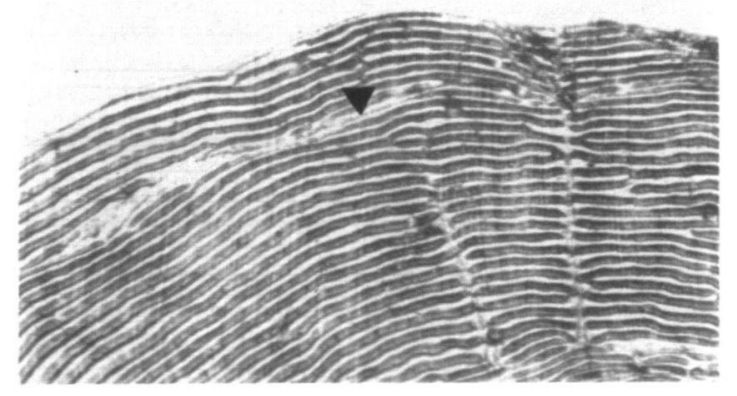

Figure 11b: Anneau hivernal sur une écaille d'ablette $(\bullet)$ (x 100).

Figure 11b: Winter band on bleak scale (D) $(\times 100)$.

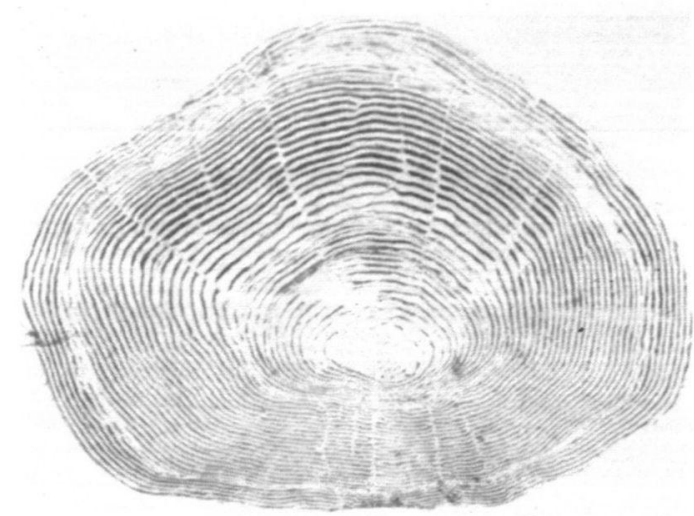

Figure 11a : Écaille d'ablette $(\times 30)$. Le Meu (Bretagne).

Capture : 27.11.84.

LF : $103 \mathrm{~mm}$ - Age : 2 †.

Figure 11a: Bleak scale (x 30).

Le Meu (Brittany).

Catch : 27.11.84.

FL : $103 \mathbf{m m}-$ Age : 2 t.

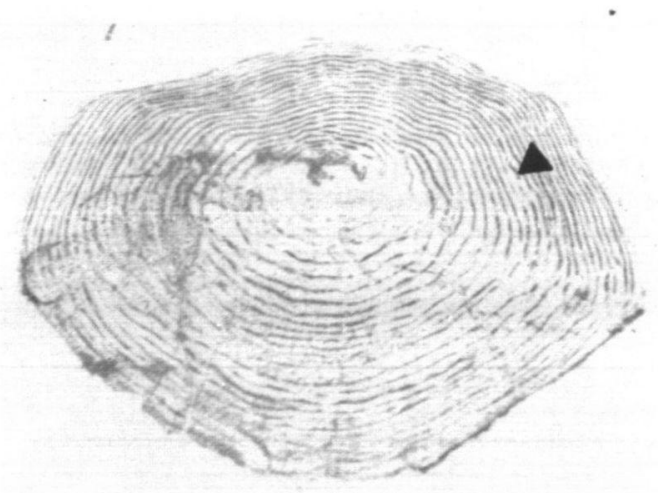

Figure 12 : Écaille d'able $(\times 30)$ Le Meu (Bretagne).

Capture : 3.10.83.

LF : 70 mm - Age : 1+. Anneau hivernal $\bullet$.

Figure 12 : Rain bleak scale (x 30). Le Meu (Brittany).

Catch : 3.10.83.

FL : $70 \mathrm{~mm}$ - Age : 1 Winter band $\bullet$. 


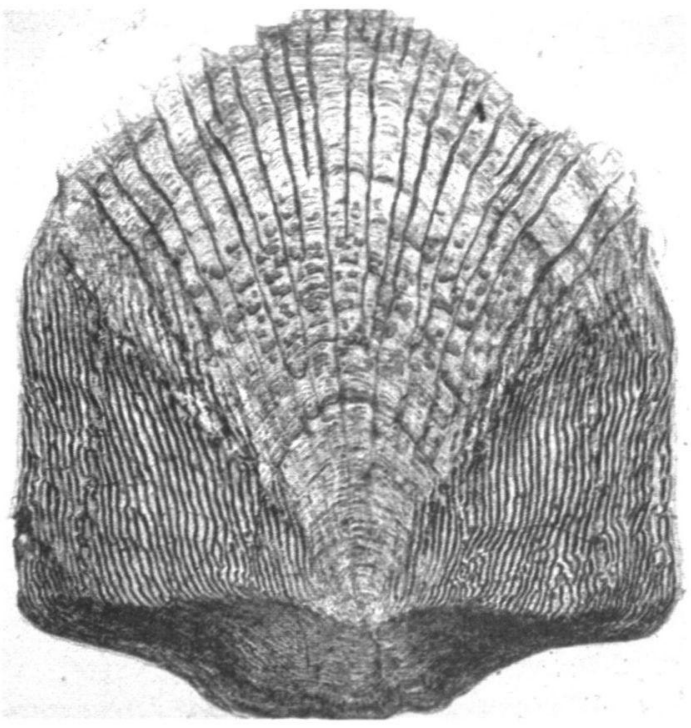

Figure 13a : Écaille de Goujon (x 20). La Flume (Bretagne). Capture : 26.08.80. LF : 137 mm - Age : 3 †.

Figure 13a: Gudgeon scale ( $\times 20)$. La Flume (Brittany). Catch : 26.08.80. FL : $137 \mathrm{~mm}$ - Age : 3 †.

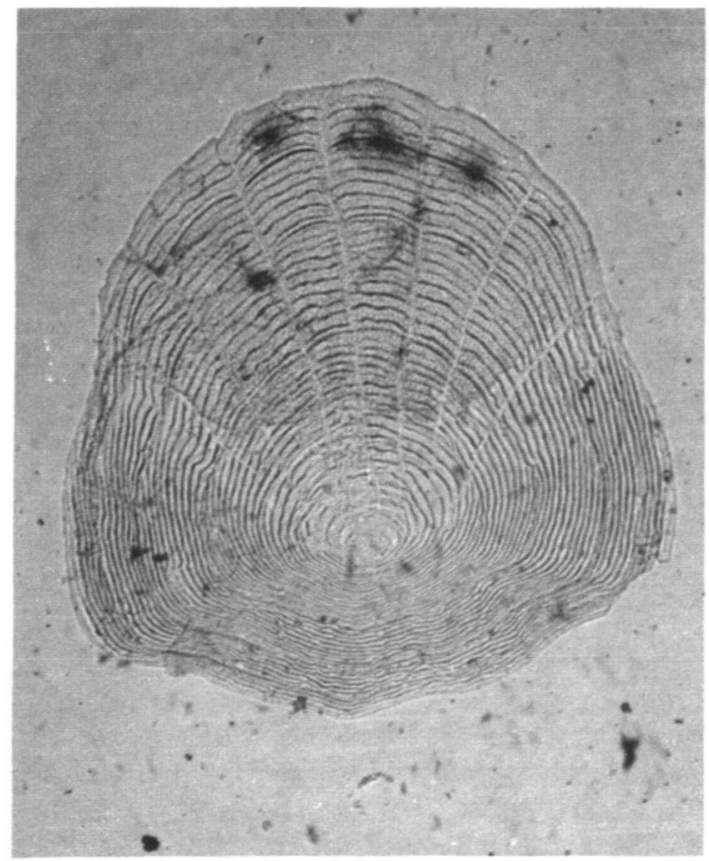

Figure 14a: Écaille d'ablette spirlin (x 20). Colmont (Mayenne).

Capture : 12.10 .80 .

LF : $100 \mathrm{~mm}$ - Age : 2 +.

Figure 14a: Stream bleak scale (x 20). Colmont (Mayenne).

Catch : 12.10.80.

FL : $100 \mathrm{~mm}$ - Age : 2 . .

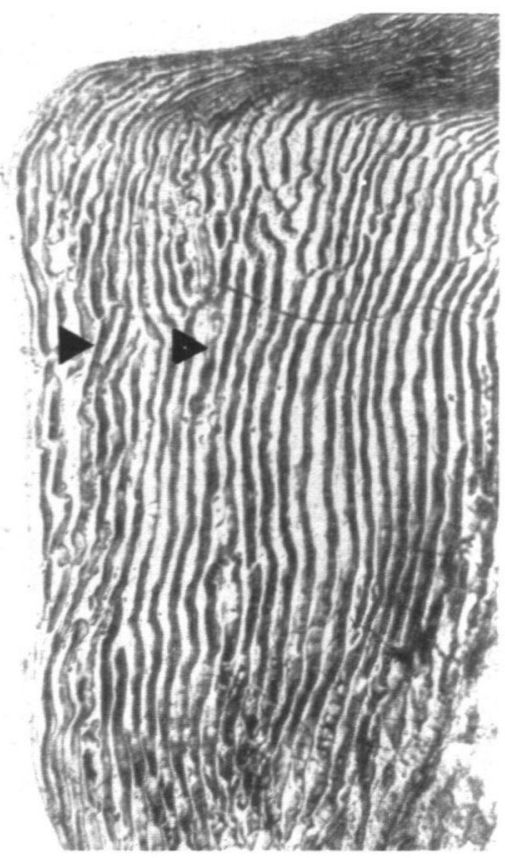

Figure 13b: Anneaux hivernaux $(\triangleright)$ sur une écaille de goujon ( $x$ 50).

Figure 13b: Winter bands $(\omega)$ on gudgeon scale $(x 50)$.

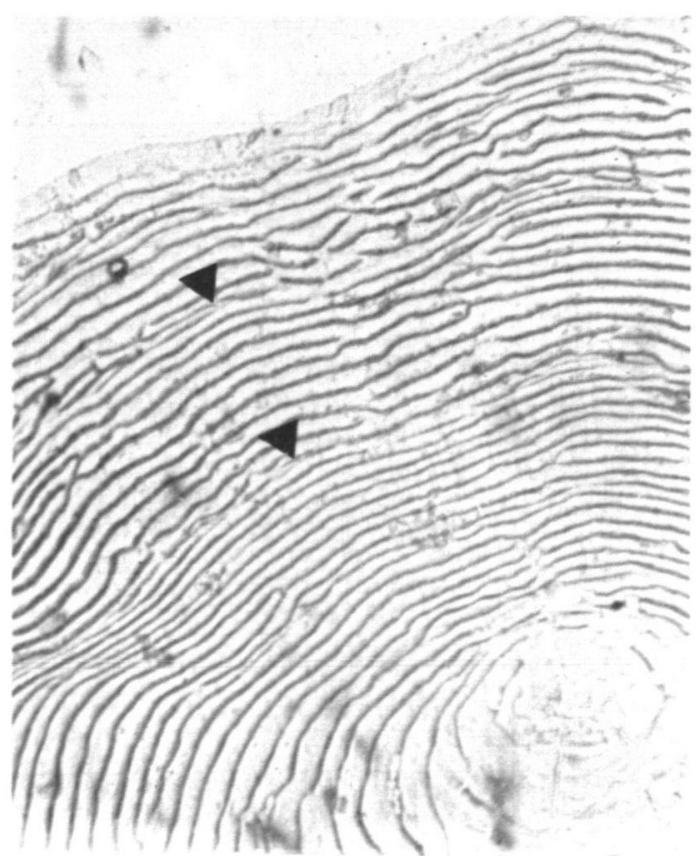

Figure 14b: Anneaux hivernaux sur une écaille d'ablette spirlin (D) (x 100).

Figure 14b: Winter bands on stream bleak scale $(\bullet)(x 100)$. 


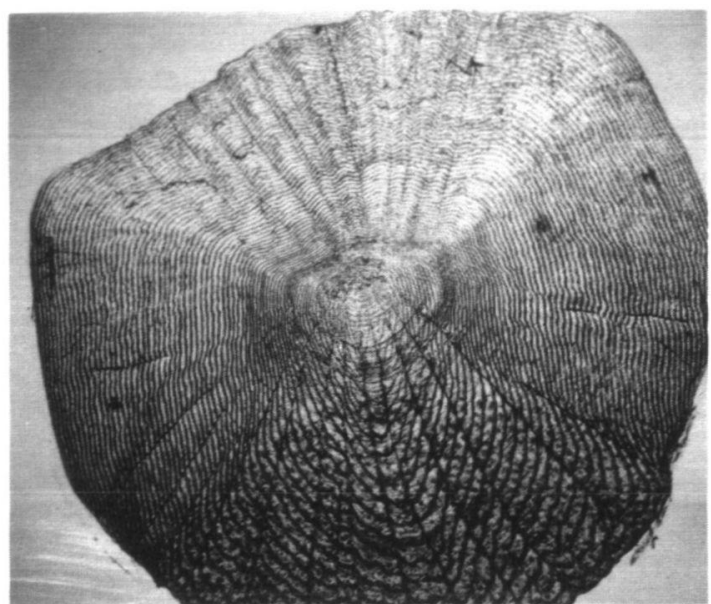

Figure 15a: Ecaille de carpe herbivore (x 10).

Pisciculture de Lorraine.

Capture : 10.01.87.

LF : $228 \mathrm{~mm}$ - Age : 1 t.

Figure 15a: Herbivor carp scale (x 10).

Hatchery of Lorraine.

Catch : 10.01.87.

FL : $228 \mathrm{~mm}$ - Age : $1 \mathrm{t}$.

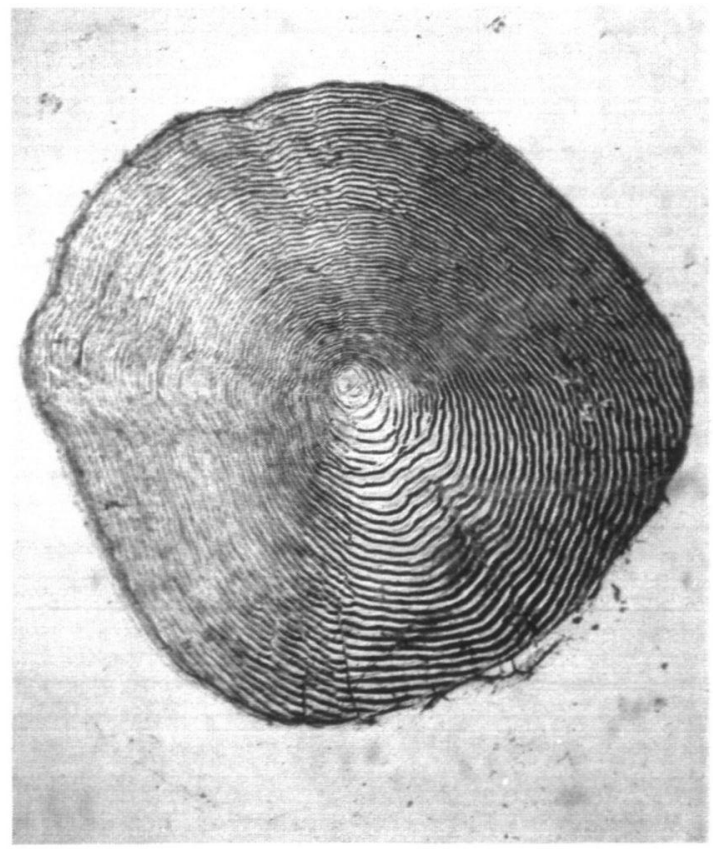

Figure 16a: Écaille de carpe argentée (x 20).

Pisciculture de Lorraine.

Capture : 22.01.87.

LF : $318 \mathrm{~mm}$ - Age : 1 †.

Figure 16a : Silver carp scale (x 20).

Hatchery of Lorraine.

Catch : 22.01.87.

LF : 318 mm - Age : 1 †.

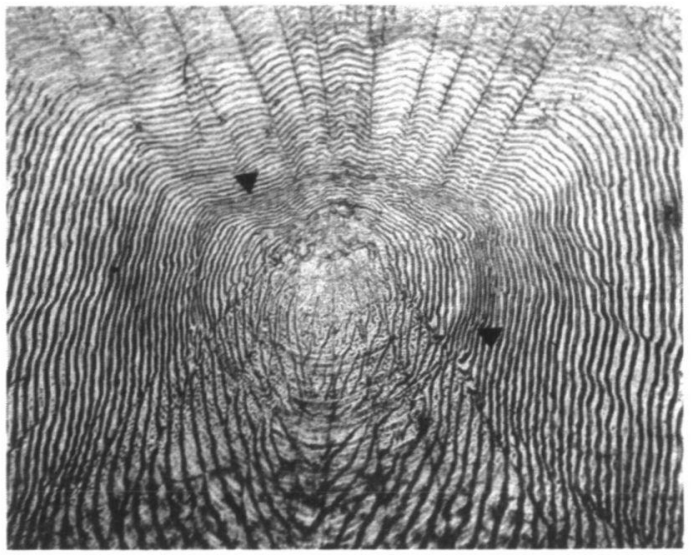

Figure 15b: Anneau hivernal sur une écaille de carpe herbivore ( $)$ (x 30).

Figure 15b : Winter band $(\downarrow)$ on herbivorous carp scale (x 30).

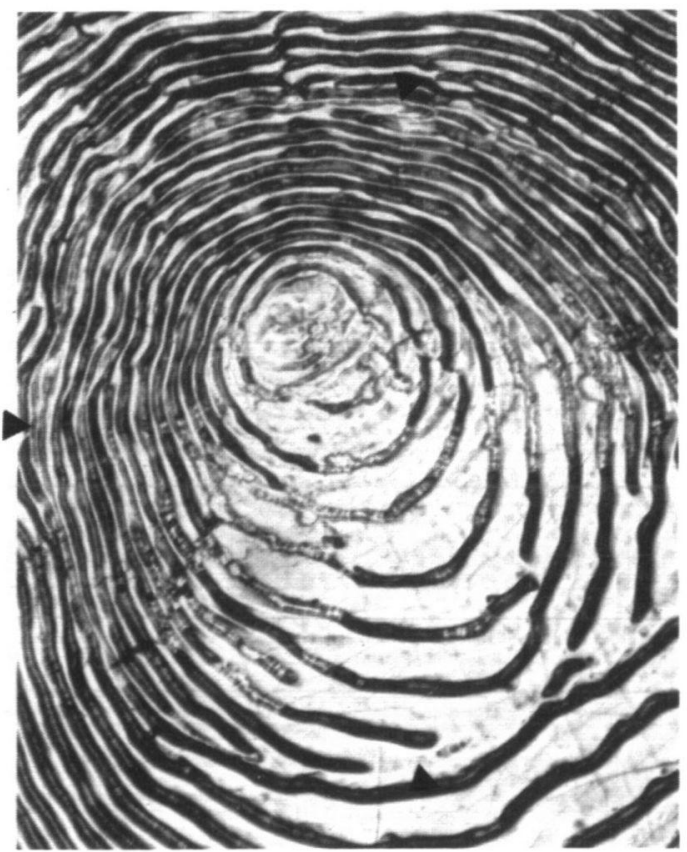

Figure 16b: Anneau hivernal sur une écaille de carpe argentée $(\bullet)$ (x 100).

Figure 16b: Winter band on silver carp scale $(\bullet)(x 100)$. 


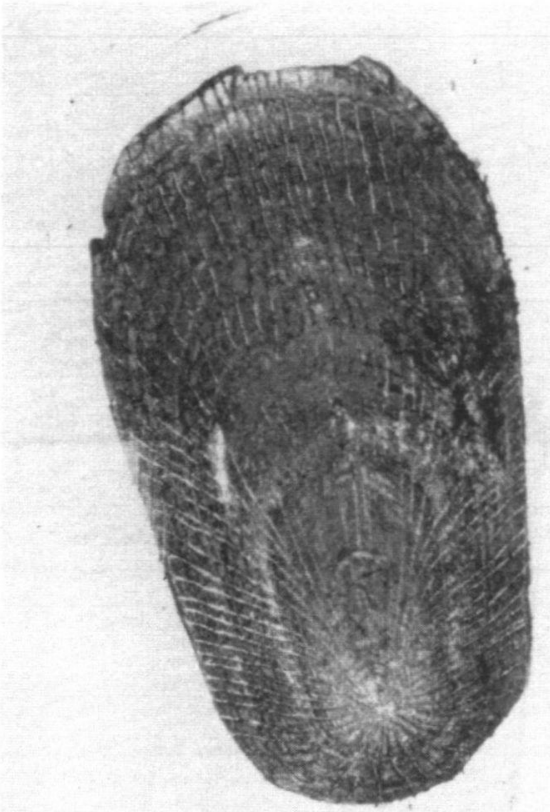

Figure 17a : Écaille de tanche $(\times 10)$. Étang de Pacé (Bretagne). Capture : 10.09.81. LF : 316 mm - ? Age possible : $5+$ ?

Figure 17a: Tench scale $(\times 10)$ Pacé Pond (Brittany). Catch : 10.09.81. FL : $316 \mathrm{~mm}-\mathrm{q}$ Possible age : $5 i$ ?

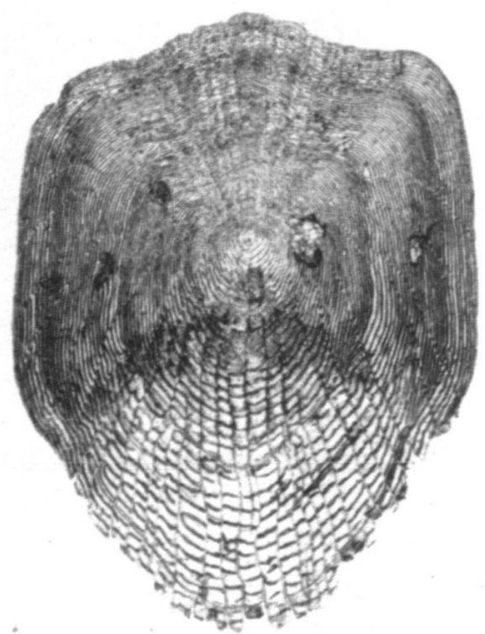

Figure 18a : Écaille de barbeau commun (x 10)

Étang de Rannée (Bretagne). Capture : 5.04 .78 .

LF : 275 mm - Age : 2-.

Figure 18a : Common barbel scale (x 10). Rannée Pond (Brittany).

Catch : 5.04.78.

FL : 275 mm - Age : 2+.

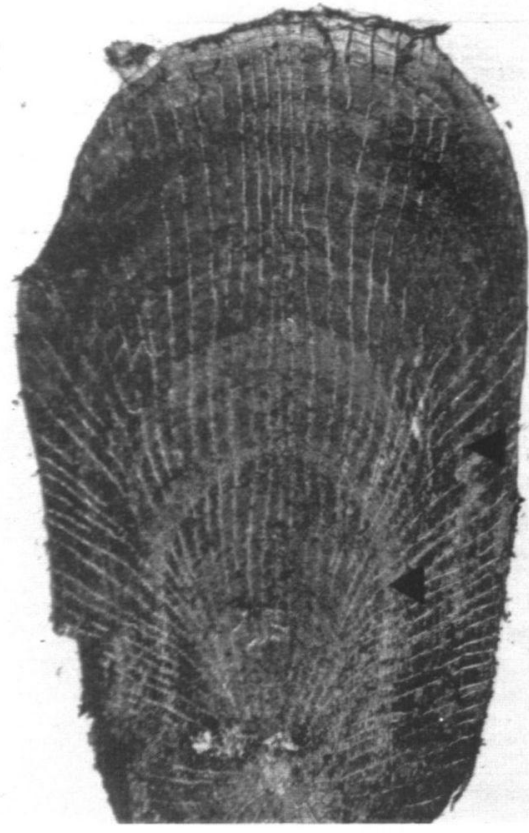

Figure 17b: Arrêts hivernaux $(D)$ sur une écaille de tanche $(x 20)$.

Figure 17b : Winter bands $(\omega)$ on tench scale $(x 20)$.

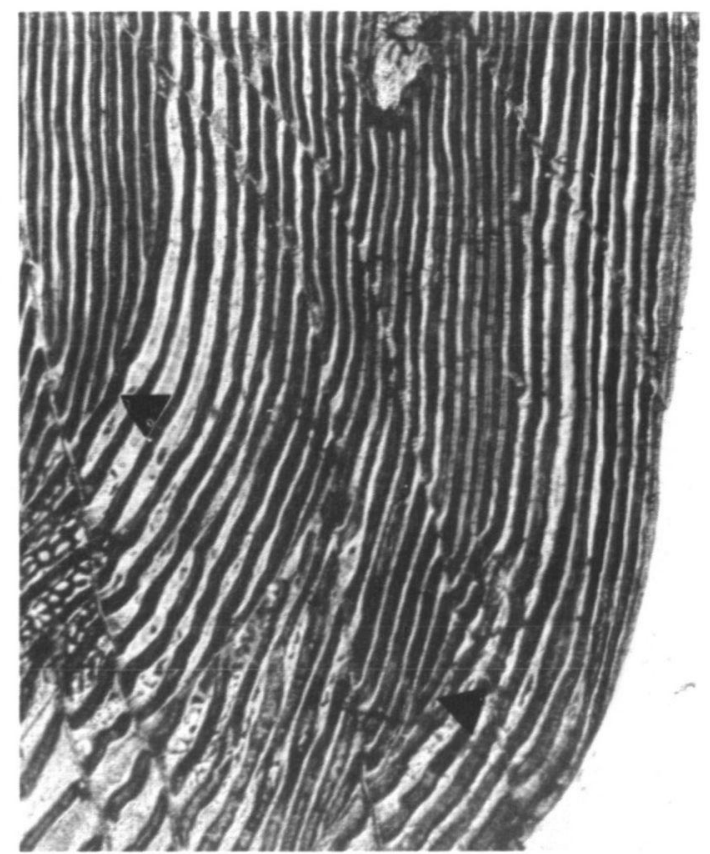

Figure 18b : Anneaux hivernaux sur une écaille de barbeau commun (x 50) (D).

Figure 18b : Winter bandson common barbel scale (x 50) ( $)$. 


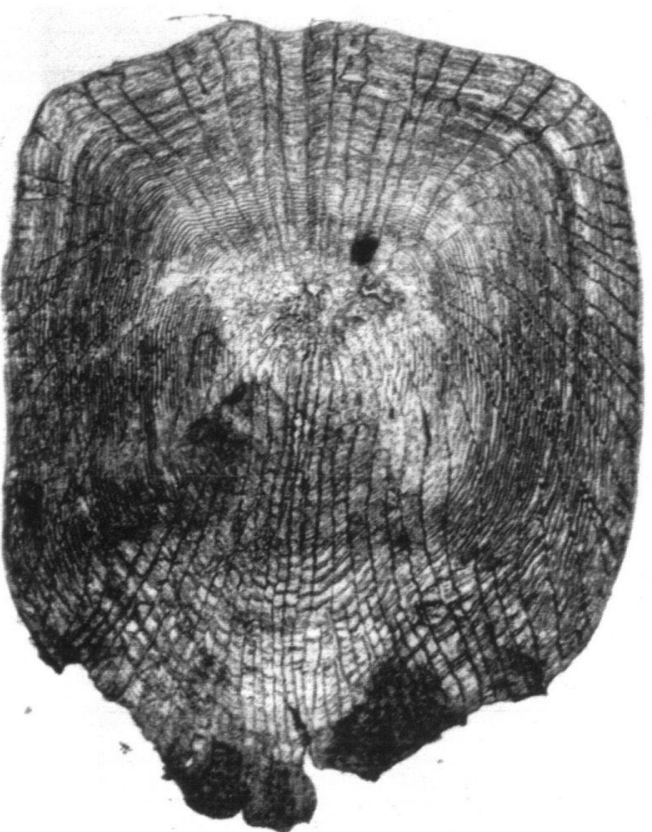

Figure 19 : Écaille de barbeau méridional (x 10).

Herbasse (Rhône).

LF : $200 \mathrm{~mm}$.

Age possible : $3+$ ?

Figure 19 : Meridional barbel scale (x 10). Herbasse (Rhône).

FL : $200 \mathrm{~mm}$.

Possible age : $3 \nmid$ ?

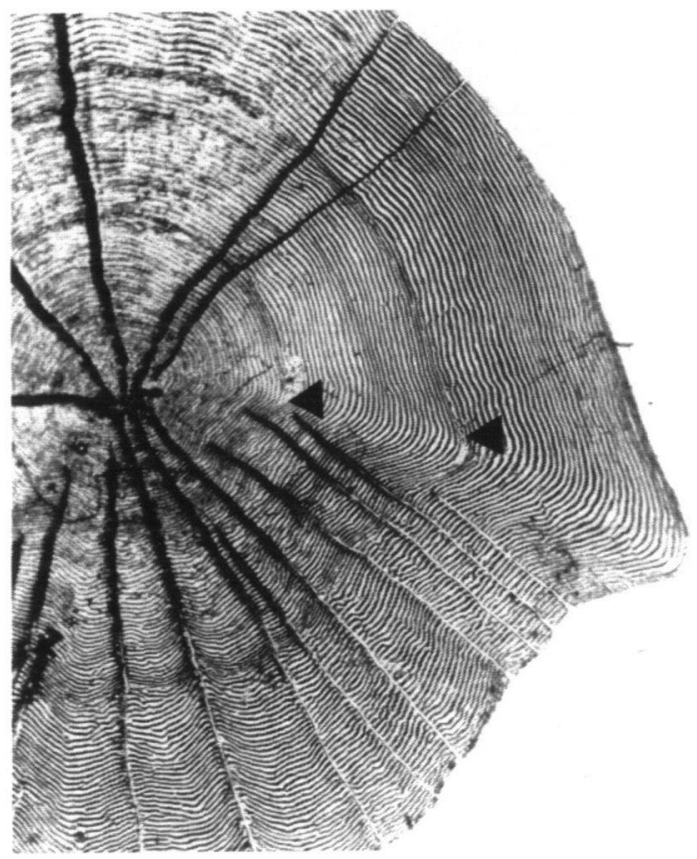

Figure 20b: Bandes hivernales ( $)$ sur une écaille de gardon de $2+$ ans (x 20).

Figure $20 b$ : Winter bands $(\bullet)$ on $2+$ years old roach scale $(\times 20)$.

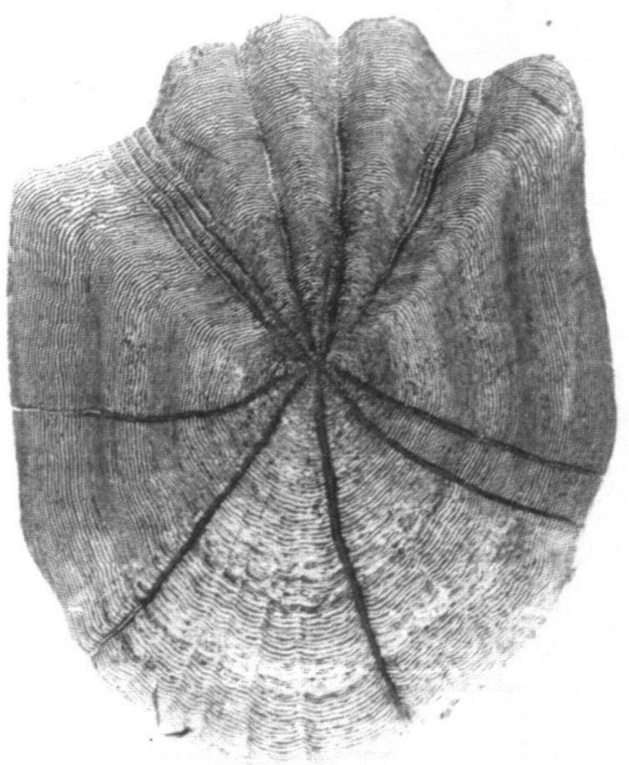

Figure 20a : Écaille de gardon (x 10). Lac de Guerlédan (Bretagne). Capture : 14.05.81.

LF : $160 \mathrm{~mm}-$ Age : 3 t.

Figure 20a: Roach scale (x 10). Guerledan Lake (Brittany). Catch : 14.05.81. FL : $160 \mathrm{~mm}$ - Age : 3 †.

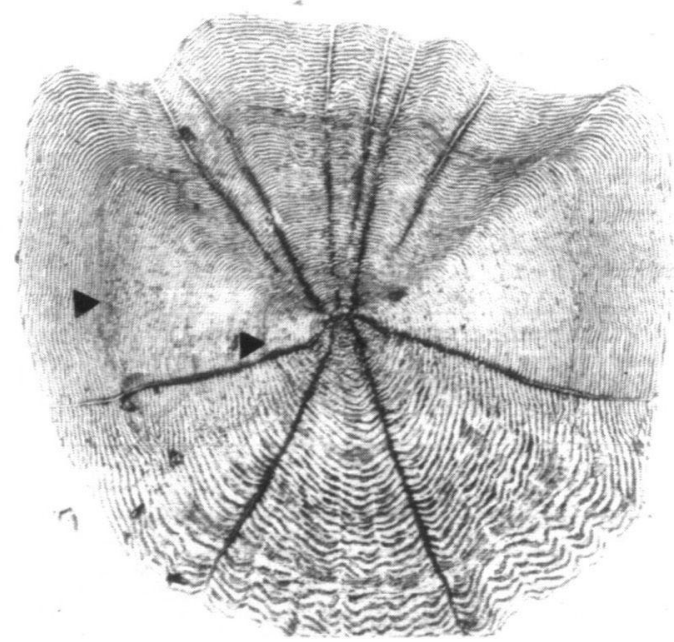

Figure 21 : Ecaille de rotengle (x 10). Étang de Chavagne (Bretagne). Capture : 1.10 .81 .

LF : $104 \mathrm{~mm}$ - Age : 2 Anneau hivernal $\bullet$.

Figure 21 : Rudd scale (x 10). Chavagne Pond (Brittany). Catch : 1.10.81. FL : $104 \mathrm{~mm}$ - Age : $2+$. Winter band $\bullet$. 


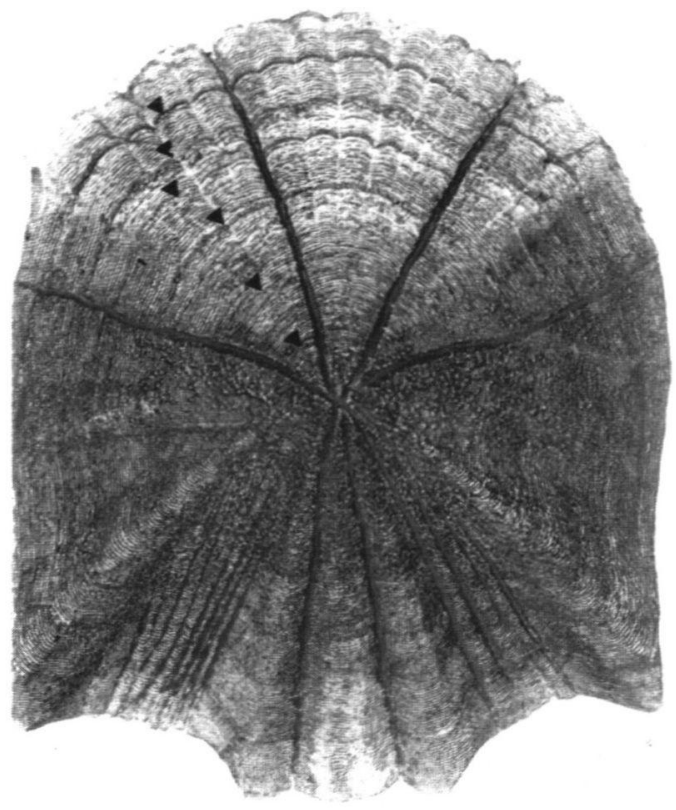

Figure 22 : Écaille d'Hybride gardon-rotengle $(x$ 10).

Étang de Pacé (Bretagne).

Capture : 30.10 .85 .

LF : $220 \mathrm{~mm}$ - Age : $6+$.

Anneau hivernal $\bullet$.

Figure 22 : Roach-Rudd hybrid scale (x 10). Pacé Pond (Brittany).

Catch : 30.10.85.

FL : $220 \mathrm{~mm}$ - Age : 6+. Winter band.

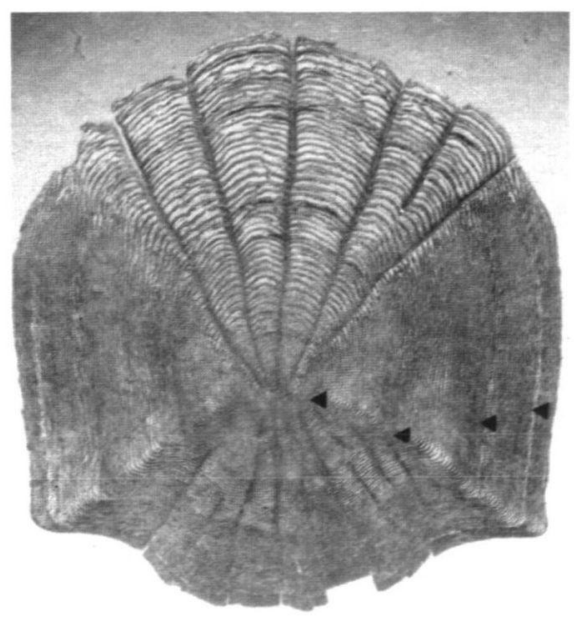

Figure 24 : Écaille de Soffie.

Tarn (Tarn) (x 10).

Capture : 11.05 .86 .

LF : $250 \mathrm{~mm}$ - Age : 4 -.

Anneau hivernal .

Figure 24 : South west-european nose.

Tarn (Tarn) (x 10).

Catch : 11.05.86.

FL : $250 \mathrm{~mm}$ - Age : 4 -.

Winter band $\bullet$.

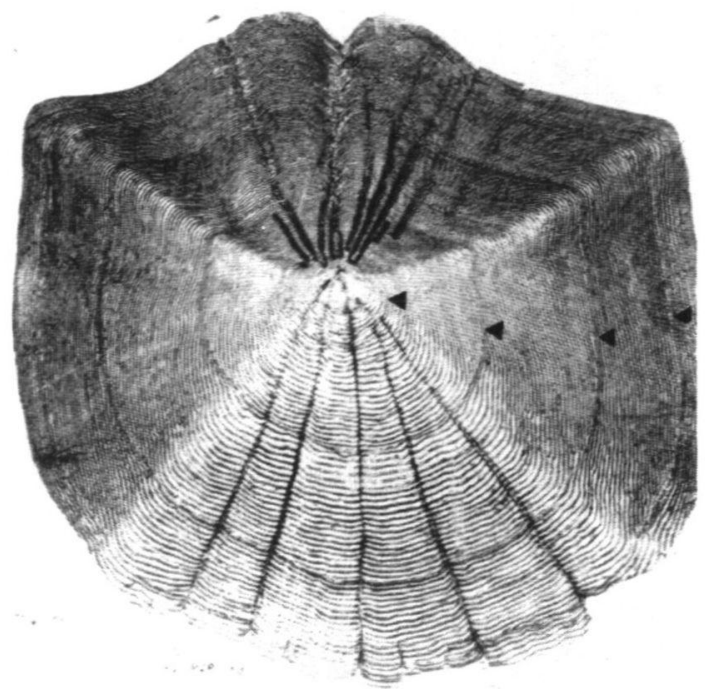

Figure 23 : Écaille de hotu (x 10).

Saône (Rhône).

LF : $\mathbf{3 3 0} \mathbf{~ m m}$ - Age : $4+$.

Anneau hivernal $\bullet$.

Figure 23 : Common nose scale (x 10). Saône (Rhône).

$F L: 330 \mathrm{~mm}$ - Age : $4+$. Winter band $\bullet$.

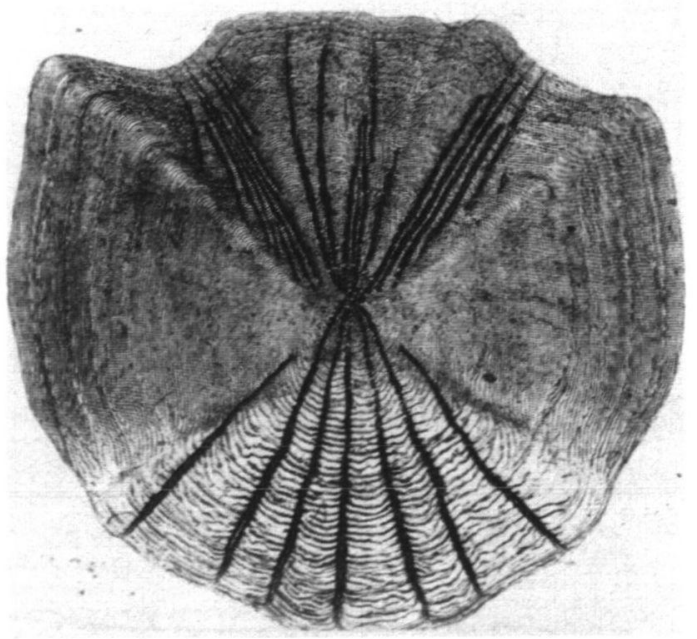

Figure 25a : Écaille de vandoise (x 10). Scorff (Bretagne). Capture : 23.09.82

LF : $264 \mathrm{~mm}$ - Age : $5+$.

Figure 25a : Dace scale (x 10).

Scorff (Brittany).

Catch : 23.09.82.

FL : $264 \mathrm{~mm}$ - Age : 5 t. 


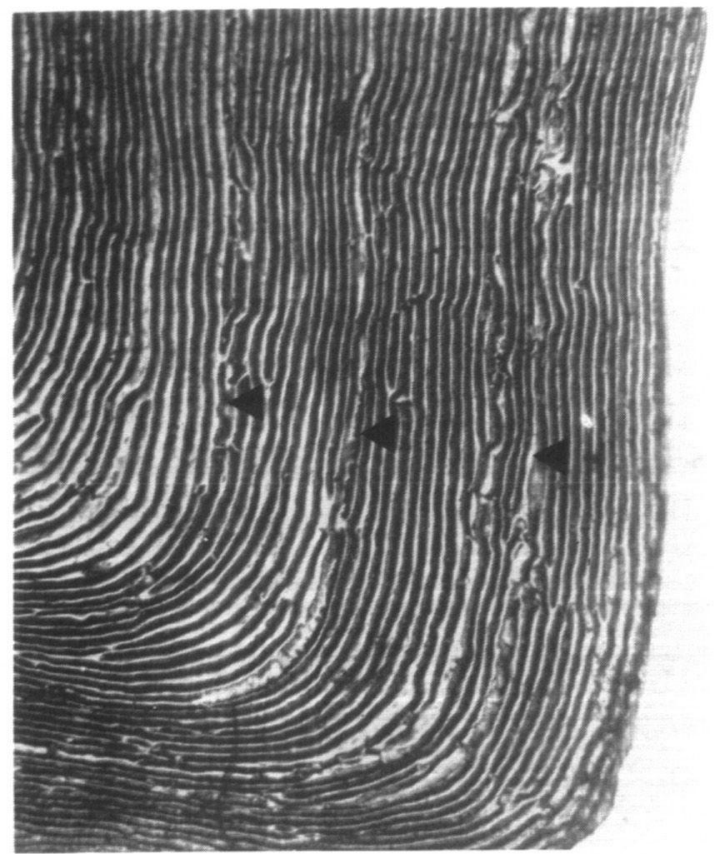

Figure 25b : Bandes hivernales $(\nu)$ sur une écaille de Vandoise ( $x$ 50).

Figure 25b : Winter bands $(*)$ on dace scale (x 50).

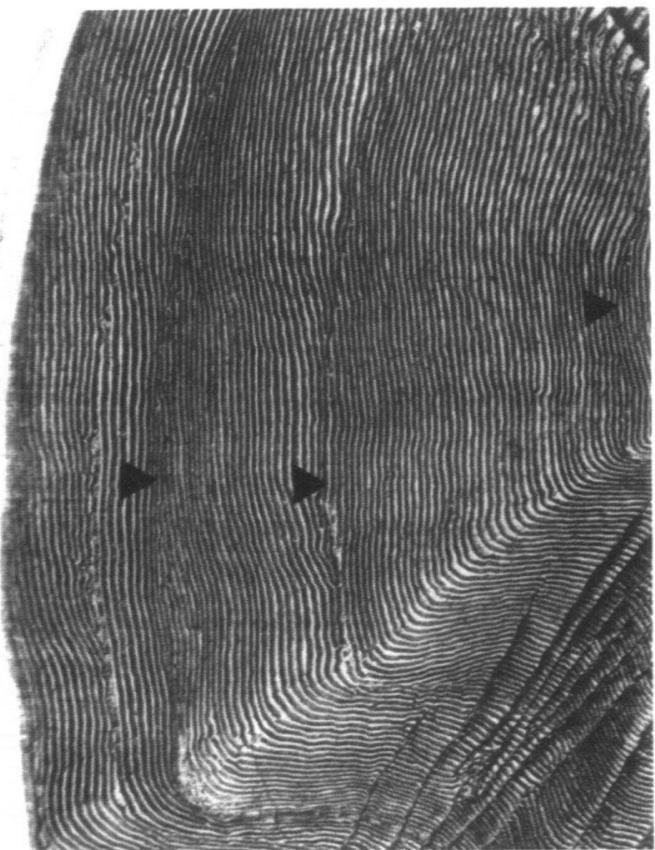

Figure 26b : Bandes hivernales ( $)$ sur une écaille de chevaine de 4+ (x 50).

Figure 26b : Winter bands $(*)$ on $4-$ years old chub scale (x 50).

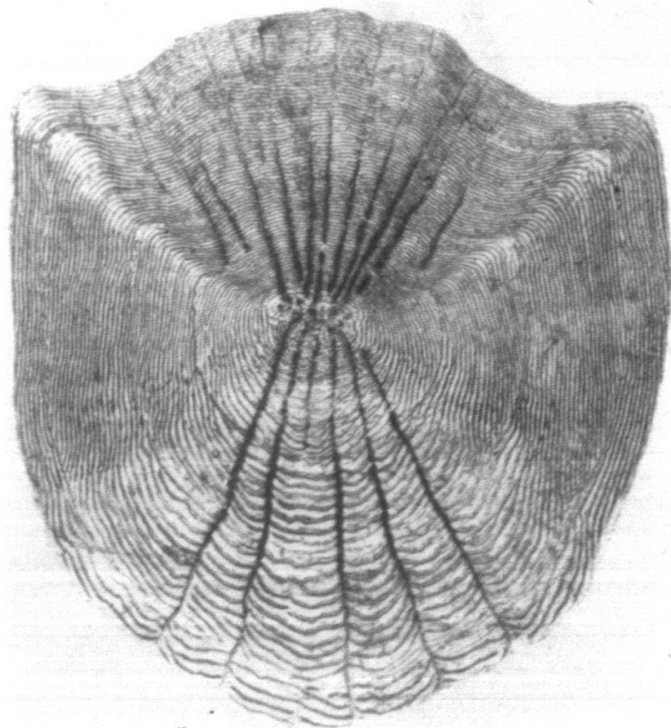

Figure 26a : Écaille de chevaine (x 10). Colmont (Mayenne).

Capture : 12.10.80.

LF : $158 \mathrm{~mm}$ - Age : 4 t.

Figure 26a : Chub scale (x 10).

Colmont (Mayenne).

Catch : 12.10.80.

FL: $158 \mathrm{~mm}$-Age : 4 t.

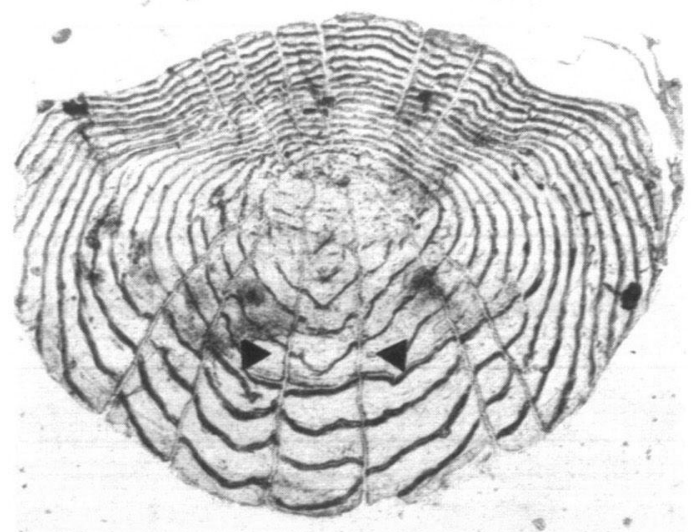

Figure 26c : Ecaille de chevaine (x 50). Flume (Bretagne). Capture : 3.07.86.

LF : $53 \mathrm{~mm}$ - Age : 1 -

Premier circulus de l'année $1986(\triangleright)$.

Figure 26c: Chub scale (x 50).

Flume (Brittany).

Catch : 3.07.86.

FL : $53 \mathrm{~mm}$ - Age : $1+$.

First circulus of year $1986(\bullet)$. 


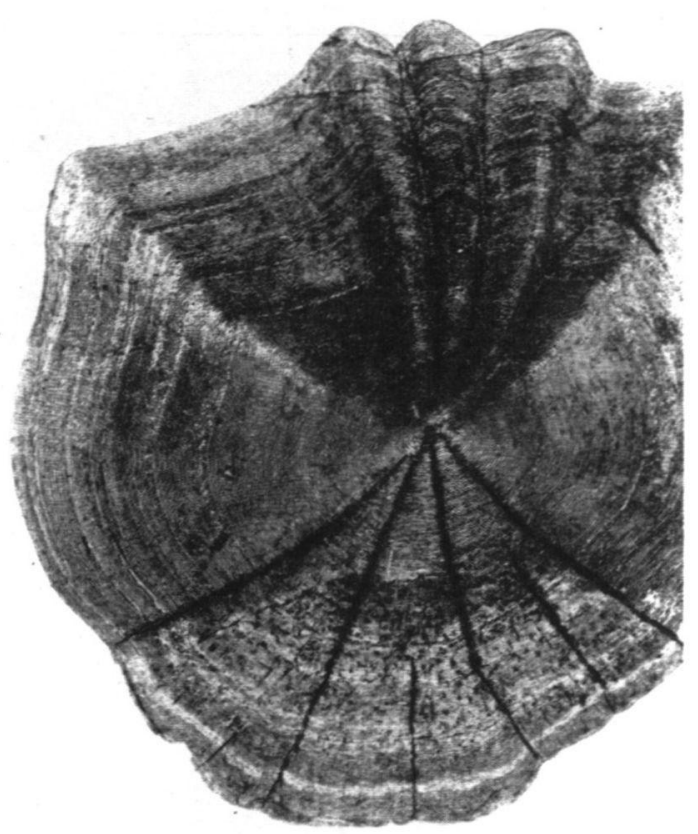

Figure 27a : Écaille de brème bordelière (x 10).

Retenue de St-Thurial (Bretagne).

Capture : 17.06.82

LF : $271 \mathrm{~mm}$ - $Q$ - Age : 6 +.

Figure 27a: Bream scale (x 10).

St Thurial reservoir (Brittany). Catch : 17.06.82.

FL : $271 \mathrm{~mm}$ - $q$ - Age : 6 t.

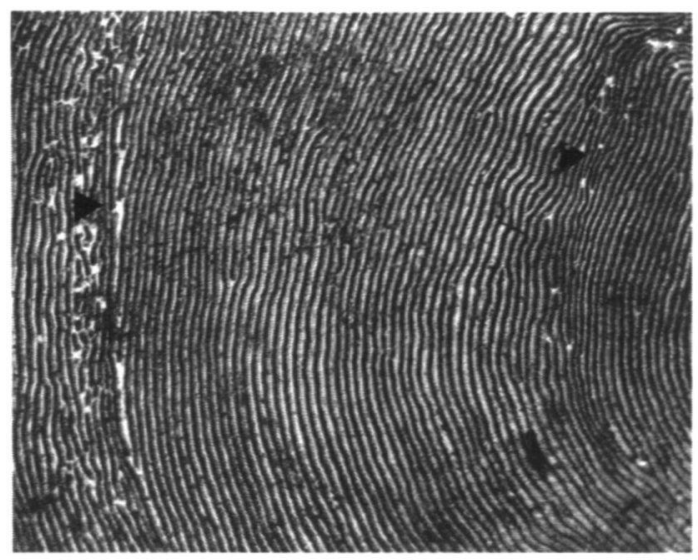

Figure 27b : Bandes hivernales ( $(\downarrow)$ sur une écaille de brème bordelière (x 50).

Figure 27b : Winter bands $(\downarrow)$ on bream scale (x 50).

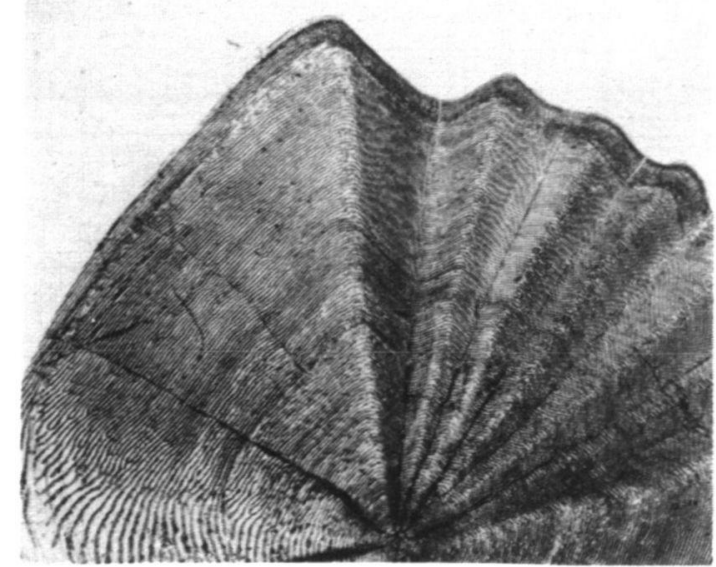

Figure 28a : Ecaille de poisson rouge (x 10). Etang de Parthenay (Bretagne) Capture : 2.04 .86

LF : $210 \mathrm{~mm}$ - $Q$ - Age : 3 l.

Figure 28a : Gold fish scale (x 10).

Parthenay Pond (Brittany).

Catch : 2.04.86.

FL : $210 \mathrm{~mm}$ - $Q$ - Age : 3 ।.

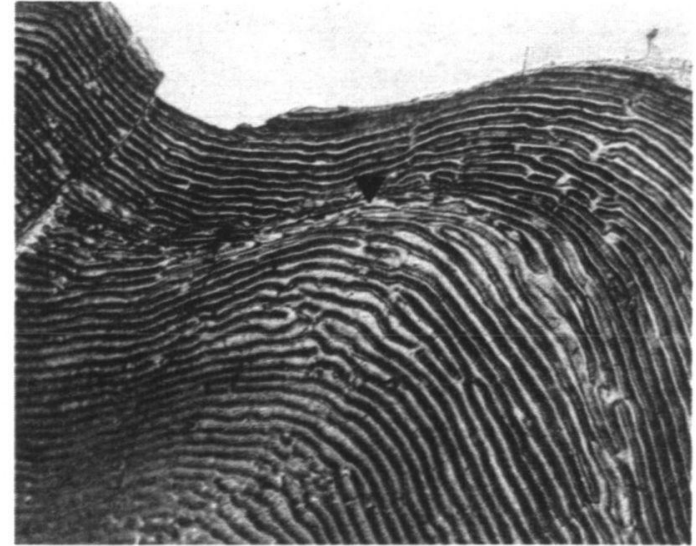

Figure 28b : Arrêt hivernal $(-)$ sur une écaille de poisson rouge $(x 50)$.

Figure 28b : Winter band $(\bullet)$ on gold fish scale (x 50). 


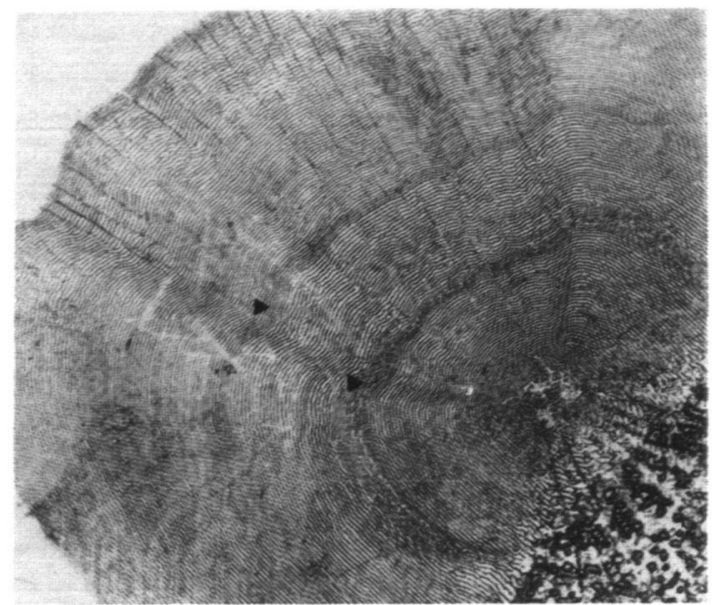

Figure 29 : Écaille de carpe (x 10).

Étang de Crévin (Bretagne).

Capture : 22.09.83.

LF : $247 \mathrm{~mm}$ - Age : 2 †.

Anneau hivernal .

Figure 29 : Carp scale (x 10).

Crevin Pond (Brittany).

Catch : 22.09.83.

FL : $247 \mathrm{~mm}$ - Age : 2 ł.

Winter band $\bullet$.

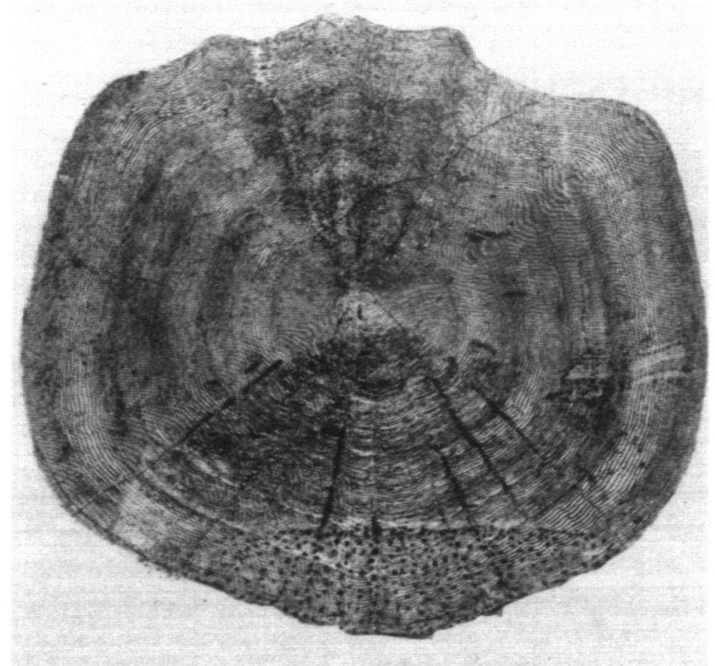

Figure 31a : Écaille de brème commune (x 10).

Lac de Guerlédan (Bretagne).

Capture : 13.05 .81 .

LF : $266 \mathrm{~mm}$ - Age : $4+$.

Figure 31a : Common bream scale (x 10). Guerledan Lake (Brittany).

Catch : 13.05.81.

FL : $266 \mathrm{~mm}$ - Age : $4+$.

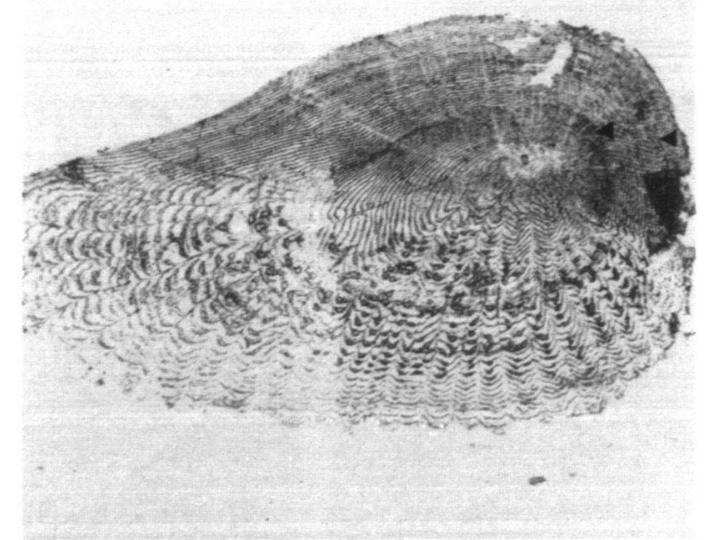

Figure 30 : Ecaille de carpe Miroir (x 10). La Flume (Bretagne).

Capture : 27.08.80.

LF : $160 \mathrm{~mm}$ - Age : 2 †.

Anneau hivernal $\bullet$.

Figure 30 : Mirror carp scale (x 10).

La Flume (Brittany).

Catch : 27.08.80.

FL : $160 \mathrm{~mm}$ - Age : $2+$.

Winter band

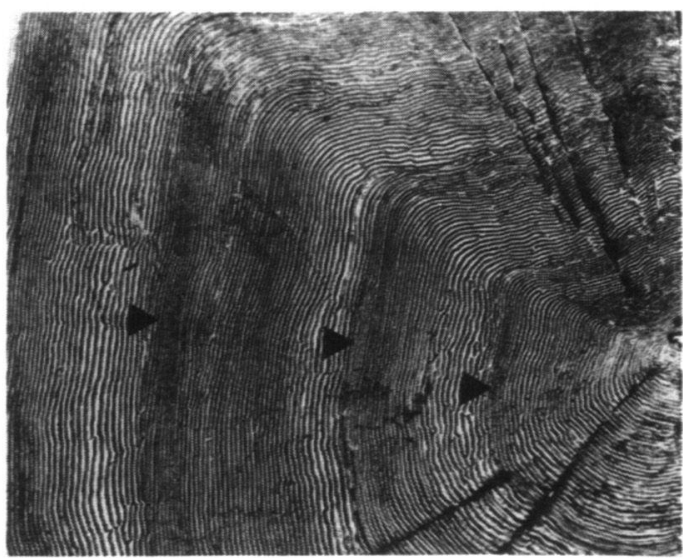

Figure 31b : Arrêts hivernaux ( $)$ sur une écaille de brème commune de $3+$ ans (x 30)

Figure $31 b$ : Winter bands $(\downarrow)$ on $3+$ years old common bream scale (x 30). 


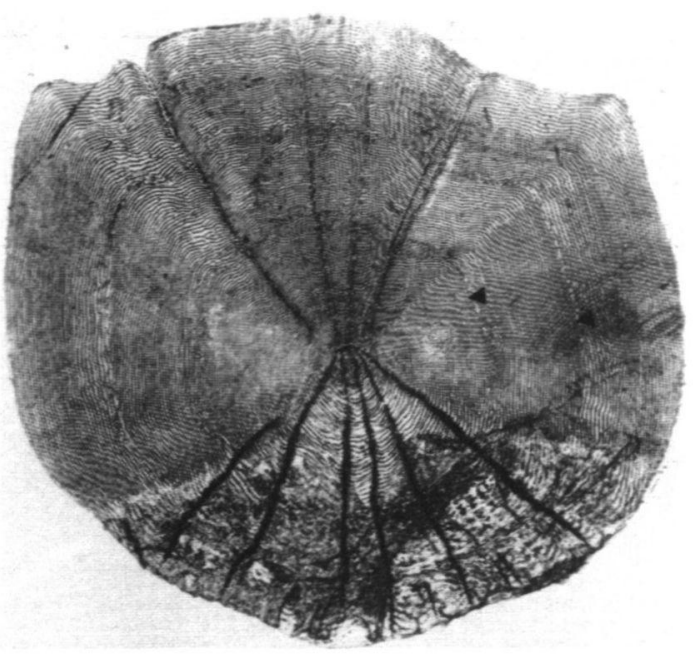

Figure 32 : Écaille de carassin (x 10). Étang de Pacé (Bretagne). Capture : 4.12.77. LF : 220 mm - Age : $2+$ Anneau hivernal $\bullet$.

Figure 32 : Crucian carp scale (x 10). Pacé Pond (Brittany).

Catch : 4.12.77.

FL : $220 \mathrm{~mm}$ - Age : $2+$. Winter band $\bullet$.

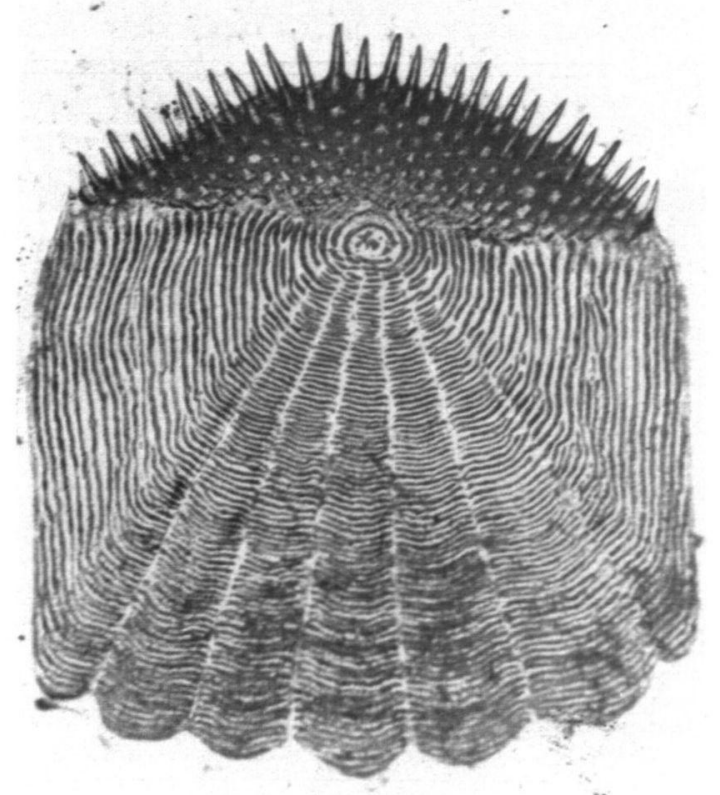

Figure 34a : Ecaille de sandre (x 20). Lac de Guerlédan (Bretagne). Capture : 13.05.81. LF : 325 mm - of Aye : 2+.

Figure 34a : Pike-perch scale (x 20). Guerledan Lake (Brittany). Catch : 13.05.81. FL : 325 mm - of - Age : 2- .

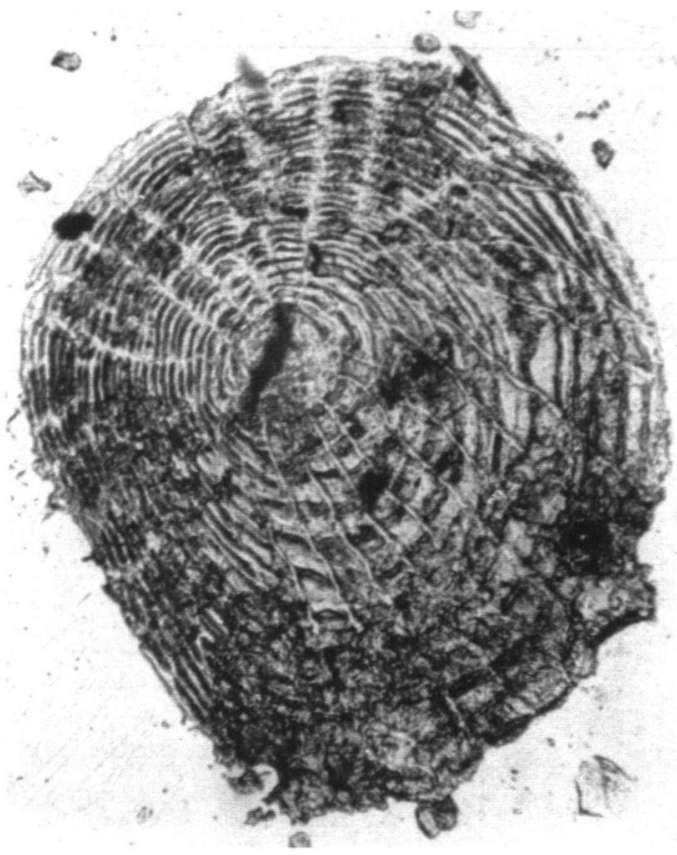

Figure 33 : Écaille de vairon (x 100). Colmont (Mayenne).

Capture : 4.10.80

LF : $67 \mathrm{~mm}$.

Age difficile à déterminer.

Figure 33 : Minnow scale (x 100).

Colmont (Mayenne).

Catch : 4.10.80.

FL : $67 \mathrm{~mm}$.

Difficulty for age determination.

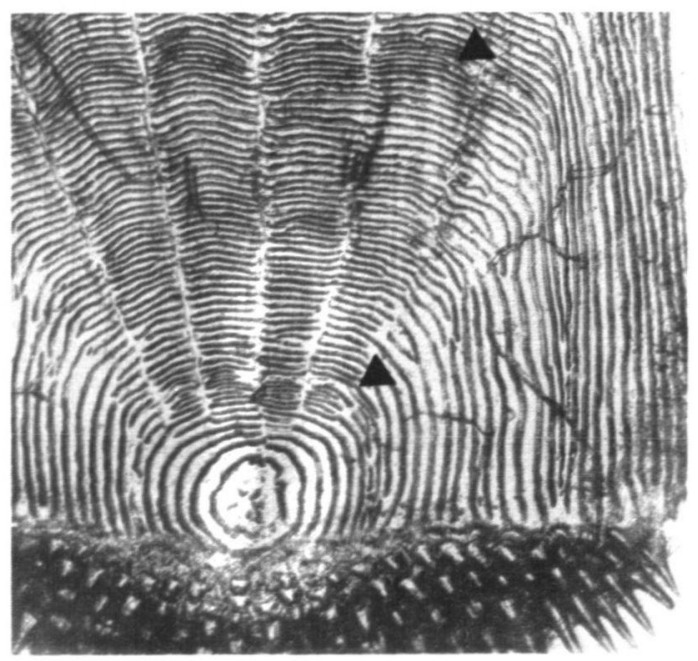

Figure 34b : Arrêts hivernaux $(\nabla)$ sur une écaille de sandre de $2+$ ans (x 50).

Figure 34b : Winter bands $(-)$ on $2+$ years old pike-perch scale $(\times 50)$. 


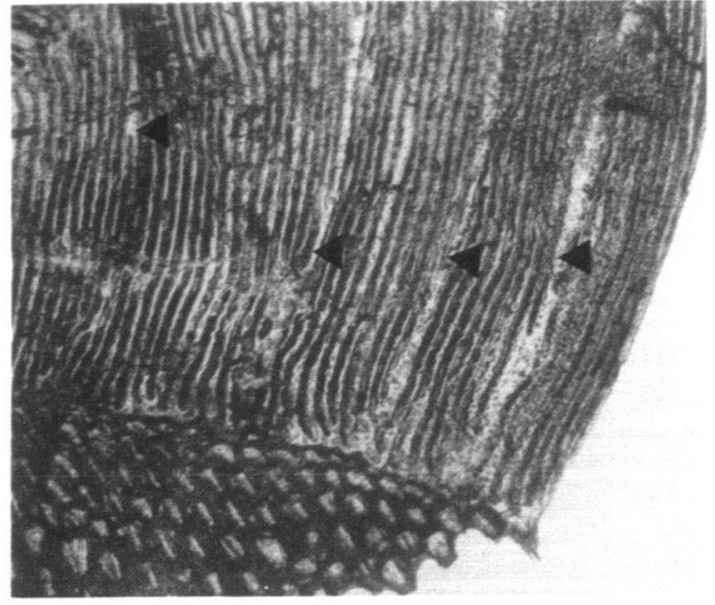

Figure 34c : Structure de bandes hivernales $(\mapsto)$ sur une écaille de sandre $(x$ 50)

Lac de Guerlédan (Bretagne).

Capture : 14.05.81.

LF : $633 \mathrm{~mm}-\delta$ - Age : 4 .

Figure 34c : Winter bands structure $(\bullet)$ on pike-perch scale (x 50).

Guerledan Lake (Brittany).

Catch : 14.05.81.

FL : $633 \mathrm{~mm}-\delta$ - Age : 4 t.

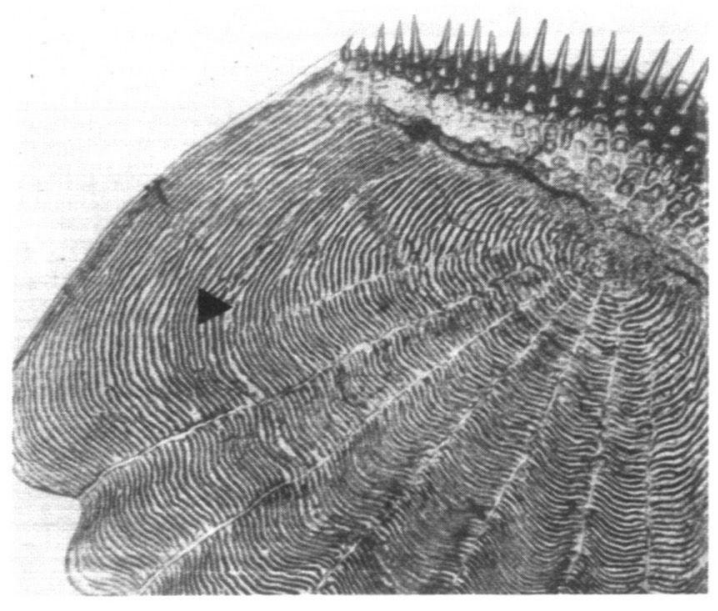

Figure 35b : Arrêt hivernal $(-)$ sur l'écaille d'une perche de $1+$ an (x 20).

Figure 35b : Winter band $(\bullet)$ on $1+$ year old perch scale $(\times 20)$.

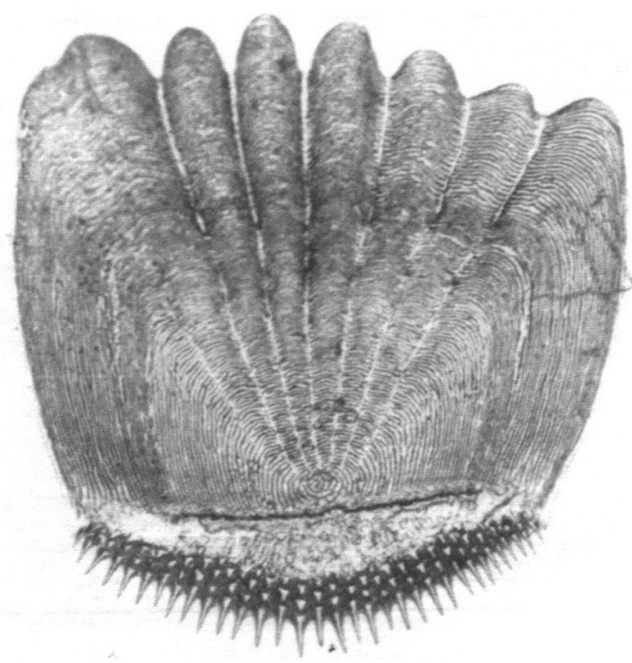

Figure 35a : Écaille de perche (x 10). Le Canut (Bretagne). Capture : 29.11.84. LF : 146 mm - $q$ - Age : 1 t.

Figure 35a: Perch scale (x 10). Le Canut (Brittany). Catch : 29.11.84.

FL : $146 \mathrm{~mm}$ - $Q$ - Age : $1+$.

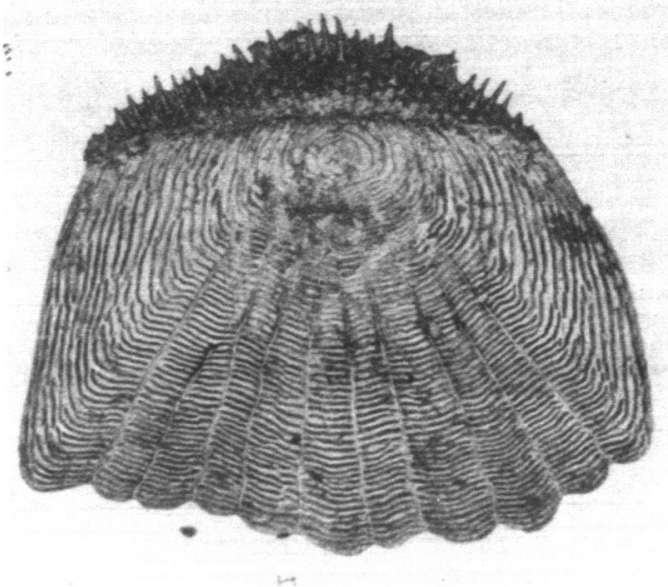

Figure 36a : Écaille de grémille (x 20). Le Meu (Bretagne). Capture : 9.11.84. LF : $118 \mathrm{~mm}$. Age difficile à déterminer: $1+$ ?

Figure 36a : Ruffe scale (x 20). Le Meu (Brittany). Catch : 09.11.84. FL : $118 \mathrm{~mm}$.

Difficulty for age determination : 1t? 


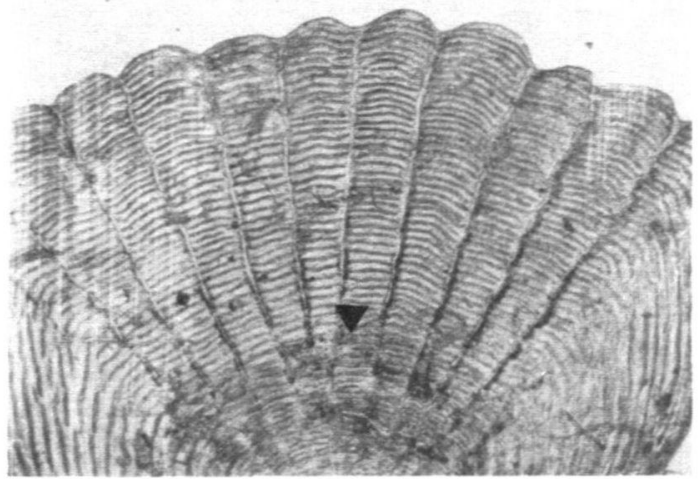

Figure 36b: Possible anneau hivernal ( $\bullet$ sur une écaille de grémille (x 50).

Figure 36b : Possible winter band $(t)$ on ruffe scale (x 50).

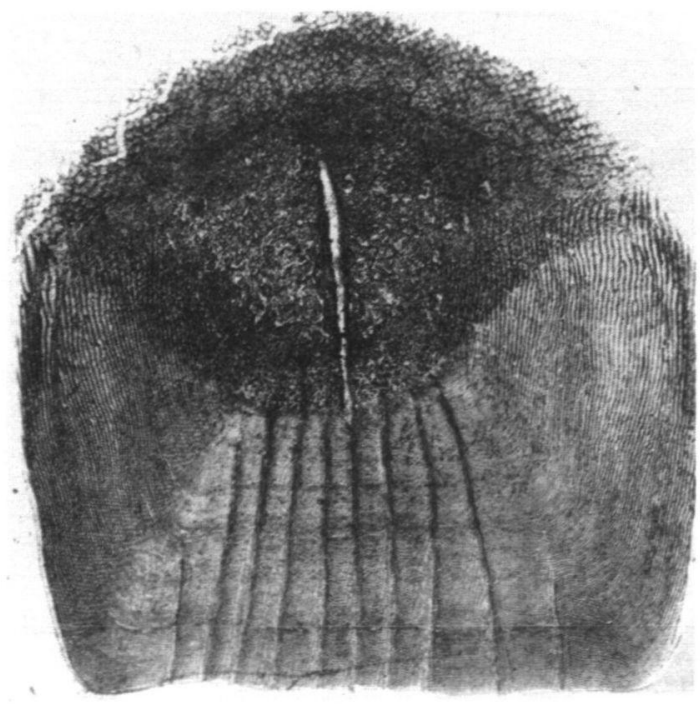

Figure $37 \mathrm{~b}$ : Écaille de mulet Chelon labrosus (x 10).

Côte atlantique (Bretagne).

Capture : 23.10.84.

LF : $318 \mathrm{~mm}$.

Age présumé : 3 †. 4 .

Figure 37b : Grey mullet Chelon labrosus scale (x 10).

Atlantic coast (Brittany).

Catch : 23.10.84.

FL : $318 \mathrm{~mm}$.

Possible age : $3+.4$.

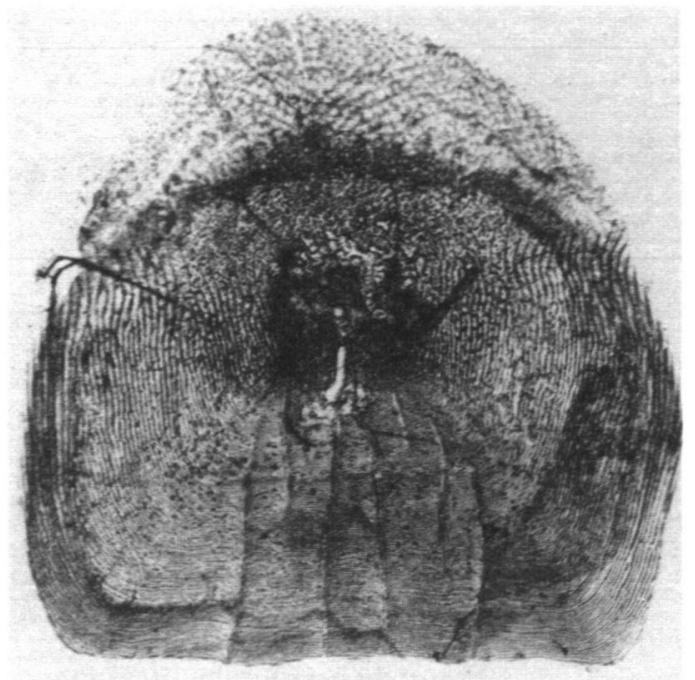

Figure 37a : Ecaille de mulet Liza auratus (x 10).

Côte atlantique (Bretagne).

Capture : 24.10.84.

LF : $270 \mathrm{~mm}$.

Age présumé : 2 †. 3.

Figure 37a : Grey mullet Liza auratus scale (x 10).

Atlantic coast (Brittany).

Catch : 24.10.84.

FL : $270 \mathrm{~mm}$.

Possible age : $2+.3$.

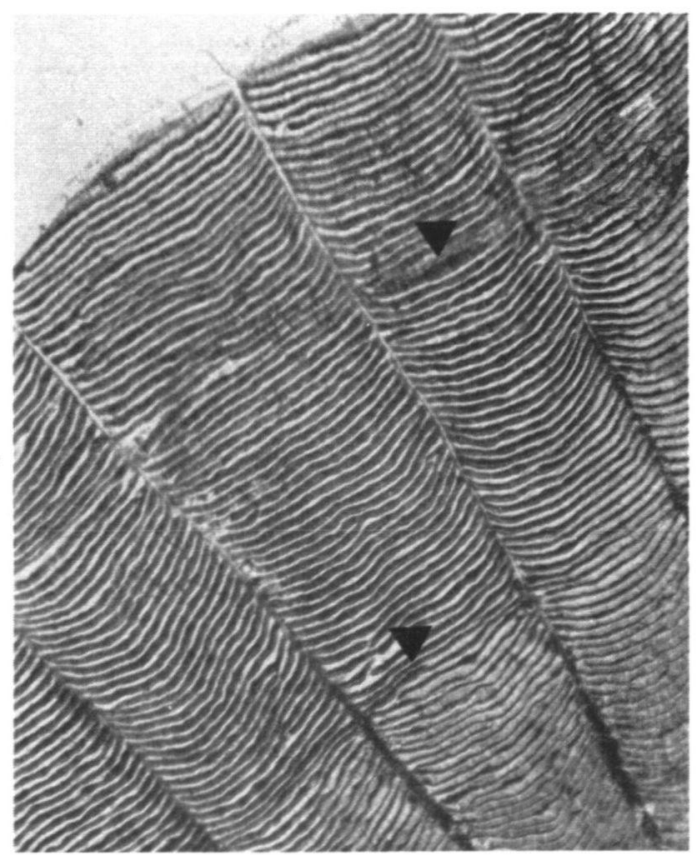

Figure 37c: Bandes hivernales $(\downarrow)$ sur une écaille de mulet (Liza aurafus) (x 50).

Figure 37c : Winter bands $(\downarrow)$ on grey mullet (Liza auratus) scale (x 50). 


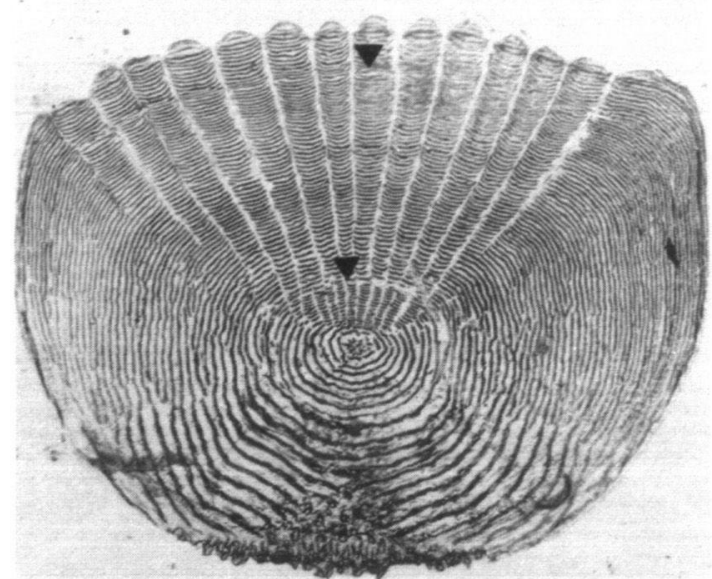

Figure 38 : Ecaille de perche soleil (x 30). Étang de la Guerche (Bretagne). Capture : 5.04.78.

LF : $114 \mathrm{~mm}$ - Age : $2+$ Bande hivernale $(\overrightarrow{)}$ ).

Figure 38 : Yellow perch scale (x 30). Guerche Pond (Brittany). Catch : 5. 04.78.

FL : $114 \mathrm{~mm}$ - Age : 2+. Winter band $(\bullet)$.

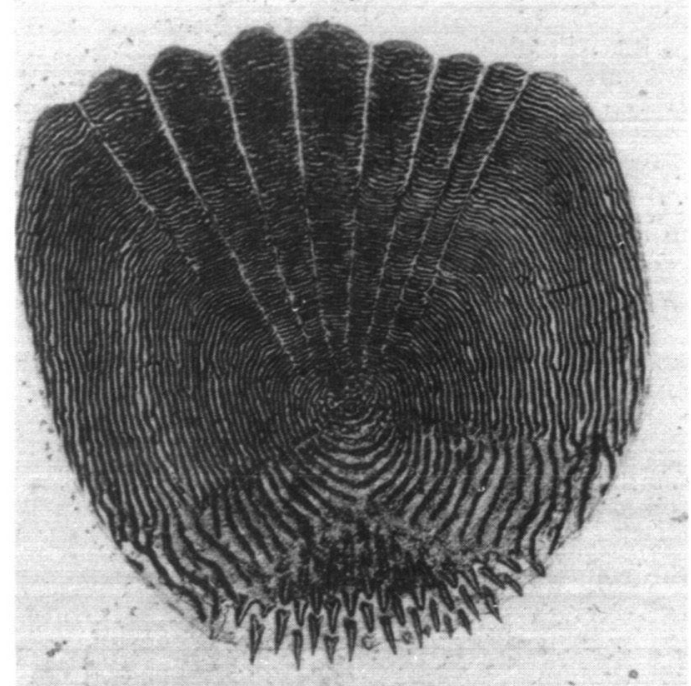

Figure 39a : Ecaille de black-bass (x 20). Seine (lle de France). Capture : 13.08 .74 . LF : $140 \mathrm{~mm}$. - Age : $1+$.

Figure 39a : Black bass scale (x 20). Seine (Ile de France). Catch : 13.08 .74 FL : $140 \mathrm{~mm}$ - Age : $1+$.

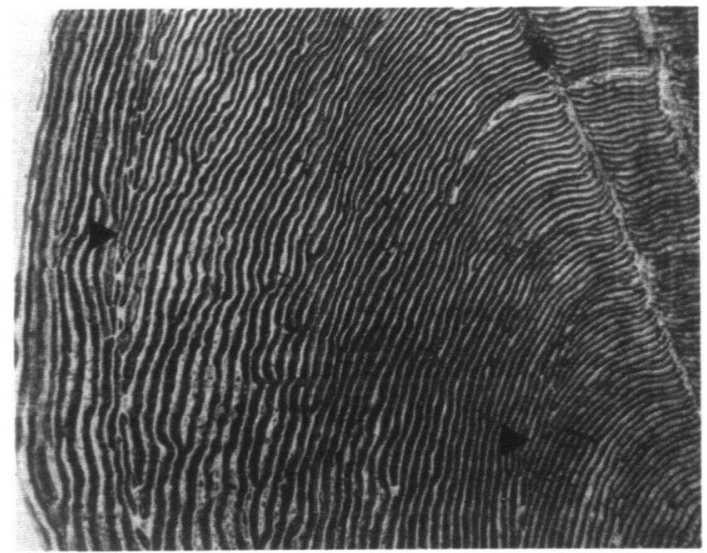

Figure 39b: Bandes hivernales $(\bullet)$ sur une écaille de black-bass (x 50). Age : $2+$.

Figure $39 \mathrm{~b}$ : Winter bands $(\omega)$ on black bass scale ( $x$ 50).

Age : $2+$. 


\section{DISCUSSION - CONCLUSION}

La forme et la structure de l'écaille peuvent évoluer au cours du temps (SIRE, 1986). Une véritable caractérisation des espèces entrainerait l'utilisation de techniques performantes telles que la microscopie à balayage électronique (SIRE et GERAUDIE, 1983; BURDAK, 1986), la microradiographie (SIRE et MEUNIER, 1981 ; MEUNIER, 1983), et la photographie stéréoscopique (BURDAK, 1986). Les études utilisant ces techniques permettent de connaitre l'évolution ontogénique et phylogénique de l'écaille (SIRE et GÉRAUDIE, 1983; MEUNIER, 1983; MEUNIER, 1984 b; BURDAK, 1986). Tel n'était pas l'objectif de cette étude. Le premier but était la reconnaissance des principales espèces de poissons dulçaquicoles rencontrées en France à partir d'une technique simple : la microscopie optique. L'identification se limitait à la phase adulte. II semble que dans ces conditions, l'objectif soit atteint.

Le deuxième but de ce travail était de synthétiser les données apportées par la méthode scalimétrique de la lecture d'âge et de les appliquer aux poissons étudiés. Dans cette optique, pour les espèces chez lesquelles la méthode scalimétrique ne peut être envisagée, une technique courante de détermination d'âge a été précisée. Si pour la quasi-totalité des poissons analysés la méthode scalimétrique reste fiable, elle apparaît vraiment comme un outil modèle et performant pour certaines espèces ou familles, notamment celle des salmonidés. Cependant, les limites d'applications de la scalimétrie dans certains cas nécessitent de plus en plus la mise en place d'études comparatives de la détermination de l'âge avec d'autres méthodes biologiques (BAGLINIÉRE et al., 1986: BOISNEAUMENNESSON et al., 1986) ou mathématiques (BOISNEAU-MENNESSON et al., loc. cit.) avec utilisation de systèmes de marquage (BOET, 1981 ; SIRE et MEUNIER, 1981; SIRE et GERAUDIE. 1983).

En dépit de ces réserves, la méthode de détermination de l'âge à partir des écailles reste d'utilisation simple dans les études d'Ecologie, d'autant qu'elle ne modifie pas le suivi d'une population dans son approche dynamique. Elle nécessite néanmoins une bonne connaissance du cycle biologique des espèces.

\section{REMERCIEMENTS}

Nous remercions Messieurs ALLARDI (CEMAGREF), BURGER (INRA), CARMIE (CSP ClermontFerrand), CHAMPIGNEULLE (INRA), FONTENELLE (ENSAR), MAISSE (INRA), PORCHER (CSP Rennes) et RICHARD (CSP Rennes) pour nous avoir fourni les écailles des espèces de poissons qui nous manquaient.

\section{BIBLIOGRAPHIE}

ALBERTINI-BERHAUT J., 1978. Croissance linéaire et pondérale de Mugil auratus comparée à celle de Mugil capito, Cybium, $4: 51-60$.

ALLARDI J., 1973. Connaissance de la biologie du black-bass (Micropterus salmoides) (Lacépède, 1802). DEA Paris VI, $20 \mathrm{pp}$

ALLARDI J., 1982. Introduction et acclimatation des poissons d'eau douce en France. EIFAC Iechn. Pap. 42 (2). 427-435.

ALLEN J.R.M., WOOTTON R.J. , 1982. Age, growth and rate of food consumption in an upland population of the three-spined stickleback, Gasterosteus aculeatus L. J. Fish Biol., 21 : 95-105.

ANONYME, 1984. Atlantic Salmon Scale Reading. ICES, Aberdeen 23-28 April, 15 pp.

BACKIEL T., SYCH R., 1958. Scales resorption and spawning marks in sea trout (Salmo trutta L.) from polish waters. Roc. Nauk Rol., 73 ser. B : 119-148.

BAGLINIÉRE J.L., 1985. La détermination de l'âge par scalimétrie chez le saumon atlantique/Salmo salar) dans son aire de répartition méridionale : utilisation pratique et difficultés de la méthode. Bull. Fr. Pêche Piscic., 298 : 69-105.

BAGLINIERE J.L., LECLERC C., RICHARD A., 1986. Comparaison entre l'âge et la croissance déterminés par scalimétrie et otolimétrie chez la truite de mer (Salmo trutta). Bull. Fr. Pêche Piscic., $301: 56-66$

BERNET B., 1960. Recherches biologiques sur les populations de Gobio gobio de la Nivelle. Ann. Stat. Cent. Hydro. Appl. $8: 127-180$.

BERRA T., 1981 A Allas of distribution of freshwater fish Families of the World. Ed. Univ. Nebraska Press, 1 vol., 197 pp.

BILTON H.T., ROBINS G.L., 1971a. Effects of feeding level on circulus formation on scales of young sockeye salmon (Oncorhynchus nerka). J. Fish Res. Bd. Canada, 28 : $861-868$.

BILTON H.T., ROBINS G.L., $1971 \mathrm{~b}$. Effects of starvation, feeding and light period on circulus formation on scales of young sockeye salmon (Oncortiunchus nerka). J. Fish. Res. Bd. Canada, 28:17491755 . 
BILTON H.T., ROBINS G.L., 1971c. Response of young sockeye salmon (Oncorhynchus nerka) to prolonged period of starvation, J. Fish. Res. Bd. Canada, $28: 1757-1761$.

BILTON H.T., 1974. Effects of starvation and feeding on circulus formation on scales of young sockeye salmon of four racial orıgıns, and one race of young kokanee, coho and chinook salmon : 40-70 in Bagenal T.B. Ed., The ageing of fish. Unwin Brothers Ltd., London.

BOET P., 1981. Éléments d'Écologie du poısson chat, (/ctalurus melas (Rafinesque, 1820)), du Lac de Créteil. Thèse de Docteur de $3^{e}$ cycle, Fac. Sci Univ. Paris VI, 1 vol., 123 pp.

BOET P., LE LOUARN H., 1985. La croissance du poisson. Techniques d'étude : in GERDEAUX D. et BILLARD R. Ed., Gestion piscicole des lacs et retenues artificielles. INRA Paris, 125-142.

BOISNEAU-MENNESSON C., BOISNEAU Ph., BAGLINIĖRE J.L., 1986. Premières observations sur les caractéristiques biologiques des adultes de grande alose (Alosa alosa L.) dans le cours moyen de la Loire. Acta Oecologica Oecol Apptic. 7, 337-353

BURDAK V.D. 1986. Morphologie fonctionnelle du tégument écailleux des poissons. Cybium, 10 (n० 3 supplément), 1 vol., $147 \mathrm{pp}$.

CASSELMANN J.M., 1967. Age and growth of Northern pike (Esox /ucius Linnaeus) of the Upper St Lawrence River. M.S. University of Guelph, 219 p.

CASSELMANN J.M. 1974. Analysis of hard tissue of Pike (Esox /ucius) with special reference to age and growth. 13-27. in T.B. Bagenal Ed., The ageing of fish. Unwin Brother's Ltdt., London.

CASSOU-LEINS F. CASSOU-LEINS J.J., 1981. Recherche sur la biologie et I'halieutique des migrateurs de la Garonne et principalement de l'alose. (Alosa alosa Linné). Thèse $3^{\text {e }}$ cycle, Inst. Nat. Polytechn. Toulouse, $392 \mathrm{pp}$.

CERVIA J.L., TORRES S., 1983. On the growth and reproduction of two populations of gudgeon (Gobio gobio L.) in central Spain. Acta Hydrobiol., 25-26: 101-115.

CHUGUNOVAN.I., 1963. Age and growth studies in fish. Irans. Nat. Sci. Found. Washington, 132 pp.

CRISP D.T., 1963. A preliminary survey of brown trout (Salmo trutta L.) and bullheads (Cottus gobio L.) in high altitude becks. Salm. Trout. Mag., 167:45-59.

CUERRIER J.P., 1951. The use of pectoral fin rays for determining age of sturgeon and other species of fish. Can. Fish. Cult., $11: 10-18$

DAHL K.. 1909. The assessment of age and growth in fish. Int. Revue der gesamt. Hydrobiol. und Hydrogr., $2: 758-769$

DEMPSON J.B., GREEN J.M., 1985. Life history of anadromous arctic charr, (Salvelinus alpinus), in the Fraser River, Northern Labrador. Can J. Zool., $63: 315-324$.

DENIEL C., 1981. Les poissons plats (Téléostéens - Pleuronectiformes) en Baie de Douarnenez. Thèse de Docteur ès Sciences, Fac. Sci. Techn. Univ. Bresı, 1 vol., 476 pp.

DE PONTUAL H., 1986. Discrimination des stocks de saumon atlantique (Salmo salar L.) par analyse de la forme de leurs écailles. Thèse $3^{\text {e }}$ cycle. Fac. Sci. Univ. Paris VI (sous presse).

DOUCHEMENT C., 198i. Les aloses des fleuves français : Alosa fallax Lacépède et Alosa alosa Linné. Biométrie. Ecobiologie. Autonomie des populations. Thèse $3^{e}$ cycle. Écol. gen. app. Montpellier I, $393 \mathrm{pp}$.

DUBUIT M.H., BEAUPOIL C., 1985. Les poissons marins de Bretagne Laboratoire de biologie marine du Collège de France, Concarneau, $24 \mathrm{p}$.

DUMAS J., 1976. Dynamique et sédentarité d unc population naturalisée de truite arc-en-ciel (Salmo gairdneri Richardson) dans un ruisseau de montagne, l'Estibère (Hautes Pyrénées). Ann. Hydrobiol., $7: 117 \cdot 139$.

FRENETTE J.F., DODSON J.J., 1984. Brook Trout /Salvefinus fontinalis/ population structure in acidified Lac Tantaré, Québec. Can J. Fish. Aquat. Sci. 41 : 865-877.

GAJDUSEK J., 1981. Growth, age and mortality of bream (Abramis brama), in the Mostiste Water Reservoir. Folia. Zool., $30: 215-228$.

GERDEAUX D., 1986. Écologie du gardon (Rutilus rutilus L.) et du sandre (Lucioperca lucioperca L.) dans le lac de Créteil de 1977 à 1982 Etude de la ligulose du gardon. Thèse de Docteur ès Sciences, Fac. Sci., Univ. Paris VI, 1 vol., 161 pp.

GRASSE P.P., 1958. Traité de zoologie. Tome XIII. 1er fascicule. Masson Ed. Paris, 924 p.

GULLESTAD N., 1974. On lack of winter zones in the centre of scales from Arctic Char (Salmo alpinus L.) Norw. J. Zool., 22 : $141-143$.

HARTLEY P.H.T., 1947. The natural history of some British freshwater fishes. Proc. Zool. Soc. Lond., $117: 129-206$

HELLAWELL J.M., 1969. Age determination and growth of the grayling (Thymallus thymallus (L.)) of the River Lugg, Herefordshire. J. Fish. Biol., 1 : 373-382. 
HESTHAGEN T., 1985. Validity of the age determination from scales of brown trout (Salmo trutta L.) Int. Freshw. Res. Drottingholm Rep., $62: 65-70$.

HOFFBAUER C., 1898. Die Altersbestimmung des Karpfens an seiner Shuppe. All. Fish. Zeitung., $23: 341-343$.

HOFSTEDE A.E., 1974. Studies on growth, ageing and back-calculation of roach (Rutilus rutilus (L.)) and dace (Leuciscus leuciscus (L.)) : in Bagenal T.B., Ed., The ageing of fish. Unwin Brothers Ltd. London.

HOGMAN W.J., 1968. Annulus formation on scales of four species of Coregonids reared under artificial conditions. J. Fish. Res. Board Canada, $25: 2111-2122$.

JARVI T.H., MENZIES N.J.M., 1936. The interpretation of the zones on scales of salmon, sea trout and brown tout. Cons. Perm. Expl. Mer XCVII, 1 vol. 63 pp.

JEARLD A., 1983. Age determination. In : Fisheries techniques 301 -324. Nielsen L. Ed. Blacksburg.

JONES J.W., HYNES H.B.N., 1950. The age and growth of Gasterosteus aculeatus, Pygosteus pungitius and Spinachia vulgaris. as shown by their otoliths. J. Anim. Ecol., 19 : 59-73.

JONSSON B., 1976. Comparaison of scales and otoliths for age determination in brown Trout (Salmo trutta L.) Norv. J. Zool., 24 : 295-301.

JONSSON B., OSTLI T., 1979. Demographic strategy in char compared with brown trout in Lake Lone, Western Norway. Inst. Freshw. Drottningholm. $58: 45-54$

KIRKA A. 1969. Age composition, growth, maturity and fecundity of Salmo trutta morpha fario Linnaeus, 1758 and Cottus poecilopus Heckel, 1836 in the Drava and Vah River Basins. Prace LR, $2: 185-218$.

KOZIKOWSKA S., 1976. Les corégones, leur biologie et les possibilités d'augmenter leur production. Rev. Trav. Inst. Pêches marit., 40 (3 et 4), 644-645.

KRAIEM M., 1979. Ecologie du barbeau fluviatile, (Barbus barbus (L. 1758)) (Poissons, Cyprinidés) dans le Haut-Rhône Français. Thèse $3^{e}$ cycle Biol. Anim. Zool. Fac. Sci., Univ. Lyon 1, 1 vol., 75 pp.

LAM HOAI THONG, 1969. Contribution à l'étude de la biologie des mugilidés (poissons téléostéens) des côtes du Massif armoricain. Trav. Fac. Sci. Rennes Ser. Océanogr. Biol., 2 : 55-182.

LEA E., 1910. On the methods used in herring investigations Publ. Circ. Cons. Perm. Int. Explor. Mer no 53

LE CREN E.D., 1947. The determination of age and growth of the perch (Perca fluviatilis) from the opercular bone. J. Anim. Ecol., $16: 188-204$.

LIBOSVARSKY J., 1956. Wachsen des Döbels und Revision der zwei Hauptmethoden seiner Rückbestimmung. Zool. listy, 5 : 302-314.

LIBOSVARSKY J., 1976. Lepidological note on grey mullet (Mugil capito) from Egypt. Zool. listy. $25: 73-79$.

LIEDER U., 1969. Uber Jahresmarken und Störungzonen auf dem Schuppen der Teichcypriniden. Z. Fisherei, $8: 67-86$

LINDFIELD R.S., 1974. The errors likely in ageing roach (Rutilus rutilus (L.)) with special reference to stunded populations : in Bagenal T.B. Ed., The ageing of fish, Unwin Brothers Ltd., London.

MACK A.. BILLARD R., 1984. Données bibliographiques sur l'élevage des jeunes stades et la biologie des Corégones. Bull. Fr. Piscic., 292 : $18-45$.

MAISSE G., BAGLINIĖRE J.L., 1983. Mise en évidence d'une marque de fraie dans la phase eau douce des écailles de saumon atlantique du Massif armoricain. ICES CM 1983/M : $28: 17$.

MAITLAND P.S., 1972. Key to British freshwater fishes. Freshwater Biological Association. Scientific publication $\mathrm{n}^{\circ} 27,139 \mathrm{p}$.

MAISSE G., BAGLINIĖRE J.L., LEBAIL P.Y., 1987. Dynamique de population de truite commune (Salmo trutta) d'un ruisseau breton (France): les géniteurs sédentaires. Hydrobiologia, 148 : 123-130.

MANN R.H.K., STEINMETZ B., 1985. On the accuraty of age-determination using scales from rudd (Scardinius ervthrophthalmus (L.)) of known age. J. Fish. Biol., 26 : 621-628.

MARZOLF R.C., 1950. Use of pectoral spines and vertebrae for determining age and rate of growth of the channel fish. $J$. Wildl. Manage., $19: 243-249$.

MELHAOUI M., 1985. Eléments d'écologie de la truite de Lac (Salmo trutta L.) du Léman dans le système Lac-affluent. Thèse de $3^{e}$ cycle. Fac. Sci. Univ. Paris VI, 1 vol., 127 pp.

MENON M.D., 1950. The use of bones other than otoliths in determining the age and growth rates of fishes. J. Const. Perm. Int. Explor. Mer., $16: 311-340$. 
MEUNIER F.J., 1983. Les tissus osseux des Osteichtyens. Structure, genèse, croissance et évolution. Thèse doctorat d'état, Sciences, Paris, Archives et documents, Microédition, Inst. Ethnol. Mus. Nat. Hist. Nat., SN 82-600-328, 200 pp.

MEUNIER F.J., 1984a. Structure et minéralisation des écailles de quelques Osteoglossidae (Osteichtyens, Teleosteens). Ann. Sci. Nat., Zool., 13e série, 6:11-124

MEUNIER F.J., 1984b. Spatial Organization and Mineralisation of the basal Plate of Elasmoid scales in Osteichthyans. Amer. Zool, $24: 953-964$.

MEUNIER F.J., PASCAL. M., 1980. Quelques données comparatives sur la croissance périodique des tissus squelettiques chez les Osteichthyens. Bull. Soc. Zool. Fr., 105 : 337-342.

MICHA J.C., 1971. Densité de population, âge et croissance du barbeau (Barbus barbus (L.)) et de l'ombre (Thymallus thymallus (L.)) dans l'Ourthe. Ann. Hydrobiol, 2 : 45-68.

MILLS C.A., WELTON J.S., RENDLE E.L., 1983. The age, growth and reproduction of the stone loach (Noemacheilus barbatulus (L.)) in a Dorset chalk stream. Fresh. Biol., 13 : 283-292.

MONGEAU J.R., 1960. Croissance du brochet commun, (Esox lucius L.), dans deux lacs du Parc du Mont Tremblant, province de Québec. Thèse de Doctorat ès Sciences (Biologie). Université de Montréal, $150 \mathrm{pp}$.

NELVA-PASQUAL A., 1985. Biogéographie, Démographie et Ecologie de Chondrostoma nasus nasus L., (1758) (Hotu, Poisson, Téléostéen, Cyprinide). Thèse d'État UER Sciences de la Nature, Fac. Sci., Univ. Lyon 1, 1 vol. 349 pp

NESBIT R.A., 1934. A convenient method for preparing celluloid impressions of fish scales. J. Cons. Perm. Int. Explor. Mer, $9:$ 373-376.

OTTAWAY E.M., SIMKISS K., 1977. A method for assessing factors influencing "false check" formation in fish scales. J. Fish. Biol., $11: 681-687$.

OTIAWAY E.M., 1978. Rhythmic growth activity in fish scales. J. Fish. Biol., $12: 615-623$.

PERSAT H. 1977. Ecologie de l'ombre commun. Rapport final $2^{\mathrm{e}}$ édition 1978. ARALEP BP Univ. Lyon I. $84 \mathrm{pp}$

PETERSON R.H., SUTTERLIN A.M., METCALFE J.L., 1979. Temperature preference of several species of Salmo and Salvelinus and some of their hybrids. J. Fish. Res. Board Canada. 39:1137-1140.

PETROSKY C.E., WATERS T.F., 1975. Annual production by the slimy sculpin population in a small Minnesota trout stream. Trans. Am. Fish. Soc., 104: 237-244.

PHILIPPART J.C., 1971. Age et croissance de la vandoise (Leuciscus leuciscus L.) dans I'Ourthe et la Berwine. Ann. Soc. Royale Zool. de Belgique, 101: 77-140.

PHILIPPART J.C., 1972. Age et croissance du chevaine (Leuciscus cephalus L.) dans l'Ourthe et la Berwine. Ann. Soc. Rovale de Belgique, 102:47-81

PROKES M. LIBOSVARSKY J., BARUS V., 1977. Scale growth in juvenile chub from the Rokytna stream. Fol. Zool., $26: 277-288$

PROTOPOPOV N.K., 1980. The formation of spawning rings and supplementary rings on the scales of the Pechora River whitefish, (Coregonus lavaretus pidshian). J. Ichthyol., 20 : 38-48.

QUIGNARD J.P., AUTHEM M., 1981. Structure des populations de Liza ramada (Poisson, Mugilidés) des côtes languedociennes de Carnon au Rhône. Age et croissance de ce poisson. Cybium. $5: 49-63$.

RICHARD A., 1986. Les populations de truite de mer, (Salmo trutta L.), des rivières Orne et Touques (Basse Normandie): Scalimétrie. Sexage. Caractéristiques biométriques el démographıques. Thèse $3^{\mathrm{e}}$ cycle. Fac. Sci. Univ. Rennes, $54 \mathrm{p}$.

RICHARD M., 1973. Croissance et densité de popuiation du chevaine (Leuciscus cephalus L.) dans la Seine au niveau de Montereau (Seine et Marne). Thèse de $3^{e}$ cycle. Paris VII, $62 \mathrm{pp}$

SAEED D.W. LIBOSVARSKY J., 1983. Scale growth in roach (Rutilus rutilus) from the Musov Reservoir, Czechoslovakia. Fol. Zool. $32: 375 \cdot 382$.

SIRE J.Y., 1981. La scaliation (apparition et mise en place des écailles) chez Hemichromis bimaculatus (GILL, 1862) (Téléostéens, Perciformes, Cichlidés) Cybium. $5: 51-66$.

SIRE J.Y., 1986 Ontogenic development of surface ornementation in the scales of Hemichromis bimaculatus (Cichlidae). J. Fish. Biol., 28:713-724.

SIRE J.Y. MEUNIER F.J., 1981. Structure et minéralisation de l'écaille d'Hemichromis bimacu/atus (Téléosten, Perciforme. Cichlidé). Arch. Zool. Gén. Exp., 122 : 133-150

SIRE J.Y., GERAUDIE J., 1983. Fine structure of the developping Scale in the Cichlid (Hemichromis bimaculatus) (Pisces, Teleostei, Perciformes). Acta Zoologica, $64: 1$-8

SMILY W.J.P., 1955. On the biology of the stone loach (Noemacheilus barbatula (L.)) Ann. Ecol., 24. 167-186. 
TROUVERY M., WILLIOT P., 1984. Biologie et Ecologie d'Acipenser Sturio. Etude la Pêcherie. I- Biologie et Ecologie d'Acipenser sturio. CEMAGREF Division ALA Etude n०17: 1-42.

VAN OOSTEN J., 1957. The skın and scales, 207-244 in Brown M.E. Ed. The physiology of fishes, Vol. 1 : Metabolism. Acad Piess. Inc., New York.

WALLIN O., 1958. On the growth, structure and developmental physiology of the scale of fishes, Rep. Inst. Freshw. Res. Drottningholm, $38: 385-447$

WILLIAMS J.E., 1955. Determination of age from the scales of northern pike (Esox lucius L.) Ph. D University of Michigan, 185 pp.

WILLIAMS W.P., 1967. The growth and mortality of four species of fish in the River Thames at Reading. J. Anim. Ecol., 36 : 695-720.

WURTZ-ARLET J., 1952. Le black-bass en France. Esquisse monographique Ann. Stat. Centr. Hydrol. App/. IV, 1-84. 


\author{
$2^{e}$ annonce \\ COLLOQUE SUR LA TRUITE COMMUNE \\ (SALMO TRUTTA L.) \\ CONSEIL SUPÉRIEUR DE LA PÉCHE \\ Centre du Paraclet \\ (articles et commentaires en français) \\ 6-7.8 Septembre 1988 \\ organisé par
}

\author{
2nd announcement \\ CONFERENCE ON BROWN TROUT \\ (SALMO TRUTTA L.) \\ CONSEIL SUPERIEUR DE LA PĖCHE \\ Centre du Paraclet \\ (articles and commentaries in french) \\ 6-7-8 September 1988 \\ organized by
}

CONSEIL SUPÉRIEUR DE LA PẼCHE Bulletin Français de la Pèche et de la Pisciculture :

Gestion des Ressources Aquatiques
INSTITUT NATIONAL DE LA RECHERCHE AGRONOMIQUE

Station de Physiologie et d'Écologie des Poissons

\section{COMITÉ D'ORGANISATION}

J.L. BAGLINIĖRE, J.M. JANTZEN, G. MAISSE, E. VIGNEUX

\section{COMITÉ SCIENTIFIQUE}

J. ALLARDI, J. ARRIGNON, J.L. BAGLINIÉRE, A. CHAMPIGNEULLE, B. CHEVASSUS, Y. COTE, P. DUMONT, G EUZENAT, Françoise FOURNEL, M. HELAND, J.C. LEFEUVRE, G. MAISSE, A. NEVEU A. NIHOUARN, J.C. PEDROLI, J.C. PHILIPPART, A. RICHARD, E. VIGNEUX.

\section{PROGRAMME - PROGRAMME}

La truite (Salmo Trutta L.) de rivière, de lac et de mer; Écologie et gestion des populations dans le milieu naturel.

The trout (Salmo Trutta L.), the brown, lake and sea one; Ecology and populations management in natural environment.

\section{I - BIOLOGIE DES POPULATIONS SAUVAGES BIOLOGY OF WILD POPULATIONS}

Caractéristiques des populations, dynamique des stocks, stratégies démographiques. Populations characteristics, dynamic of stocks, demographic strategies.

II - Place dans l'écosystéme

Place in the ecosystem

Habitat, facteurs trophiques, compétition, reproduction..

Habitat, trophic factors, competition, reproduction...

III - GESTION DES POPULATIONS DANS LE MILIEU NATUREL Populations management in natural environment

Aménagement, repeuplement et exploitation...

Amenagement, restockement and exploitation...

IV - TECHNIQUES D'ÉTUDE Technical studies

Dénombrement, marquage, détermination d'âge, sexage.

Census, tagging. age determination, sexing. 